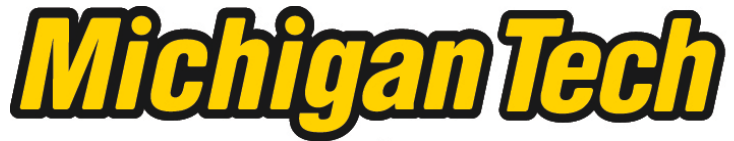 \\ Michigan Technological University Create the Future Digital Commons @ Michigan Tech
}

2011

\section{Multi-antenna non-line-of-sight identification techniques for target localization in mobile ad-hoc networks}

Wenjie Xu

Michigan Technological University

Follow this and additional works at: https://digitalcommons.mtu.edu/etds

Part of the Electrical and Computer Engineering Commons

Copyright 2011 Wenjie Xu

\section{Recommended Citation}

$\mathrm{Xu}$, Wenjie, "Multi-antenna non-line-of-sight identification techniques for target localization in mobile adhoc networks", Dissertation, Michigan Technological University, 2011.

https://doi.org/10.37099/mtu.dc.etds/58

Follow this and additional works at: https://digitalcommons.mtu.edu/etds

Part of the Electrical and Computer Engineering Commons 
MULTI-ANTENNA NON-LINE-OF-SIGHT IDENTIFICATION TECHNIQUES FOR

TARGET LOCALIZATION IN MOBILE AD-HOC NETWORKS

\author{
By
}

Wenjie Xu

\begin{abstract}
A DISSERTATION
Submitted in partial fulfillment of the requirements for the degree of DOCTOR OF PHILOSOPHY

(Electrical Engineering)
\end{abstract}

MICHIGAN TECHNOLOGICAL UNIVERSITY

2011

(C) 2011 Wenjie Xu 

This dissertation, "Multi-Antenna Non-Line-Of-Sight Identification Techniques for Target Localization in Mobile Ad-hoc Networks," is hereby approved in partial fulfillment of the requirements for the degree of DOCTOR OF PHILOSOPHY IN THE FIELD OF ELECTRICAL ENGINEERING.

Department of Electrical and Computer Engineering

Signatures:

Dissertation Advisor

Dr. Seyed A. (Reza) Zekavat

Committee Member

Dr. Daniel R. Fuhrmann

Committee Member

Dr. Zhi (Gerry) Tian

Committee Member

Dr. Vladimir D. Tonchev

Department Chair

Dr. Daniel R. Fuhrmann

Date 



\section{Contents}

List of Figures $\ldots \ldots \ldots \ldots \ldots \ldots \ldots \ldots \ldots \ldots \ldots \ldots$

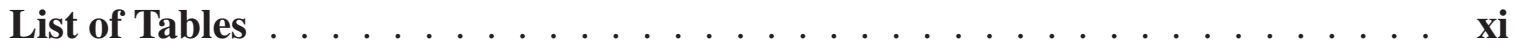

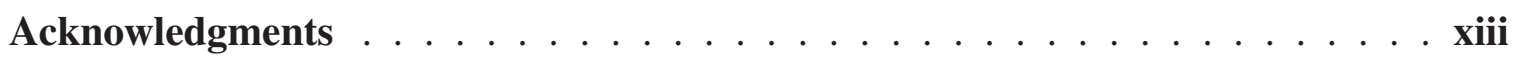

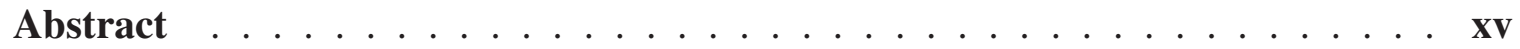

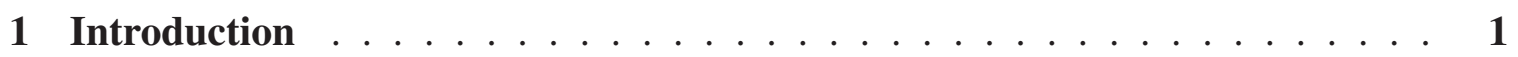

1.1 Motivation ...................... 2

1.2 NLOS identification: a statistical detection problem . . . . . . . . 4

1.3 Different categories of NLOS identification techniques $\ldots \ldots \ldots$

1.4 The main idea $\ldots \ldots \ldots \ldots \ldots \ldots \ldots \ldots \ldots \ldots$

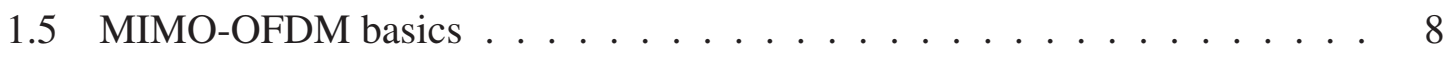

$1.5 .1 \quad$ OFDM basics $\ldots \ldots \ldots \ldots \ldots \ldots \ldots$

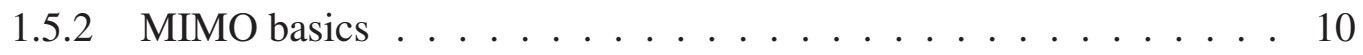

1.6 Chapter contributions . . . . . . . . . . . . . . . 12

2 A Review on NLOS Identification Techniques in the Literature . . . . . . 15 


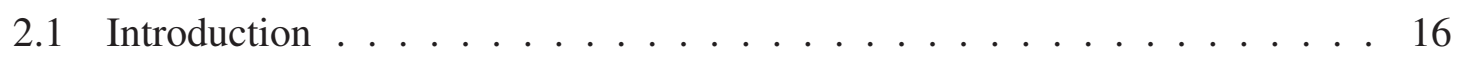

2.2 Cooperative NLOS identification . . . . . . . . . . . . 17

2.2 .1 DOA residual testing $\ldots \ldots \ldots \ldots \ldots$

2.2.2 Time-difference-of-arrival (TDOA) residual . . . . . . . . . 18

2.2.3 Residual distribution testing . . . . . . . . . . . . . . . . 19

2.3 NLOS identification based on the range statistics $\ldots \ldots \ldots \ldots . \ldots 21$

2.3.1 Techniques based on range measurements over time . . . . . . 22

2.3.2 Techniques based on the range measurements over different frequency bands $\ldots \ldots \ldots \ldots \ldots \ldots \ldots \ldots$

2.4 NLOS identification based on channel characteristics . . . . . . . . . 27

2.4.1 Narrow and wideband systems $\ldots \ldots \ldots \ldots \ldots$

2.4.2 Ultra-wide-band (UWB) systems . . . . . . . . . . . . . . . . . . . 29

2.5 Hybrid approaches $\ldots \ldots \ldots \ldots \ldots \ldots \ldots \ldots$

2.6 Comparison of NLOS identification methods . . . . . . . . . . . 36

2.7 Conclusion $\ldots \ldots \ldots \ldots \ldots \ldots \ldots \ldots \ldots \ldots \ldots \ldots \ldots$

3 NLOS Identification Using Spatial Correlation $\ldots \ldots \ldots$

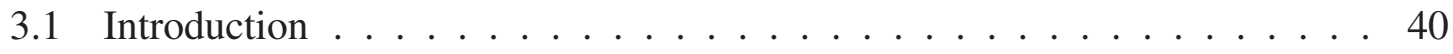

3.2 multipath two-user MIMO channel matrix $\ldots \ldots \ldots \ldots$

3.2.1 NLOS and LOS channel matrices . . . . . . . . . . . . . . 44

3.2.2 Comparison with other channel models . . . . . . . . . . . . 49

3.3 Statistics of the scattered field $\ldots \ldots \ldots \ldots \ldots \ldots \ldots \ldots \ldots$ 
3.4 Numerical results and analysis . . . . . . . . . . . . . 66

3.5 Conclusion . . . . . . . . . . . . . . . 72

\section{NLOS Identification via Phase Difference Statistics across Two Antenna El-}

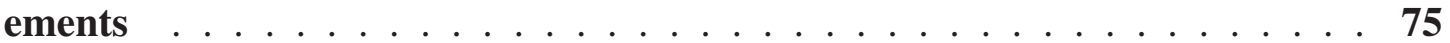

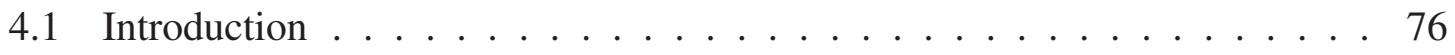

4.2 Received signal model and $K$-factor estimator . . . . . . . . . . . . . . . 77

4.3 NLOS identification based on estimated Rician $K$-factor . . . . . . . . . . . 84

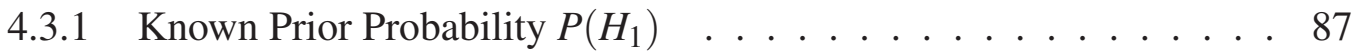

4.3.2 Unknown Prior Probability $P\left(H_{1}\right) \ldots \ldots$. . . . . . . . . . . . 89

4.4 Uncorrelated phase verification . . . . . . . . . . . . . . 90

4.5 Simulation results . . . . . . . . . . . . . . . . . 96

4.6 Conclusion . . . . . . . . . . . . . . . 100

\section{NLOS Identification in Frequency Selective and Space Selective radio chan-}

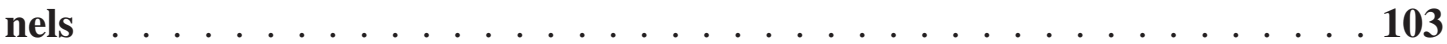

5.1 Introduction . . . . . . . . . . . . . . . . . 104

5.2 Techniques based on space-frequency channel correlation . . . . . . . . 105

5.2.1 Space-frequency channel correlation . . . . . . . . . . . 105

5.2.2 Definition of subcarrier correlation difference . . . . . . . . . 108

5.2.3 Characteristics of SCD and SCD statistics across antenna pairs . . . 112

5.3 Numerical simulations and discussions . . . . . . . . . . . . . . . . . 114

5.3.1 Absolute value of SCD . . . . . . . . . . . . . . 116 
5.3.2 Mean and variance of SCD . . . . . . . . . . . 116

5.4 Conclusion . . . . . . . . . . . . . . . . . . 119

6 NLOS Identification in Time-Varying, Frequency Selective and Space Selective radio channels $\ldots \ldots \ldots \ldots$. . . . . . . . . . . . . . . . . . . .

6.1 Introduction . . . . . . . . . . . . . . . . . . 122

6.2 Space-time-frequency channel model of MIMO-OFDM systems . . . . . 123

6.3 Proposed measures: $\Lambda(N / 2), \Lambda(0)$ and $\Omega \ldots \ldots$. . . . . . . . . 126

6.4 Numerical simulations and discussions . . . . . . . . . . . . . . . 129

6.5 Conclusion . . . . . . . . . . . . . . . . . . 134

7 Conclusions and Open problems $\ldots \ldots \ldots$

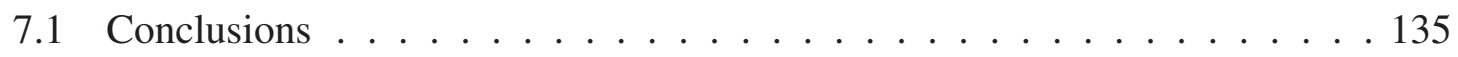

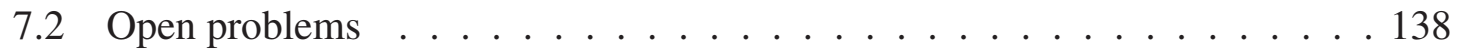

7.2.1 Practical issues in employing spatial correlation for NLOS identifi-

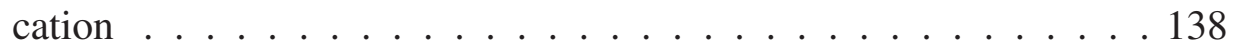

7.2.2 In Chapter 4, what if $\operatorname{cov}\left(\Delta \phi_{1} \Delta \phi_{2}\right) \neq 0 \ldots . . \ldots . \ldots 139$

7.2.3 Space-time tap correlation model . . . . . . . . . . . . 139

7.2.4 Measures based on multi-dimensional space-time tap correlation . . 140

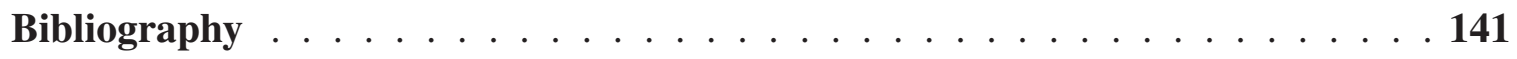

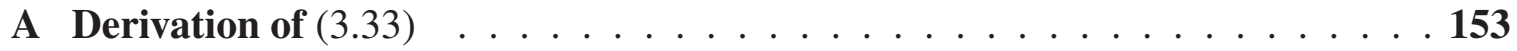




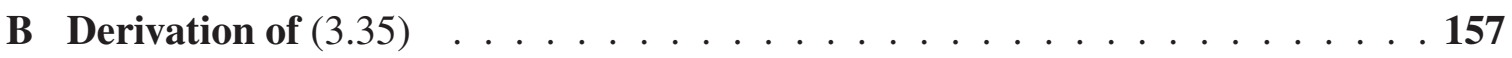

C Probability density function of $\Delta \phi_{1} \ldots \ldots \ldots \ldots$ 


\section{List of Figures}

1.1 Localization error in NLOS propagation environment. . . . . . . . . . . 3

1.2 A big picture of NLOS identification . . . . . . . . . . . . . . 4

1.3 Channel correlation across time . . . . . . . . . . . . . 6

1.4 (a) Channel correlation across frequency (b) Channel correlation across dis-

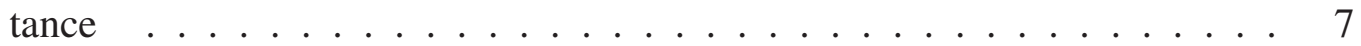

1.5 Multicarrier transmitter . . . . . . . . . . . . . . . . . 9

1.6 OFDM with IFFT/FFT implementation . . . . . . . . . . . . . . 10

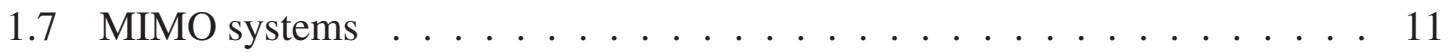

2.1 NLOS identification based on DOA residual testing . . . . . . . . . 17

2.2 The relationship of DOD and DOA of LOS component . . . . . . . . 36

3.1 Two-user channel for the scattering environment within a valley. . . . . . . 43

3.2 Signal arrivals and departures for multi-user MIMO channel. . . . . . . . . 44

3.3 The scattering geometry. . . . . . . . . . . . . . 53

3.4 Statistics of the scattering coefficients: $\mathrm{E}\left(\rho \rho^{*}\right)$ and $\mathrm{E}\left(\rho\left(\rho^{\prime}\right)^{*}\right)$ for $T=25 \lambda$, $L=250 \lambda, \theta_{1}=\theta_{2}=45^{\circ} \ldots \ldots \ldots \ldots \ldots$ 
3.5 Statistics of the scattering coefficients: $\mathrm{E}\left(\rho \rho^{*}\right)$ and $\mathrm{E}\left(\rho\left(\rho^{\prime}\right)^{*}\right)$ for $T=25 \lambda$, $L=250 \lambda, \theta_{1}=\theta_{2}=5.625^{\circ} \ldots \ldots \ldots \ldots \ldots \ldots \ldots \ldots \ldots \ldots \ldots \ldots$

3.6 Statistics of the scattering coefficients: $\mathrm{E}\left(\rho \rho^{*}\right)$ and $\mathrm{E}\left(\rho\left(\rho^{\prime}\right)^{*}\right)$ for $T=25 \lambda$, $L=250 \lambda, \theta_{1}=45^{\circ}, \theta_{2}=22.5^{\circ} \ldots \ldots \ldots \ldots$

3.7 Illustration of beam oscillation technique. . . . . . . . . . . 63

3.8 Simulation set-up. . . . . . . . . . . . . . . 66

3.9 Comparison of LOS and NLOS spatial cross-user correlation for $\sigma=0.5 \lambda, 10 \lambda$ when $\mathrm{D}(\mathrm{BS}, \mathrm{User} 1)=3000 \lambda \ldots \ldots \ldots$

3.10 Comparison of LOS and NLOS spatial cross-user correlation for $\sigma=0.5 \lambda, 10 \lambda$ when $\mathrm{D}(\mathrm{BS}, \mathrm{User} 1)=10000 \lambda \ldots \ldots \ldots$

3.11 Comparison of LOS and NLOS spatial cross-user correlation along $\mathrm{X}$ axis $(\mathrm{Y}$ coordinate $=0)$ for $\sigma=0.5 \lambda, 10 \lambda$ when $\mathrm{D}(\mathrm{BS}$, User 1$)=3000 \lambda, 10000 \lambda .70$

3.12 Comparison of LOS and NLOS spatial intra-user correlation versus BS's antenna spacing for $\sigma=0.5 \lambda, 10 \lambda$ when $K=1,4 \ldots \ldots$. . . . . 71

3.13 LOS and NLOS spatial intra-user correlation for $\sigma=0.5 \lambda, 10 \lambda$ when User 1's antenna array is parallel to the surfaces. . . . . . . . . . . . . 72

4.1 Two-antenna receiver. . . . . . . . . . . . . . . 78

4.2 Received signals in vector space. . . . . . . . . . . . . . 79

4.3 PDFs of $\Delta \phi_{1}, I_{[-\pi, \pi]}\left(\phi_{\mathrm{LOS} 1}+\Delta \phi_{1}\right)$ and $I_{[0,2 \pi]}\left(\phi_{\mathrm{LOS} 1}+\Delta \phi_{1}\right)$ under the case $\phi_{\mathrm{LOS} 1} \approx \pi \ldots \ldots \ldots \ldots \ldots \ldots$

4.4 Polynomial least square fitting of $g(K) \ldots \ldots \ldots$. . . . . . . 83 
4.5 Block diagram of NLOS identification technique based on phase difference across two antenna elements . . . . . . . . . . . . . . . . . . 89

4.6 Geometrical configuration of (a) circular scattering (b) elliptic scattering. . . 91

4.7 Circular model: $\operatorname{coeff}\left(\Delta \phi_{1}, \Delta \phi_{2}\right)$ and coeff $\left(r_{\mathrm{DIF} 1}, r_{\mathrm{DIF} 2}\right)$ versus antenna spacing for (a) $\Delta=19.2^{\circ}$. (b) $\Delta=60^{\circ} \ldots \ldots \ldots$. . . . . . . . . 94

4.8 Circular model: comparison of simulated and theoretical $\sigma_{\Delta \phi}^{2}$ for (a) $\Delta=$ $19.2^{\circ}$. (b) $\Delta=60^{\circ} \ldots \ldots \ldots \ldots$

4.9 Elliptic model: (a) phase and signal correlation coefficients versus antenna spacing (b) comparison of simulated and theoretical $\sigma_{\Delta \phi}^{2} \ldots \ldots . . . . .95$

4.10 Performance comparison of $\hat{K}_{\Delta \phi}$ and $\hat{K}_{2,4}$ estimators with a sample size of $N=$ 500: (a) Root mean square error (b) Bias. . . . . . . . . . . . 96

4.11 For $\sigma_{\mathrm{sh}}=4 \mathrm{~dB}, 6 \mathrm{~dB}, 8 \mathrm{~dB}(\mathrm{a})$ PDF of $K_{\mathrm{dB}}^{\mathrm{NLOS}}$ and $K_{\mathrm{dB}}^{\mathrm{LOS}}$ (b) probability-ofdetection $P_{\mathrm{D}}$ V.S. probability-of-false alarm $P_{\mathrm{F}} \ldots \ldots \ldots$. . . . . . . 98

5.1 Geometry of scatterer distribution for mircocell environments . . . . . . . 109

$5.2 \chi_{l}^{p_{1}, q_{1} ; p_{2}, q_{2}}$ in micro cell environment . . . . . . . . . . . 111

5.3 The distributions of $\Gamma(N / 2)$ under LOS and NLOS scenarios when $p 1-$ $p 2=-1, q_{1}-q_{2}=-1$, Rician $K=1,4,10 \ldots \ldots \ldots \ldots$

5.4 The distributions of (a) $\mu$ and $\sigma$ (b) $\mu^{\text {abs }}$ and $\sigma^{\text {abs }}$ when Rician $K=1$. . 116

5.5 The distributions of (a) $\mu$ and $\sigma$ (b) $\mu^{\text {abs }}$ and $\sigma^{\text {abs }}$ when Rician $K=10 \ldots 118$

6.1 (a) Cluster scattering model A: $R_{a_{l}}$ is independent of $R_{a_{m}}, l \neq m$. (b) Cluster scattering model B, $R_{a_{l}} \approx R_{a_{m}}, l \neq m . \ldots \ldots 130$ 
6.2 The distributions of $\Lambda(N / 2)$ and $\Omega$ when Rician $K=0.5$ for cluster scattering model $\mathrm{A} \ldots \ldots . \ldots \ldots$. . . . . . . . . . . . . . . . . . . . . . . . .

6.3 The distributions of $\Lambda(N / 2), \Lambda(0)$ and $\Omega$ when Rician $K=0.5$ for cluster scattering model B ....................... 133 


\section{List of Tables}

2.1 A summary on range statistics based methods . . . . . . . . . . . . . 27

2.2 A summary on channel characteristics based methods . . . . . . . . . . . 34

2.3 Comparison of NLOS identification methods . . . . . . . . . . . . . 37

3.1 Comparison of four MIMO channel models. . . . . . . . . . . . . . 53

4.1 Comparison of $K_{\mathrm{th}}, P_{\mathrm{F}}$ and $P_{\mathrm{D}}$ for various $\sigma_{\mathrm{sh}} \ldots \ldots \ldots \ldots$

5.1 Comparison of decision threshold, $P_{F}$ and $P_{D}$ for various metrics when Rician $K=1 \ldots \ldots \ldots \ldots$. . . . . . . . . . . . . . . . . . . . 118

6.1 Comparison of $P_{F}$ and $P_{D}$ for $\Lambda(N / 2)$ and $\Omega$ when Rician $K=0.5,1,1.5$, 3 for cluster scattering model A . . . . . . . . . . . . . . 132

6.2 Comparison of $P_{F}$ and $P_{D}$ for $\Lambda(N / 2), \Lambda(0)$ and $\Omega$ when Rician $K=0.5$, $1,1.5,3$ for cluster scattering model B . . . . . . . . . . . 133 


\section{Acknowledgments}

I would like to express my gratitude to my advisor, Prof. Seyed Alireza (Reza) Zekavat. I thank him for his support, guidance and encouragement during the development of this work. Prof. Zekavat has been a key person for directing my research. He has played a great role in nurturing me professionally, especially in scientific writing.

It is my pleasure for having Prof. Daniel R. Fuhrmann, Prof. Zhi (Gerry) Tian and Prof. Vladimir D. Tonchev in my Ph.D. committee. Their comments and feedback throughout my proposal defense and final defense have been crucial to improve the quality of my dissertation.

Thank you to my colleagues in Michigan technological university, Xiaofeng Yang, Michael Roddewig, Andrew Kolbus, Stuti Agarwal, Jie Tang, Chao Li, Greg Price, Shu G. Ting, Dr. Mohsen Pourkhaataoun, Dr. Xiukui Li, especially Dr. Zhonghai Wang, Dr. Hui Tong for your close discussions and clarifications.

Thank you to my husband Daw Don Cheam for your life long committed love.

Thank you to my parents, Mr. Xu Xianru and Mrs Chen Lanzhu for your love, support and my very existence. Thank you to my parents-in-law, Mr. Cheam Soon Seong and Mrs Ow Choy Tsin and all other family members for your continuing support and love. 
Thank you to the Houghton Chinese Christ Disciple Church members, the Bethany Baptist Church members, the Pelkie Mennonite Church members, Mr. Glen Moilanen and Mrs Bonnie Moilanen for help, fellowship and encouragement.

Last but not least, thank you to my Lord Jesus Christ for shining in my life. 


\begin{abstract}
Target localization has a wide range of military and civilian applications in wireless mobile networks. Examples include battle-field surveillance, emergency 911 (E911), traffic alert, habitat monitoring, resource allocation, routing, and disaster mitigation. Basic localization techniques include time-of-arrival (TOA), direction-of-arrival (DOA) and received-signal strength (RSS) estimation. Techniques that are proposed based on TOA and DOA are very sensitive to the availability of Line-of-sight (LOS) which is the direct path between the transmitter and the receiver. If LOS is not available, TOA and DOA estimation errors create a large localization error. In order to reduce NLOS localization error, NLOS identification, mitigation, and localization techniques have been proposed.
\end{abstract}

This research investigates NLOS identification for multiple antennas radio systems. The techniques proposed in the literature mainly use one antenna element to enable NLOS identification. When a single antenna is utilized, limited features of the wireless channel can be exploited to identify NLOS situations. However, in DOA-based wireless localization systems, multiple antenna elements are available. In addition, multiple antenna technology has been adopted in many widely used wireless systems such as wireless LAN 802.11n and WiMAX 802.16e which are good candidates for localization based services.

In this work, the potential of spatial channel information for high performance NLOS identification is investigated. Considering narrowband multiple antenna wireless systems, two 
NLOS identification techniques are proposed. Here, the implementation of spatial correlation of channel coefficients across antenna elements as a metric for NLOS identification is proposed. In order to obtain the spatial correlation, a new multi-input multi-output (MIMO) channel model based on rough surface theory is proposed. This model can be used to compute the spatial correlation between the antenna pair separated by any distance.

In addition, a new NLOS identification technique that exploits the statistics of phase difference across two antenna elements is proposed. This technique assumes the phases received across two antenna elements are uncorrelated. This assumption is validated based on the well-known circular and elliptic scattering models. Next, it is proved that the channel Rician $K$-factor is a function of the phase difference variance. Exploiting Rician $K$-factor, techniques to identify NLOS scenarios are proposed.

Considering wideband multiple antenna wireless systems which use MIMO-orthogonal frequency division multiplexing (OFDM) signaling, space-time-frequency channel correlation is exploited to attain NLOS identification in time-varying, frequency-selective and spaceselective radio channels. Novel NLOS identification measures based on space, time and frequency channel correlation are proposed and their performances are evaluated. These measures represent a better NLOS identification performance compared to those that only use space, time or frequency. 


\section{Chapter 1}

\section{Introduction}

Systems capable of positioning mobiles remotely in wireless environments have emerging applications in homeland security, law enforcement, defense command and control, emergency services, and traffic alert, situation awareness, spacecraft orbit control, multirobot coordination, and vehicle-to-vehicle and vehicle-to-pedestrian collision avoidance. Many cooperative positioning methods based on direction-of-arrival (DOA), time-of-arrival (TOA), time-difference-of-arrival (TDOA), and received signal strength indication (RSSI) have been proposed. A common assumption across all these techniques is the availability of line-of-sight (LOS). In Non-LOS (NLOS) situations, the performance of the proposed methods highly decreases. Research is on-going to identify NLOS scenarios, reduce the localization error due to NLOS scenarios, and localize targets available in NLOS. NLOS identification avoids deceived positioning by reducing the effect of nodes that receive localization information through NLOS. NLOS identification techniques proposed in the lit- 
erature incorporate the availability of a single antenna, and mainly function based on the statistics of signal amplitude obtained over a single carrier frequency component. Today, many multi-antenna and multi-frequency radios and standards such as IEEE 802.11n that use multi-input multi-output (MIMO) orthogonal frequency division multiplexing (OFDM) systems have been introduced. This dissertation proposes novel NLOS identification methods that incorporate the capabilities of MIMO and OFDM systems to maintain high performance NLOS identification.

\subsection{Motivation}

Target localization has a wide range of military and civilian applications in wireless mobile networks. Examples include battlefield command and control [1], fire fighters tracking [2], emergency 911 (E911) [3], road traffic alert [4], resource allocation in mobile ad-hoc networks [5] and routing in sensor networks [6, 7] and etc.

The location of a target can be estimated by different parameters of the received radio signal, such as time-of-arrival (TOA) [8], direction-of-arrival (DOA) [9], time-differenceof-arrival (TDOA) [10] and received signal strength indication (RSSI) [11]. All these techniques require that the received signal travels through the line-of-sight (LOS) path which is the direct path between transceivers*. If the LOS path cannot be detected, i.e., in non-lineof-sight (NLOS) propagation conditions, the received signal will travel longer distances * Some RSSI based methods perform in NLOS scenarios; however, with a poor performance. 


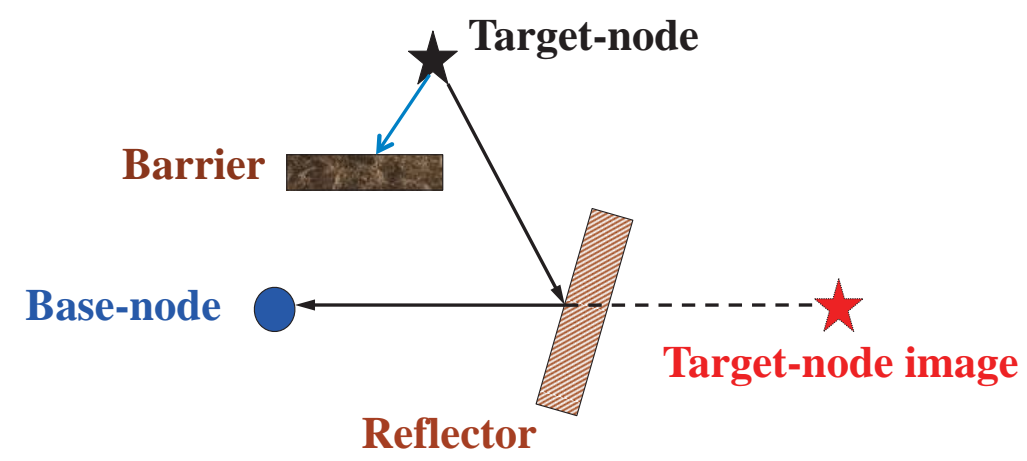

Figure 1.1: Localization error in NLOS propagation environment.

compared to the LOS path, which results in a larger TOA. In addition, in NLOS conditions, a wrong DOA estimation is expected (see Figure 1.1). Because of error in both DOA and TOA estimation, a large localization error is experienced. Thus, lack of availability of LOS has been known as the major source of localization errors. One method of reducing the NLOS localization error is to identify NLOS conditions. Identification results are used to mitigate the NLOS localization error [12].

Besides localization application, some NLOS identification methods offer LOS link quality information. Shown in [13], based on this information more complex TOA estimators can be selected for low quality links, i.e., the LOS path is detected but it is not the strongest in the multi-path profile. and less complex TOA estimators shall be selected for high quality links, i.e., the LOS path is detected and it is the strongest. In addition, identifying LOS conditions allows optimal adjustment of the transmission mode of communication systems by switching to a higher order of modulation for LOS links to achieve higher data rates. 


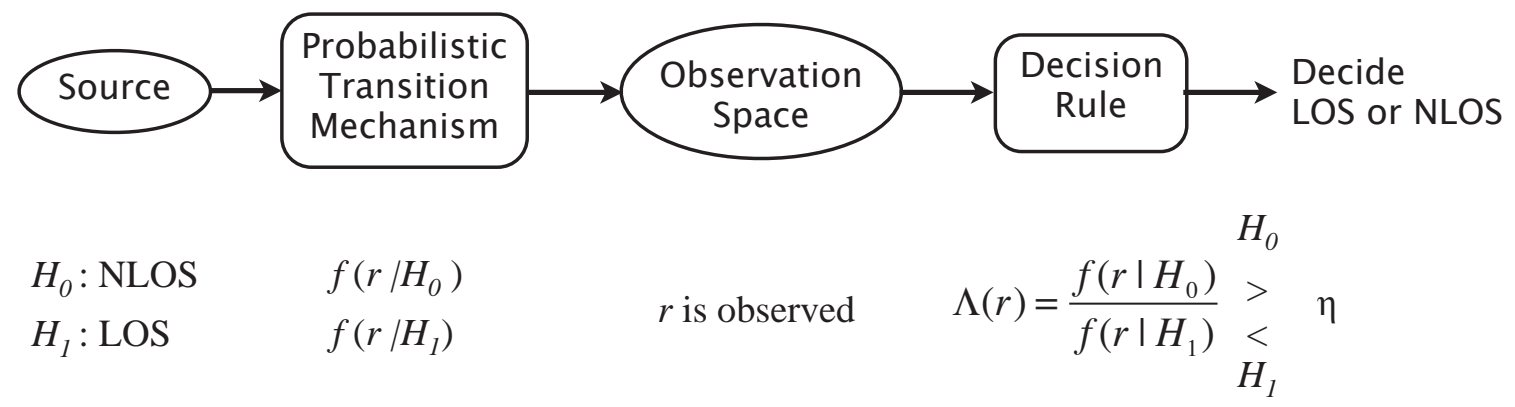

Figure 1.2: A big picture of NLOS identification

\subsection{NLOS identification: a statistical detection problem}

In essence, NLOS identification is a statistical detection problem. NLOS and LOS conditions are considered as two hypothesis. The NLOS identification process can be depicted using Figure 1.2. The source block in Figure 1.2 generates one of the possible outputs, i.e., NLOS or LOS hypothesis. Then, random observations are generated based on the conditional probability density function (pdf) $f\left(\cdot \mid H_{0}\right)$ or $f\left(\cdot \mid H_{1}\right)$. In the decision rule block, the likelihood ratio $\Lambda(r)$ is compared to a threshold and then a decision is made on whether LOS or NLOS hypothesis is true. The main task in NLOS identification is to find out metrics that differentiate NLOS and LOS and enable a binary hypothesis test to identify NLOS conditions.

\subsection{Different categories of NLOS identification techniques}

NLOS identification techniques can be cooperative or non-cooperative. Cooperative techniques use multiple nodes that are geographically distributed in an environment to identify 
a NLOS measurement. Non-cooperative NLOS identification are based on single node channel measurements. The non-cooperative methods can be divided into three groups:

1. based on the range (TOA) statistics: The range is the product of TOA and the speed of the light. If the LOS path is available, the estimated range is affected by the TOA estimation error, and therefore is Gaussian distributed. But for NLOS situation, the estimated range is positively biased and has non-Gaussian distribution. In addition, NLOS range measurements tends to have a larger variance compared to LOS range measurements;

2. based on channel characteristics: This includes received signal power, Rician Kfactor and features extracted from the power delay profile; and

3. hybrid methods: These methods explore the consistency between the TOA measurement and path loss for LOS/NLOS, and the consistency between the direction of departure (DOD) and DOA.

\subsection{The main idea}

Prior techniques mainly use the statistics attainable by one antenna element to enable NLOS identification. Examples of these statistics are range statistics [14], Rician $K$-factor [15], multipath auto correlation [16] and etc. The performance of these techniques are limited because the features extraction using one antenna element does not allow high performance 


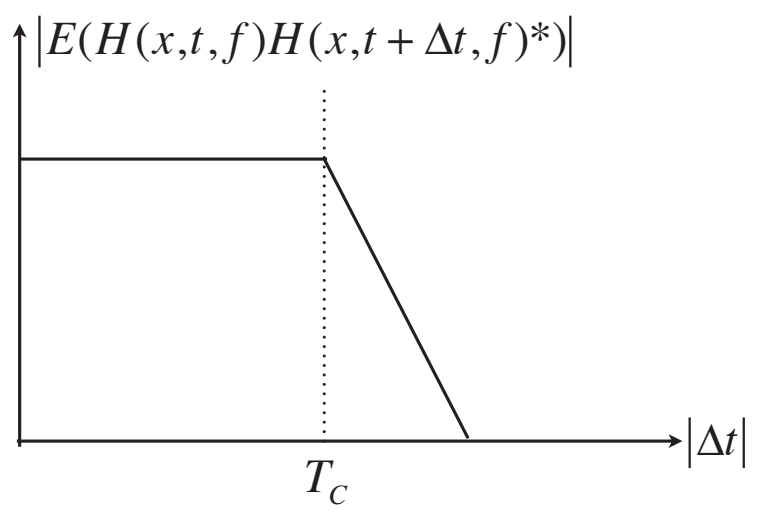

Figure 1.3: Channel correlation across time

NLOS identification. However, in DOA-based wireless localization systems, multiple antenna elements are available. The spatial channel information exclusively available in multiple antenna systems has the potential to identify NLOS.

A general impulse response of the radio channel of multiple antenna systems is characterized as time-varying, frequency-selective and space-selective [17]. Assuming the location of the receiver is denoted by a vector $x \in \mathbb{R}^{3}$, the observation time instant is denoted by $t$ and the multipath delay is denoted by $\tau$, the channel impulse response in NLOS conditions can be represented by [17]

$$
h(x, t, \tau)=\sum_{l=1}^{L} a_{l} \exp \left\{j 2 \pi \lambda^{-1}\left(\Omega_{l} \cdot x\right)\right\} \exp \left(j 2 \pi v_{l} t\right) \delta\left(\tau-\tau_{l}\right)
$$

where $\lambda$ is the wavelength, $a_{l}$ is the complex amplitude, $\Omega_{l}$ is the incidence direction, $v_{l}$ is the Doppler frequency and $\tau_{l}$ is the delay of the $l^{\text {th }}$ impinging wave. Applying Fourier transform to $h(x, t, \tau)$ with respect to the delay $\tau$, the channel frequency response is obtained and denoted by $H(x, t, f)$. 


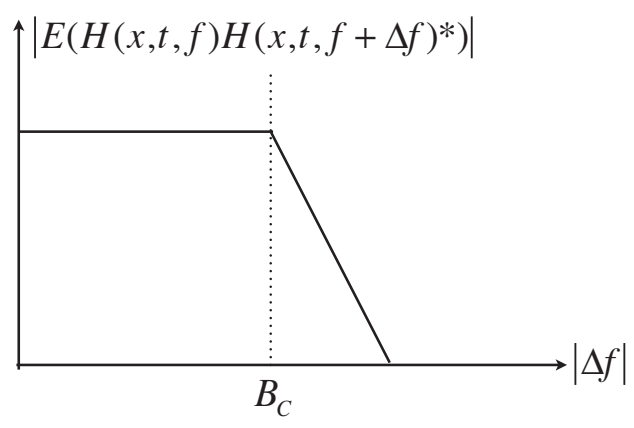

(a)

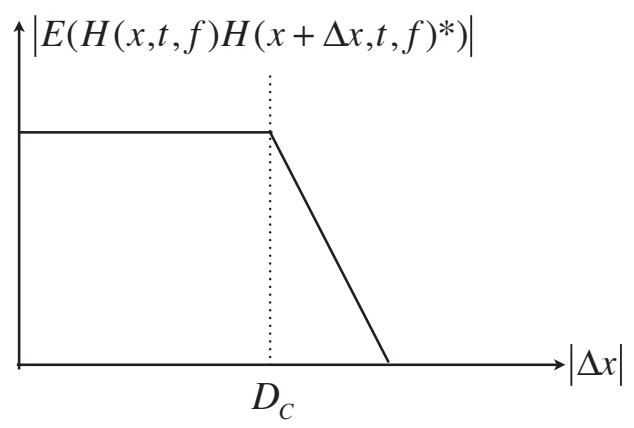

(b)

Figure 1.4: (a) Channel correlation across frequency (b) Channel correlation across distance

The channel frequency response $H(x, t, f)$ varies with the location, time and frequency. The correlation of channel frequency response at a time separation $\mid E(H(x, t, f) H(x, t+$ $\left.\Delta t, f)^{*}\right) \mid$ is depicted in Figure 1.3. In Figure 1.3, $T_{c}$ denotes the coherence time. It is seen that when $|\Delta t|>T_{c}$, the correlation drops to a low level. Let $B_{c}$ and $D_{c}$ denotes the coherence bandwidth and coherence distance, respectively. The correlation at a frequency separation $\left|E\left(H(x, t, f) H(x, t, f+\Delta f)^{*}\right)\right|$ and the correlation at a space separation $\left|E\left(H(x, t, f) H(x+\Delta x, t, f)^{*}\right)\right|$ are shown in Figure 1.4.

A similar phenomenon can be observed that when $|\Delta f|>B_{c}$ and $|\Delta x|>D_{c}$, the correlation would drop to a low level. In LOS conditions, the correlation of channel frequency response would be the sum of the LOS component correlation and the NLOS correlation. Note that the envelope of LOS component correlation would not change with $\Delta t, \Delta f$ and $\Delta x$. Therefore, when $\Delta t, \Delta f$ or $\Delta x$ is big enough, the channel correlation under NLOS conditions tends to zero while the channel correlation under LOS conditions stays around the value of LOS component correlation. These facts form the basis of NLOS identification 
techniques proposed in this dissertation.

\subsection{MIMO-OFDM basics}

As mentioned earlier, in this dissertation, we propose NLOS identification measures that are based on statistical features that can be extracted using MIMO-OFDM systems. The use of MIMO technology in combination with OFDM, i.e., MIMO-OFDM has been adopted as the solution for emerging wideband wireless standards. These include IEEE 802.11n - the next generation standard for wireless local networking, IEEE 802.16e - a new standard for metropolitan area networks, and 3GPP Long Term evolution (LTE) - the next generation standard for cellular networking. In the following, basics of OFDM and MIMO technology are briefly described.

\subsubsection{OFDM basics}

In wideband systems, the symbols received in time domain are subject to inter-symbol interference (ISI): delayed replicas of previous symbols interfere with the current symbol. ISI occurs in multi-path channels. Wireless channels represent a filtering effect that is characterized by the channel coherence bandwidth. ISI is typically generated when the symbol bandwidth is in the order or higher than the channel coherence bandwidth.

Many techniques are proposed to tackle ISI effects. Equalization is an ISI removal tech- 


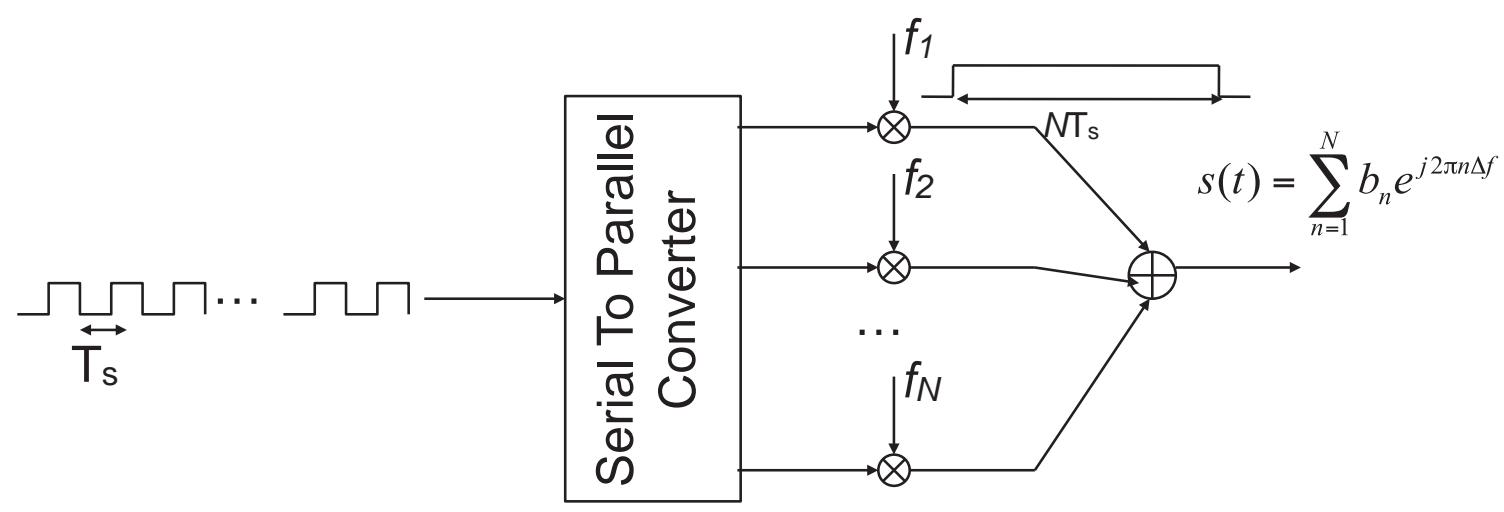

Figure 1.5: Multicarrier transmitter

nique that is implemented in the receiver only. Equalization techniques are realized via complex signal processing techniques that increase the complexity and power consumption of the receiver. In late 1990's multicarrier modulation was introduced as a novel approach that avoids ISI to occur in the first place. In multicarrier modulation, $N$ symbols created by the channel coder are serial to parallel converted, and each symbol is transmitted over a unique carrier. The half power bandwidth of original symbols is $1 / T s$, while the bandwidth of them after serial to parallel conversion would be $1 /(N T s)$. Figure 1.5 represents the operation of multicarrier systems.

By selecting the number of carriers $(N)$ properly, the original wideband symbols are converted to narrowband counterparts, such that the bandwidth of these narrowband signals becomes smaller than the channel bandwidth. In this case, ISI is avoidable. The frequency separation between these carriers is properly selected to maintain orthogonality across the carriers and avoid ISI creation at the receiver as well.

OFDM is the discrete implementation of multicarrier modulation. OFDM systems are 


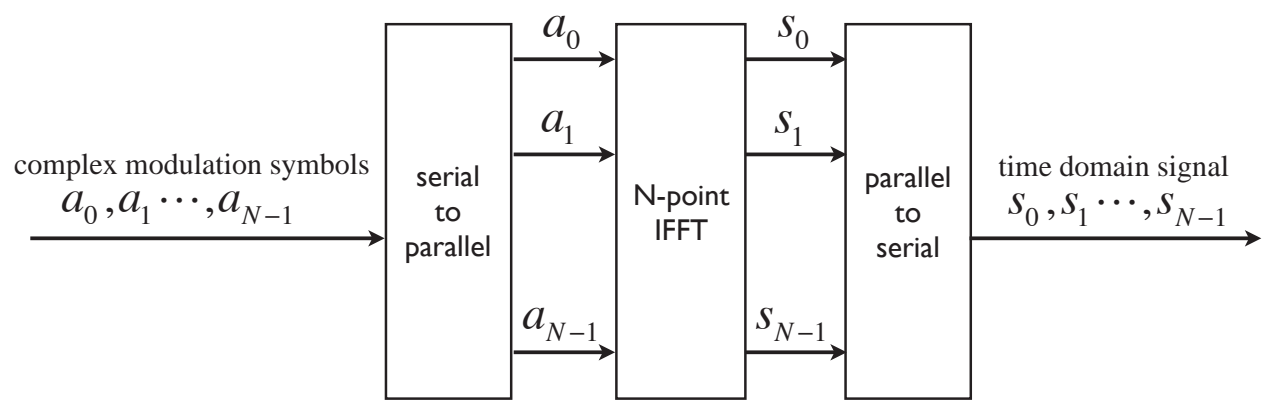

(a) OFDM transmitter

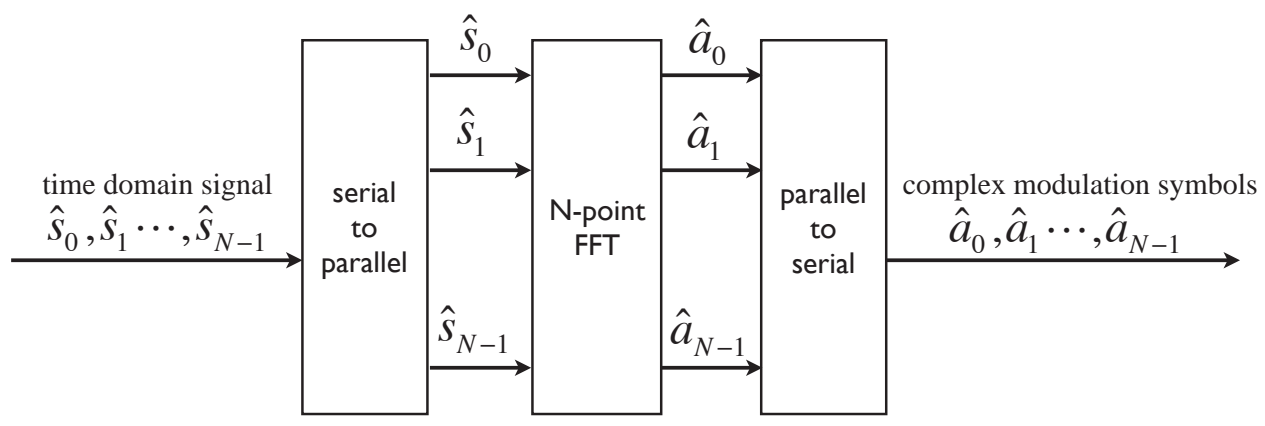

(b) OFDM receiver

Figure 1.6: OFDM with IFFT/FFT implementation

simply implemented by an IFFT operation at the transmitter and an FFT operation at the receiver. Figure 1.6 represents the implementation of an OFDM system. It is depicted that OFDM systems are mainly implemented via simple digital operations. Therefore, OFDM modulation reduces receiver complexity.

\subsubsection{MIMO basics}

MIMO systems use multiple antenna elements at the transmitter and receiver to maintain high throughput and reliable communication.

The capability of high throughput communication in MIMO systems is realized by spa- 


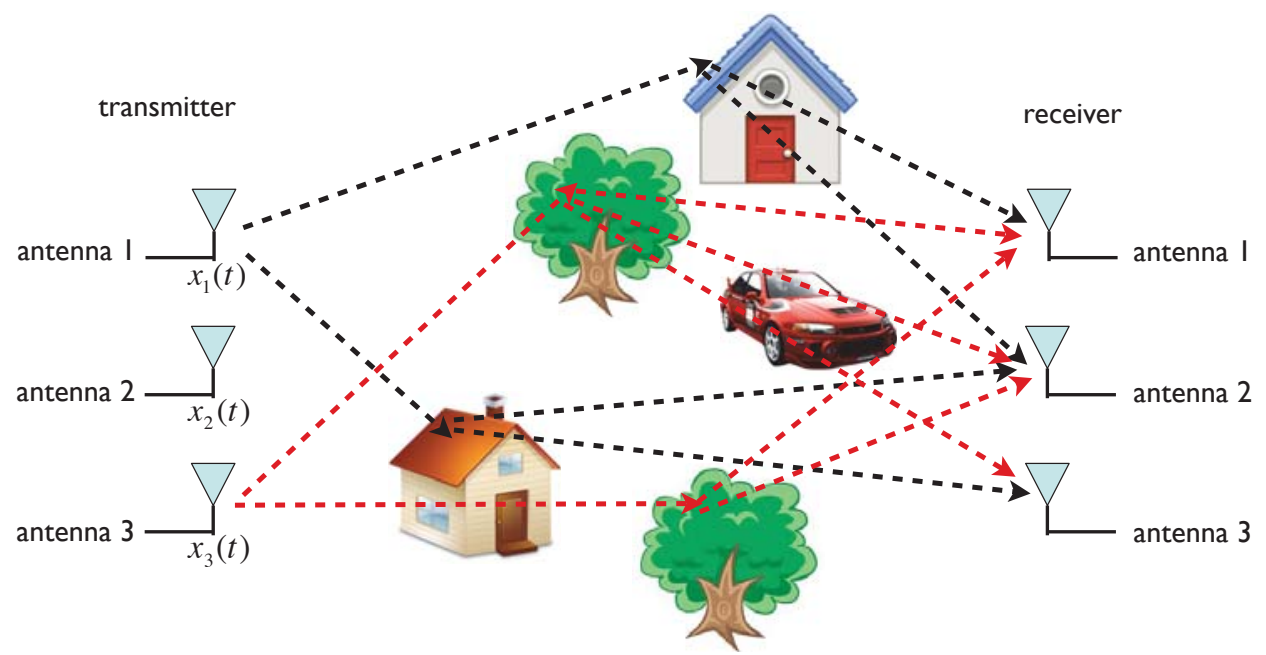

Figure 1.7: MIMO systems

tially multiplexing several independent data steams onto the MIMO channel under suitable channel fading conditions. For example, in Figure 1.7, three independent data streams denoted by $x_{1}(t), x_{2}(t)$ and $x_{3}(t)$ are simultaneously transmitted via transmit antenna 1,2 and 3 , respectively. The receiver equipped with multiple antennas is able to correctly detect the three transmitted data streams. The throughput or capacity of a MIMO channel with $n$ transmit and receive antennas is proportional to $n$. Note that this throughput is $n$ times higher than that of single antenna systems.

One of the important problems in wireless communication systems is multi-path fading. In a multi-path channel the signal received through multiple paths might be added together destructively. This reduces the performance of receivers in wireless systems. Diversity combining techniques are proposed to tackle fading in wireless channels. Diversity refers to the transmission of signals over multiple independent channels. Proper combination of the signals from these transmission channels yields a resultant with greatly reduced 
severity of fading and accordingly improves reliability of transmission. Different types of diversity schemes include frequency diversity, time diversity, and space diversity. The reliable communication in MIMO systems is enabled by exploiting the space diversity. In Figure 1.7, the data stream $x_{1}(t)$ is received by each receive antenna. The received signals of $x_{1}(t)$ at each receive antenna are mutually independent when the channel coefficients from the transmit antenna 1 to each receive antenna are mutually independent. The channel independency across receive antennas is held for the propagation channels containing a great amount of spatially distributed scatterers which scatter radio waves.

\subsection{Chapter contributions}

Chapter 2 reviews NLOS identification techniques in the literature. Chapter 3, Chapter 4, Chapter 5 and Chapter 6, collectively, propose four new NLOS identification techniques for multiple antenna systems.

Chapter 2 reviews many cooperative and non-cooperative NLOS identification techniques. Cooperative techniques use multiple nodes to identify NLOS measurements. Non-cooperative NLOS identification is based on single node channel measurements, such as range statistics and features extracted from the power delay profile. In the non-cooperative methods, various techniques applied to narrow/wide band systems and ultra-wide-band (UWB) systems are discussed. Moreover, the advantages and disadvantages, the complexity and performance of each technique are discussed. 
In Chapter 3, channel spatial correlation is proposed to identify NLOS scenarios for narrow band multiple antenna systems. In order to obtain the channel spatial correlation, a new multi-user MIMO channel model for rough surface scattering is proposed. Here, the scatterers are modeled as random rough surfaces: Any point on rough surfaces scatters the incident wave into any given direction with certain probability. This leads to correlation across antenna elements within one user and across users. The closed form expressions for the intra-user (point-to-point) and inter-user (multi-user) correlation of channel coefficients across antenna elements are derived. The correlation is affected by the distance between transceivers, the geometry of the rough surfaces and the roughness of the surfaces. The impact of these parameters is evaluated.

In Chapter 4, the phase difference statistics across two antenna elements is proposed to identify NLOS scenarios for narrow band multiple antenna systems. This technique assumes that the phases at two antenna elements are uncorrelated and this is held when the antenna separation is greater than the coherence distance. The validity of this assumption is verified via channel modeling simulations. A phase wrapping selection algorithm is proposed to calculate the phase difference variance across two antenna elements. A theoretical relationship is maintained between the phase difference variance and the Rician $K$-factor. Then, a hypothesis test on the $K$-factor is formed to identify NLOS situations. The prior distributions of $K$-factor under LOS and NLOS conditions, and the $K$-factor threshold which are used to distinguish LOS and NLOS situation are derived. The impact of shadowing on the performance of the proposed NLOS identification method is studied. 
The performance of the proposed phase difference based $K$ estimator is compared with that of the envelope-based $K$ estimator.

Chapter 5 and Chapter 6 propose a non-line-of-sight (NLOS) identification techniques that exploit space-frequency channel correlation and space-time-frequency channel correlation of wide band systems using multi-input multi-output (MIMO) orthogonal frequency division multiplexing (OFDM) signaling. Here, space-time correlation refers to the correlation across antenna elements and time, and frequency correlation refers to the correlation across subcarriers. Two groups of metrics are proposed for NLOS identification. The first group of metrics are based on space-frequency channel correlation and these metrics require minimal variation of spatial correlation across different multi-path components. The channel model satisfying this requirement is studied. For the channel models fail to meet minimal variation requirement, a second group of metrics based on space-time-frequency channel correlation are applied. The probability of detection performance of the new NLOS identification method is investigated. 


\section{Chapter 2}

\section{A Review on NLOS Identification Techniques in the Literature}

When line-of-sight (LOS) is not available, i.e., in non-LOS (NLOS) conditions, directionof-arrival (DOA) and time-of-arrival (TOA) techniques would involve with considerable errors. To address this problem, many techniques have been proposed to identify LOS conditions. If the NLOS situations are identified, the corresponding measurements should be excluded from the localization process to eliminate the corresponding NLOS error.

This chapter reviews many NLOS identification techniques. NLOS identification techniques can be categorized into cooperative and non-cooperative. Cooperative techniques use multiple nodes to identify NLOS measurements. Non-cooperative NLOS identification is based on single node channel measurements. These techniques are based on: (1) the 
range (TOA) statistics; (2) channel characteristics, such as received signal power, Rician Kfactor and features extracted from the power delay profile; and (3) the consistency between the TOA measurement and path loss for LOS/NLOS, and the consistency between the direction of departure (DOD) and DOA. In the second group, suitable channel characteristics used in narrow/wide band systems and ultra-wide-band (UWB) systems are discussed.

\subsection{Introduction}

This chapter reviews many NLOS identification techniques in the literature. In addition, the advantages and disadvantages, the complexity and performance of each technique are discussed.

The rest of this chapter is organized as follows. In Section 2.2, cooperative NLOS identification is introduced. In Section 2.3, NLOS identification techniques based on the range statistics are presented. In Section 2.4, NLOS identification techniques based on channel characteristics are presented. Section 2.5 presents miscellaneous NLOS identification methods. In Section 2.6, the NLOS identification methods presented in previous sections are compared in terms of requirements and performance. Section 2.7 concludes this chapter. 


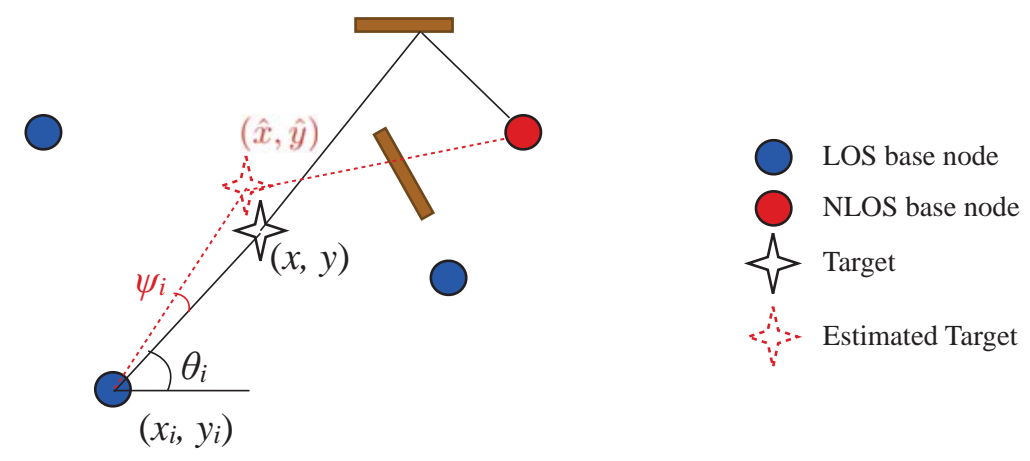

Figure 2.1: NLOS identification based on DOA residual testing

\subsection{Cooperative NLOS identification}

When multiple based nodes are involved in determining the position of a target, base nodes (BNs) located in the LOS of a target produce consistent localization results, but BNs in the NLOS condition produce inconsistent localization results. Because inconsistent NLOS measurements tend to have large residuals, residual testing is an approach that can identify NLOS measurements. Many residual testing techniques have been proposed in the literature. Here we present a summary of those techniques.

\subsubsection{DOA residual testing}

This approach has been proposed in [9]. In this method, it is assumed that there are $N$ BNs at known locations $\left(x_{i}, y_{i}\right), i=1 \ldots N$ as illustrated in Figure 2.1. All BNs can measure DOAs of the signal transmitted by the target. Let $\theta_{i}$ be the DOA measured by $\mathrm{BN} i$. The NLOS identification procedure consists of the following steps: 
1. Find the maximum likelihood position $(\hat{x}, \hat{y})$ of the target using all DOA estimates.

2. Calculate the DOA residual $\psi_{i}$ (see Figure 2.1) which is the absolute difference of $\theta_{i}$ and the DOA obtained via the estimated target position $(\hat{x}, \hat{y})$.

3. Select the NLOS BNs whose $\psi_{i}>1.5 R M S(\psi) . R M S(\psi)$ denotes the root mean square of $\psi_{i}$.

After excluding the NLOS BNs, the maximum likelihood position of the target is estimated again and the positioning accuracy is improved. The simulation result shows that when there are 4 LOS BS and 1 NLOS BS, applying NLOS identification can cut the root mean square positioning error greatly, from $1.2 \mathrm{~km}$ to $125 \mathrm{~m}$, which meets E911 requirements.

\subsubsection{Time-difference-of-arrival (TDOA) residual}

This approach defined in [18] takes into account the fact that NLOS error is always positive. Assuming the measurement noise under the LOS condition is Gaussian distributed, the residual $e_{i}$ is expressed as

$$
e_{i}=0.5+0.5 \operatorname{erf}\left(\frac{m_{i}-f_{i}(\hat{\theta})}{\sqrt{2} \sigma_{i}}\right)
$$

In (2.1), $\operatorname{erf}(\cdot)$ is the error function defined as $\operatorname{erf}(x)=(2 / \sqrt{\pi}) \int_{0}^{x} e^{-t^{2}} d t, m_{i}$ represents the TDOA measurement of $\mathrm{BN} i$ and the reference $\mathrm{BN}, \hat{\theta}$ is the estimated target position using all measurements, $f_{i}(\hat{\theta})$ is the BN $i$ 's TDOA given the target position $\hat{\theta}$ and $\sigma_{i}$ is the 
square root of the measurement noise variance. The higher this residual, the more likely the measurement $m_{i}$ is biased by NLOS error(s). Then the residual is compared with the threshold $\lambda$ and those BNs with residuals larger than $\lambda$ are labeled as NLOS BNs. When there is only one NLOS BN out of a total of $6 \mathrm{BNs}$, the identification probability can attain 0.79. However, this probability decreases when less BNs are available or more NLOS BNs are present.

\subsubsection{Residual distribution testing}

This approach finds the set of LOS BNs [19]. let there be $N$ BNs which use TOA estimates to locate target nodes. The minimum number of BNs required for obtaining an estimate of the target position is three. Therefore, there are a number of position estimates corresponding to different combinations of BNs and the total number is $S=\sum_{i=3}^{N}{ }_{N} C_{i}$ where ${ }_{N} C_{i}$ represents the number of $i$-element combination out of $N$ elements, i.e., ${ }_{N} C_{i}=\frac{N !}{i !(N-i) !}$. Then the normalized residuals are defined as

$$
\begin{aligned}
\chi_{x}^{2}(k) & =\frac{[\hat{x}(k)-\hat{x}]^{2}}{B_{x}(k)} \\
\chi_{y}^{2}(k) & =\frac{[\hat{y}(k)-\hat{y}]^{2}}{B_{y}(k)}, \quad k=1, \ldots S-1
\end{aligned}
$$

where $(\hat{x}(k), \hat{y}(k))$ represents the target position estimate using the $k^{\text {th }} \mathrm{BN}$ combination, $(\hat{x}, \hat{y})$ is the estimation involving all $\mathrm{BNs}$, and $B_{x}(k), B_{y}(k)$ are the approximations of the Cramer-Rao lower bound (CRLB) on the estimation error of the respective $x$ and $y$ target 
coordinates. If all $N$ BNs are in the LOS condition, $\frac{\hat{x}(k)-\hat{x}}{\sqrt{B_{x}(k)}}$ and $\frac{\hat{y}(k)-\hat{y}}{\sqrt{B_{y}(k)}}$ are approximately Gaussian distributed with zero mean and unit variance. Therefore, the normalized residuals in (2.2) have an approximate central $\chi^{2}$ pdf. If one or more BNs are in NLOS condition, the means of $(\hat{x}(k)-\hat{x})$ and $(\hat{y}(k)-\hat{y})$ are biased by the NLOS measurement. Therefore, the normalized residuals have a non-central $\chi^{2}$ pdf. The non-central $\chi^{2}$ distribution can be detected using the fact that the probability of the central $\chi^{2}$ distributed random variable being greater than 2.71 is 0.02 . Thus, the appearance of a value higher than the threshold $T H=2.71$ indicates that there is one or more NLOS measurements with high likelihood. The identification steps are as follow:

1. The normalized residuals defined in (2.2) are calculated for a total number of $N$ BNs.

2. The residuals are compared to $T H=2.71$. Then, the cases $\chi_{x}^{2}>T H$ and $\chi_{y}^{2}>T H$ are counted.

3. If less than $10 \%$ of the residuals are above $T H$, then the number of LOS BN is $D=N$. Otherwise, the test moves to $(N-1)$ BNs.

4. This process stops when it has determined a $D$, or when $D=3$.

5. The excluded BNs are identified as NLOS.

Simulations show that the location mean square error (MSE) is close to the CRLB for more than 4 LOS BNs. But for 3 LOS BNs, identifying the NLOS BNs is difficult and the location MSE is higher. 
The pros and cons of the cooperative NLOS identification approaches are summarized as

Pros

$\dagger$ NLOS conditions are selected in a way that the position estimation error can be reduced.

\section{Cons}

$\uparrow$ In order to correctly detect NLOS measurements in residual testing, there should be at least 4 LOS BNs, while in real environments rarely enough LOS BNs are available.

$\dagger$ The locations of all BNs are required.

$\dagger$ The computation complexity is high and it increases with the number of BNs.

In Section 2.3, single node identification methods are studied. Single node methods do not require LOS BNs or the locations of any BN and their complexity is not high in general.

\subsection{NLOS identification based on the range statistics}

Range refers to the distance between the BN and the target. Range is computed by multiplying TOA and the speed of light. NLOS can be identified based on the features derived 
from the time series of the range estimates $[14,20,21,22]$ or the features from the range estimates across different frequency bands [23].

In this section, first, the methods that are based on range measurements over time are investigated and next the methods that are based on range measurement over different frequency bands are studied.

\subsubsection{Techniques based on range measurements over time}

For LOS and NLOS situations, the $i^{t h}$ range measurements can be modeled as

$$
\begin{aligned}
& \text { LOS: } \quad r_{i}=d_{i}+n_{i} \\
& \text { NLOS: } r_{i}=d_{i}+n_{i}+e_{i}, \quad i=1, \ldots, N
\end{aligned}
$$

where $d_{i}$ is the true LOS range, $n_{i}$ is the measurement noise, and $e_{i}$ is the NLOS error. In (2.3), $n_{i}$ is modeled as Gaussian distributed with zero mean and variance $\sigma^{2}$, and $e_{i}$ is modeled by a random variable which is exponentially [24] or Gaussian distributed [20] with positive mean $\mu_{e}$ and variance $\sigma_{e}^{2}$. Normally, $n_{i}$ and $e_{i}$ are independent. Empirical measurement in [25] shows that the NLOS error is more irregular than the measurement noise, which results in $\sigma_{e}^{2}>\sigma^{2}$. Therefore, a hypothesis test can be formed based on the pdf or the variance of the estimated range. Note that this technique usually assumes that the target is moving and thus the positions of reflectors between transceivers change with time. 
Therefore, the NLOS range measurements change over time and have a larger variance.

The testing based on range variance is used when there is not a priori information about the NLOS error (such as pdf, mean and variance) [20]. The measurement noise variance $\sigma^{2}$ is usually known, as it is determined by the range estimation method. Let $\hat{\sigma}^{2}$ denote the estimated range variance and the hypothesis testing is given by

$$
\begin{array}{ll}
H_{0}: & \hat{\sigma}^{2}=\sigma^{2}(\text { LOS condition }) \\
H_{1}: & \hat{\sigma}^{2}>\sigma^{2}(\text { NLOS conditon })
\end{array}
$$

The decision rule is

$$
\begin{gathered}
H_{1} \\
\hat{\sigma}^{2} \underset{\eta}{\gtrless} \\
H_{0}
\end{gathered}
$$

The threshold $\eta$ may vary with the availability of a priori information: If only the noise variance is known, $\eta$ is $\sigma^{2}$; If the NLOS error variance $\sigma_{e}^{2}$ is known, $\eta=\sigma_{e}^{2} / 2$; The threshold $\eta$ can also depend on the typically known maximal velocity of the object [26]. In reality, the true range $d_{i}$ varies with time $i$ and a polynomial fitting is used to compute the range variance $\hat{\sigma}^{2}$ [14]. The true range is reconstructed via the polynomial fitting, and the 
reconstructed range is denoted by $s_{i}$. Then, the range variance can be calculated

$$
\hat{\sigma}^{2}=\sqrt{\frac{1}{N} \sum_{i=1}^{N}\left(s_{i}-r_{i}\right)^{2}}
$$

The testing based on the range distribution can be divided into two groups: parametric and non-parametric. In parametric methods, part or complete a priori information is known such as the likelihood of LOS and NLOS error pdf. Assuming Gaussian NLOS error, reference [20] discusses parametric NLOS identification and forms a couple of likelihood ratio tests for different levels of a priori information. For example, if the likelihood of LOS and the pdf of NLOS error are known, and the true LOS range is not known, a generalized likelihood ratio test (GLRT) can be used, which corresponds to

$$
\Lambda_{g}(\mathbf{r})=\frac{\max _{d+\mu_{e} f_{n l o s}(\mathbf{r})} \stackrel{H_{1}}{\max _{d} f_{l o s}(\mathbf{r})} \underset{P(L O S)}{\gtrless(N L O S)}}{H_{0}}
$$

where $\mathbf{r}$ is a vector of range measurements, $\mathbf{r}=\left[r_{1}, \ldots, r_{N}\right], d$ is the true LOS range, $f_{\text {nlos }}$ and $f_{\text {los }}$ represent the pdfs of LOS and NLOS range measurement, $P(L O S)$ and $P(N L O S)$ are the likelihoods of LOS and NLOS hypotheses, respectively.

Because the NLOS distribution is site-specific, its characterization is very difficult. In these cases, the non-parametric technique which does not assume the knowledge of NLOS error 
statistics can be used in NLOS identification. In [22, 21], it is assumed that it is only known that the NLOS error is not Gaussian. Tests for the normality of the range measurements are developed in [21]. In [22], a metric measuring the distance between two pdfs are introduced and the distances between the candidate LOS range pdfs and the range measurements pdf are computed. Then, LOS is decided when the minimum pdf distance is smaller than the threshold, and NLOS is decided otherwise.

\subsubsection{Techniques based on the range measurements over different frequency bands}

Based on channel measurements in a typical indoor environment, the authors of [23] show that under LOS condition estimated ranges are similar across sub bands, but under NLOS condition they are drastically different across sub bands. The difference of ranges across sub bands is due to the different propagation characteristics across sub bands: higher operational frequency means lower penetration capabilities. In other words, signal at higher frequency bands may not penetrate blockage and experience a NLOS propagation while signal at lower frequency bands may penetrate blockage and still experience a LOS propagation. Thus, the LOS ranging measurements over sub bands have a small variance and the NLOS ranging measurements over sub bands have a large variance. Let $\sigma$ denote the standard deviation of the ranging measurements and let $f_{\text {nlos }}$ and $f_{l o s}$ be the pdfs of $\sigma$ for 
respective hypothesis. The decision rule is

$$
\begin{array}{cc}
\frac{f_{\text {nlos }}(\sigma)}{f_{\text {los }}(\sigma)} & \text { NLOS } \\
& \gtrless \sigma_{\text {sh }} \\
& \text { LOS }
\end{array}
$$

where $f_{\text {los }}, f_{\text {nlos }}$ and the threshold $\sigma_{\text {sh }}$ need to be determined experimentally. This method can be implemented on multi-band orthogonal frequency division multiplexing (OFDM) systems. The multi-band approach requires a frequency hopping capable RF front end and therefore the cost and the complexity are higher. It would be a cheaper solution to combine radio ranging signal and low frequency sound (such as in [27]). How their ranging is different under different channel conditions needs to be investigated.

A summary of this part is given in Table 2.1. A general disadvantage of range statistics based methods is the latency (about 5 seconds) due to using time series of the range estimates. Therefore in Section 2.4, faster NLOS detection methods are studied. When the $\mathrm{BN}$ and the target are both stationary, which means that the signal traveling path does not change, this method would fail because the range statistics will not differ considerably for LOS and NLOS situations. 
Table 2.1: A summary on range statistics based methods

\begin{tabular}{|l|l|l|l|}
\hline & $\begin{array}{l}\text { Estimated range } \\
\text { pdf }\end{array}$ & Range variance & $\begin{array}{l}\text { Range variance across } \\
\text { sub bands }\end{array}$ \\
\hline LOS & Gaussian & Small & Small \\
\hline NLOS & Non-Gaussian & Large & Large \\
\hline \multirow{2}{*}{ Cons } & $\begin{array}{l}\text { Not for stationary } \\
\text { BN and target }\end{array}$ & $\begin{array}{l}\text { Not for stationary } \\
\text { BN and target }\end{array}$ & $\begin{array}{l}\text { Only for multi-band } \\
\text { UWB }\end{array}$ \\
\cline { 2 - 4 } & \multicolumn{3}{|c|}{ Latency } \\
\hline
\end{tabular}

\subsection{NLOS identification based on channel characteristics}

This section investigates NLOS identification approaches based on channel characteristics.

Almost all channel characteristics mentioned here are extracted from the power delay profile of the received signal.

Since the power delay profile exhibits differently for systems with different bandwidth, this discussion includes methods for narrow and wideband systems and UWB systems.

\subsubsection{Narrow and wideband systems}

In this case, the power envelope distribution of the received signal can be used to identify NLOS [28], because the power distribution of the first arriving path is usually modeled as Rayleigh fading for NLOS condition and Rician fading for LOS condition [29]. Here is the identification process:

1. Estimate the pdf of the first arriving path power. To correctly estimate this pdf, a 
set of independent fading coefficients are needed. The fading coefficients would be considered independent if they are separated by at least a coherence time.

2. Compare the estimated pdf to some reference pdfs, such as Rayleigh or Rician, via Pearson's test statistic [15] or Kolmogorov-Smirnov test [28].

3. Form a hypothesis test on the comparison result and make a decision.

This method has two disadvantages: (1) the observation interval should be long enough to compute the accurate pdf of the first path power. As reported in [15], this time interval is in the order of one second; and (2) when the LOS component is much smaller than the NLOS component in the first path, it is difficult to distinguish the power distribution under LOS condition from the distribution under NLOS condition, i.e., Rayleigh.

In order to further reduce the observation time, an approach based on the Rician $\mathrm{K}$ factor of the first arriving path is proposed in $[30,15]$. Rician $K$ factor is defined as the ratio of LOS and NLOS component powers. When there is no LOS component (NLOS condition), $K=0$ by its definition. When LOS component exists, $K>0$. In [15], the Rician $K$ factor is estimated, denoted by $\hat{K}$ and the LOS state is weighted according to a pre-defined scale:

$$
\begin{array}{ll}
\text { if } \hat{K}>K_{\max } & \text { decide LOS } \\
\text { if } K_{\min }<\hat{K}<K_{\max } & \text { the probability of LOS is: }\left(\hat{K}-K_{\min }\right) /\left(K_{\max }-K_{\min }\right) \\
\text { if } \hat{K}<K_{\min } & \text { decide NLOS }
\end{array}
$$


In [30], a simplified hypothesis testing is used:

$$
\begin{aligned}
& \hat{K}>1, \quad \text { decide LOS } \\
& \hat{K}<1, \quad \text { decide NLOS }
\end{aligned}
$$

The time required to estimate $K$ is around 10 milliseconds reported in [15].

Another approach to identify NLOS depends on the autocorrelation properties of each multipath component $[31,16]$. The autocorrelation of multipath components indicates how the corresponding fading coefficient varies with time. If fading coefficients vary fast, the autocorrelation is low. Otherwise, the autocorrelation is high. The NLOS multipath component coefficient usually varies fast, since it consists of numerous time-varying irresolvable paths. The presence of LOS component in the first path provides higher autocorrelation as compared to the other paths which do not include LOS component, because the LOS component coefficient has a deterministic structure and varies slowly. If there is no LOS component in the first path, the autocorrelation of the first path would be comparable to the following paths. This observation can be used to identify the existence of the LOS component.

\subsubsection{Ultra-wide-band (UWB) systems}

UWB enables precise ranging and localization via incorporating extremely short duration pulses. In this case, the multipath components of the received signal can be well resolved. Therefore, it is a very promising technique for indoor localization. Moreover, the UWB 
channel models have been intensively characterized for LOS and NLOS channel conditions [32], based on which some metrics distinguishing LOS and NLOS are studied.

In [26], a confidence metric is given as a function of the amplitude $\alpha_{1}$ and the arrival time $\tau_{1}$ of the first path, and the strongest path amplitude $\alpha_{\max }$ and the respective arrival time $\tau_{\max }$. Based on the observation that compared to the first path, the subsequent multipath components should have lower power in the LOS case and vice versa in the NLOS case, the confidence metric would be high for LOS case and low for NLOS case. Another similar approach is proposed in [33]. Here, the first path power $\left|\alpha_{1}\right|^{2}$ and the delay of the strongest path, i.e., $\tau_{\max }-\tau_{1}$ are used to form a joint likelihood ratio test as follows:

$$
\begin{aligned}
& \text { LOS } \\
& J\left(\left|\alpha_{1}\right|^{2}, \tau_{\max }-\tau_{1}\right)=\frac{f_{\text {los }}\left(\left|\alpha_{1}\right|^{2}\right)}{f_{\text {nlos }}\left(\left|\alpha_{1}\right|^{2}\right)} \times \frac{f_{\text {los }}\left(\tau_{\max }-\tau_{1}\right)}{f_{\text {nlos }}\left(\tau_{\max }-\tau_{1}\right)} \gtrless 1 \\
& \text { NLOS }
\end{aligned}
$$

A disadvantage of these two methods is that they may mistakenly detect non-dominant direct path (NDDP) channel condition as NLOS, because in NDDP cases the direct path (LOS) is not the strongest but still detectable by an appropriate receiver architecture.

A method based on the change of signal power is proposed in [26]. The principle is that a sudden decrease of the maximum signal power $\left|\alpha_{\max }\right|^{2}$ could indicate the movement from a LOS into a NLOS condition, and vice versa. The LOS and NLOS states are detected when the transition between LOS and NLOS occurs, therefore it is not suitable for the case when 
the channel stays at one state for a long time.

NLOS identification methods based on RSS test are proposed in [34, 35]. RSS is defined as the total received power of the received signal. The received signal $h(t)$ is

$$
h(t)=\sum_{l=1}^{L} \alpha_{l} \delta\left(t-\tau_{l}\right)
$$

where $L$ is the total number of multi-paths, $\alpha_{l}$ is the amplitude of the $l^{\text {th }}$ multi-path, and $\tau_{l}$ is the delay of the $l^{\text {th }}$ multi-path. Then, RSS is represented by

$$
R S S=\sum_{l=1}^{L}\left|\alpha_{l}\right|^{2}
$$

RSS can be easily measured by most wireless devices. The estimated RSS has been modeled as a lognormal random variable with different variances in LOS and NLOS scenarios [34]. Then a likelihood ratio test similar to (2.8) can be applied to determine LOS or NLOS. In [35], RSS is modeled by Weibull distribution based on measurements.

Other metrics can be extracted from the received multi-path signal $h(t)$, and similar hypothesis testings can be formed to identify NLOS. Those metrics include mean excess delay, Delay spread, kurtosis, skewness [34, 12, 36, 37], and they are defined as follows: 
mean excess delay

$$
\tau_{m}=\frac{\int_{-\infty}^{\infty} t|h(t)|^{2} d t}{\int_{-\infty}^{\infty}|h(t)|^{2} d t}
$$

where $h(t)$ is defined in (2.12)

\section{delay spread}

$$
\tau_{r m s}=\frac{\int_{-\infty}^{\infty}\left(t-\tau_{m}\right)^{2}|h(t)|^{2} d t}{\int_{-\infty}^{\infty}|h(t)|^{2} d t}
$$

Here, $\tau_{m}$ is defined in $(2.14)$

\section{kurtosis}

$$
\kappa=\frac{E\left[\left(|h(t)|-\mu_{|h|}\right)^{4}\right]}{\sigma_{|h|}^{4}}
$$

where $E(\cdot)$ denotes expectation over delay, and $\mu_{|h|}$ and $\sigma_{|h|}$ are the mean and standard deviation of $|h(t)|$, respectively.

\section{skewness}

$$
s=\frac{E\left[\left(|h(t)|-\mu_{|h|}\right)^{3}\right]}{\sigma_{|h|}^{3}}
$$

Some of the above metrics can be combined as shown in (2.11) to achieve higher identification performance $[12,37,35,34]$. 
All metrics introduced for NLOS identification in UWB systems can be obtained from a snapshot of the received multi-path signal. In other words, no statistics information over time (variance, mean, pdf and so on) needs to be collected. Therefore, the NLOS identification process speed is very fast.

The pdfs of these metrics are required for likelihood ratio tests. In some cases these pdfs are unavailable. In these scenarios, self-learning techniques used in classification problems can be applied. Examples are support vector machine [38] and neural network [35]. In those methods, a training set is needed, which is a group of channel characteristics data with known LOS/NLOS conditions. Then, the pattern of LOS data and NLOS data can be learned from the training set and the recognized pattern is used to identify NLOS scenario.

A summary of NLOS identification based on channel characteristics and their performance are tabulated in Table 2.2. The identification probability can be one of the followings: the correct decision probability under LOS defined as $P(L O S \mid L O S)$, and the correct decision probability under NLOS defined as $P(N L O S \mid N L O S)$, the overall correct decision probability defined as $P(L O S) P(L O S \mid L O S)+P(N L O S) P(N L O S \mid N L O S)$, where $P(L O S)$ and $P(N L O S)$ are a priori probabilities of respective LOS and NLOS scenarios. In general, the performance order is self-learning techniques $>$ combined metrics $>$ single metric. 
Table 2.2: A summary on channel characteristics based methods

\begin{tabular}{|c|c|c|c|c|}
\hline $\begin{array}{l}\text { Channel charac- } \\
\text { teristics }\end{array}$ & LOS & NLOS & $\begin{array}{l}\text { Identi- } \\
\text { fication } \\
\text { proba- } \\
\text { bility }\end{array}$ & Application/Note \\
\hline $\begin{array}{l}\text { First arriving path } \\
\text { power pdf }\end{array}$ & Rician & Rayleigh & N/A & $\begin{array}{l}\text { Narrow and wide } \\
\text { band, }[28,15]\end{array}$ \\
\hline Rician K factor & High & Low & $\begin{array}{l}71.6- \\
80.0 \%\end{array}$ & $\begin{array}{l}\text { Narrow and wide } \\
\text { band[15, 30, 39] }\end{array}$ \\
\hline $\begin{array}{l}\text { Multipath auto- } \\
\text { correlation }\end{array}$ & High & Low & N/A & $\begin{array}{l}\text { Wide band [31, } \\
16]\end{array}$ \\
\hline $\begin{array}{l}\text { Confidence met- } \\
\text { ric }\end{array}$ & High & Low & $\begin{array}{l}93- \\
100 \%\end{array}$ & $\begin{array}{l}\text { UWB, NDDP de- } \\
\text { tected as NLOS, } \\
{[26]}\end{array}$ \\
\hline $\begin{array}{l}\text { First path power } \\
\left|\alpha_{1}\right|^{2} \& \text { delay of } \\
\text { strongest path } \\
\tau_{\max }-\tau_{1}\end{array}$ & $\begin{array}{l}\text { Large }\left|\alpha_{1}\right|^{2} \quad \& \\
\text { small } \tau_{\max }-\tau_{1}\end{array}$ & $\begin{array}{l}\text { Small }\left|\alpha_{1}\right|^{2} \quad \& \\
\text { large } \tau_{\max }-\tau_{1}\end{array}$ & $\begin{array}{l}87.3- \\
93.6 \%\end{array}$ & $\begin{array}{l}\text { UWB, NDDP de- } \\
\text { tected as NLOS, } \\
\text { [33] }\end{array}$ \\
\hline Change of power & $\begin{array}{l}\text { Increases from } \\
\text { NLOS to LOS }\end{array}$ & $\begin{array}{l}\text { Decreases from } \\
\text { LOS to NLOS }\end{array}$ & $\begin{array}{l}60.3- \\
100 \%\end{array}$ & $\begin{array}{l}\text { UWB, only de- } \\
\text { tect transition, } \\
{[26]}\end{array}$ \\
\hline RSS & High & Low & $78.30 \%$ & UWB, [34, 35] \\
\hline Delay spread & Small & Large & $\begin{array}{l}61.7- \\
100 \% \\
\end{array}$ & $\begin{array}{l}\text { UWB, }[34,12, \\
26]\end{array}$ \\
\hline $\begin{array}{l}\text { Mean excess de- } \\
\text { lay }\end{array}$ & Small & Large & $\begin{array}{l}74.3- \\
100 \%\end{array}$ & UWB, [12] \\
\hline Kurtosis & High & low & $\begin{array}{l}66.3- \\
98.4 \%\end{array}$ & UWB, $[12,36]$ \\
\hline Skewness & High & Low & N/A & UWB, [37] \\
\hline $\begin{array}{l}\text { Combined met- } \\
\text { rics }\end{array}$ & & & $\begin{array}{l}81.8- \\
99.9 \% \\
\end{array}$ & $\begin{array}{l}\text { UWB, }[12,37, \\
35,34]\end{array}$ \\
\hline $\begin{array}{l}\text { Self-learning } \\
\text { techniques }\end{array}$ & & & $91-92 \%$ & UWB. [38, 35] \\
\hline
\end{tabular}

\subsection{Hybrid approaches}

In this section, we introduce hybrid NLOS identification techniques. For either LOS or

NLOS condition, a relationship can be maintained across different channel metrics. For 
example in LOS condition, as the TOA increases, it is expected that the RSS decreases following LOS path loss model. Here, the consistency between different channel metrics is explored to perform NLOS identification.

TOA and RSS matching techniques are discussed in [28, 40, 41]. The intuition behind this method is that if the measured TOA is for a LOS/NLOS BN, then the received power should obey the LOS/NLOS propagation channel model. In [28], the received power is computed from the LOS and NLOS Walfisch-Ikegami path loss model where the distance is substituted with the measured range. Then, the computed power is compared with the measured power (RSS) to see whether it is closer to the LOS model or to the NLOS model.

In [40], the likelihood ratio is given as

$$
\frac{f\left(\hat{L}_{p} \mid \hat{d}, H_{n}\right)}{f\left(\hat{L}_{p} \mid \hat{d}, H_{l}\right)} \stackrel{H_{n}}{\gtrless} \kappa
$$

where $\hat{L}_{p}$ is the estimated path loss, $\hat{d}$ is the estimated range, $H_{n}$ and $H_{l}$ are the respective hypotheses of NLOS and LOS, and $\kappa$ is the threshold that depends on the preassigned false alarm probability.

Three kinds of channel link conditions can be identified in [41]. The likelihood ratio is performed on the conditional pdfs $f\left(C_{i} \mid \hat{d}, \hat{L}_{p}\right), i=0,1,2$, where $C_{0}, C_{1}, C_{2}$ refer to the channel 


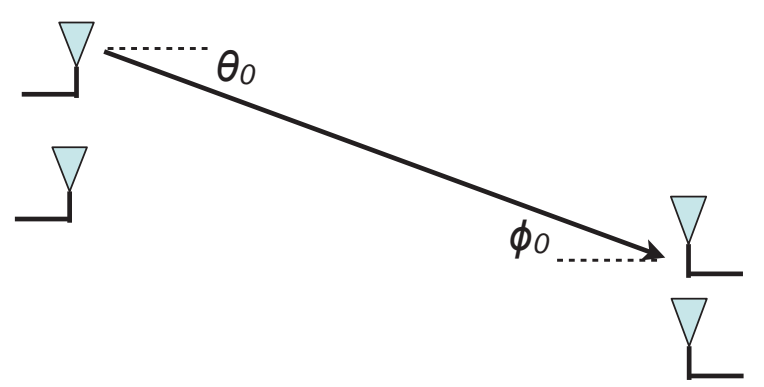

Figure 2.2: The relationship of DOD and DOA of LOS component

link condition: LOS, NDDP, NLOS respectively.

When both sides of the communication channel are equipped with an antenna array, DOA and DOD matching method can be used to identify NLOS [40]. This method is based on an observation of LOS component as illustrated in Figure 2.2: $\theta_{0}=\phi_{0}$. This relationship does not hold for NLOS components.

\subsection{Comparison of NLOS identification methods}

Table 2.3 compares different NLOS identification methods in terms of hardware complex-

ity, software complexity, SNR requirement, processing time and performance. When the antenna array is required, the hardware complexity is considered medium to high. When the DOA or TOA needs to be estimated, the SNR should be high. When the statistics information needs to be collected or the algorithm is complex, it needs a longer processing time. It is observed that channel characteristics-based and antenna array-based methods maintain a good trade-off between requirements and performance: fair to good performance may be achieved with low to medium requirements. 
Table 2.3: Comparison of NLOS identification methods

\begin{tabular}{|l|l|l|l|l|l|}
\hline & $\begin{array}{l}\text { Hardware } \\
\text { complexity }\end{array}$ & $\begin{array}{l}\text { Software } \\
\text { com- } \\
\text { plexity }\end{array}$ & $\begin{array}{l}\text { SNR } \\
\text { require- } \\
\text { ment }\end{array}$ & $\begin{array}{l}\text { Processing } \\
\text { time }\end{array}$ & Performance \\
\hline $\begin{array}{l}\text { Cooperative } \\
\text { methods }\end{array}$ & $\begin{array}{l}\text { Low- } \\
\text { medium }\end{array}$ & High & High & $\begin{array}{l}\text { Medium- } \\
\text { high }\end{array}$ & $\begin{array}{l}\text { Good for } \geq 4 \\
\text { LOS BNs }\end{array}$ \\
\hline $\begin{array}{l}\text { Range statistics } \\
\text { based }\end{array}$ & Low & Low & High & High & Fair \\
\hline $\begin{array}{l}\text { Channel charac- } \\
\text { teristics based }\end{array}$ & Low & $\begin{array}{l}\text { Low- } \\
\text { medium }\end{array}$ & $\begin{array}{l}\text { Low- } \\
\text { medium }\end{array}$ & $\begin{array}{l}\text { Low- } \\
\text { medium }\end{array}$ & Varying \\
\hline Hybrid methods & $\begin{array}{l}\text { Low fhigh for } \\
\text { DOD\&DOA } \\
\text { matching) }\end{array}$ & Low & High & Low & Fair-Good \\
\hline
\end{tabular}

\subsection{Conclusion}

This chapter reviews NLOS identification techniques in the literature. There are a variety of NLOS identification methods with different complexity and performance levels. The cooperative NLOS identification techniques performs well only when there are enough LOS measurements, and the software complexity is high. The range statistics based methods require a high SNR for TOA estimation and also require a long processing time to acquire statistics. Moreover, they may fail to correctly identify NLOS when base nodes, the target node and the scatterers are all stationary. The channel characteristics based and hybrid methods provide a good trade-off between requirements and performance. 


\section{Chapter 3}

\section{NLOS Identification Using Spatial Correlation}

A novel approach for modeling multi-user multi-input and multi-output (MIMO) channels

is proposed. Here, the scatterers are modeled as random rough surfaces: Any point on rough surfaces scatters the incident wave into any given direction with certain probability. This leads to correlation across different spatially distributed users.

The closed form expressions for the intra-user (point-to-point) and inter-user (multi-user) correlation of channel coefficients across antenna elements are derived. It is observed that the distance of users relative to the scatterer surfaces as well as the degree of roughness impact the correlation region. The technique is applied to both non-line-of-sight (NLOS) and line-of-sight (LOS) scenarios. The spatial correlation of channel coefficients across antenna elements is proposed as a metric for NLOS identification. 


\subsection{Introduction}

Multiple input and multiple output (MIMO) wireless communication, featured by multiple antenna elements at the transmitter and receiver, has the potential to increase throughput capacity in rich multipath environments, without increasing transmit power and bandwidth $[42,43]$. Here, the performance is enhanced by the spatial properties of the multipath channel, which is characterized by MIMO channel impulse response matrix. Therefore, MIMO propagation channel modeling is an important prerequisite for evaluating the performance improvements of MIMO systems, such as capacity [42], diversity gain and multiplexing gain [44]. In addition, it is crucial for designing and evaluating space-time coding techniques [45] and transceiver architecture [46] for such systems.

In early MIMO research, a rich scattering environment is assumed, leading to a circularly symmetric complex Gaussian distributed MIMO channel with independent fadings across different antenna pairs $[42,45]$. Due to the analytical feasibility, this statistical model is quite often used. However, the assumption of independent fading across antenna pairs does not always hold in real scenarios where a rich scattering environment may not be available. Spatial correlation of one-ring model is first investigated by Jakes [47]. Moreover, [48] first employs the one-ring model to study the fading correlations in MIMO systems. In addition, the effect of spatially correlated fading on MIMO capacity is analyzed in [48] and [49].

Recently, various spatially correlated MIMO channel models have been studied to describe 
the statistics of the channel matrix. These models include Kronecker product form (KPF) [50], virtual channel representation (VCR) [51] and W-model [52]. The applications of all those models are subject to restrictions: W-model requires the eigenbasis of the transmitter and receiver to be separable [53]; VCR is suitable for large dimension linear arrays; KPF is applicable to environments where both the transmitter and receiver are only surrounded by local scatterers [54]. Another approach in MIMO channel modeling is to describe the properties of the physical multipath propagation channel. Such models are summarized in [55] and an easy-to-use spatio-temporal correlation function of MIMO channel is presented in [56].

Similarly, in recent studies, the channel across different users in multi-user scenarios has been assumed independent. The correlation across the channel of multiple users impacts the sum transmission rate and accordingly the design of transmission schemes. For example, transmission schemes for MIMO broadcast systems with partial channel information has been studied in [57]. We have not encountered a topic on correlated multi-user MIMO channel modeling in articles of this research area. In addition, there is a lack of correlated multi-user models; none of the introduced single user MIMO channel models can be conveniently extended to correlated multi-user scenarios.

Here, we propose a novel approach for modeling multi-user MIMO channels. The technique enables us to study the channel correlation across antenna elements within one user and across users. This is accomplished by characterizing the key features of physical en- 
vironments, which include the geometry and roughness of scattering surfaces. Here, we model the scatterers as a random rough surface with its height being a Gaussian process. Accordingly, any point of the rough surface scatters the incident wave into various directions with the certain probability. This leads to the correlation of signals across multiple users. An example of the relevant scenarios are users located within a valley (e.g., hilly area). In addition, under certain conditions, scatterers located in streets might be similarly modeled.

Technically, Kirchhoff theory [58, Ch.3] is used to calculate the statistics of scattered fields, which is also known as tangent plane or physical optics theory. It is the most widely used theory in the study of wave scattering from rough surfaces: It gives relatively simple analytical expressions for scattered field amplitudes, being readily compared with the experiment results.

In this chapter, the intra-user and cross-user correlation is derived based on Kirchhoff theory. It studies the impact of the incident angle, the distance of the transmitter and the receiver, the surface roughness, and the ratio of the power of the line-of-sight (LOS) and non-LOS (NLOS) on the correlation. The results confirm that: (a) Higher roughness leads to lower correlation; (b) topography plays the main role in the shape of the correlation; (c) more signal power spreads out for larger roughness; (d) the roughness impact would be lower when the incident angle gets smaller; and (e) LOS signals drastically boosts the correlation. . 


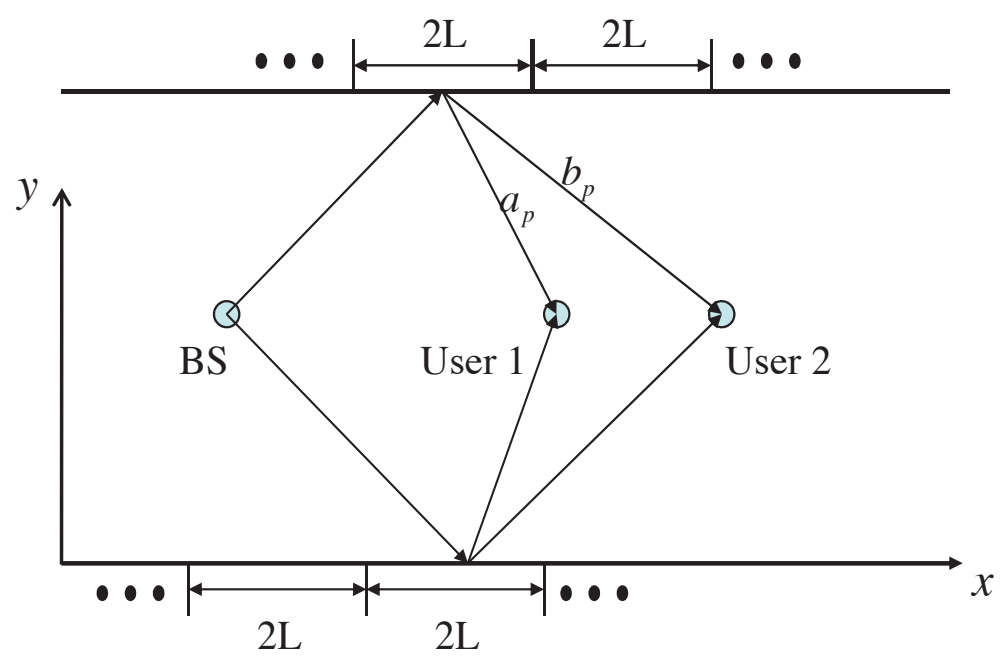

Figure 3.1: Two-user channel for the scattering environment within a valley.

The remainder of the chapter is organized as follows: Section 3.2 presents the multipath two-user channel model and the definitions of LOS and NLOS channel coefficients. Moreover, the proposed single-user MIMO model is compared with some widely accepted MIMO channel models [50]-[52]; Section 3.3 derives a closed form for the intra- and interuser spatial correlation functions; Section 3.4 presents the numerical evaluation of the correlation; Section 3.5 concludes this chapter.

\section{2 multipath two-user MIMO channel matrix}

As shown in Figure 3.1, we consider a simple multi-user broadcast scenario where one base station (BS) deployed with $N$ antennas is capable of transmitting symbols simultaneously to User 1 and User 2, both equipped with $M$ antennas. 


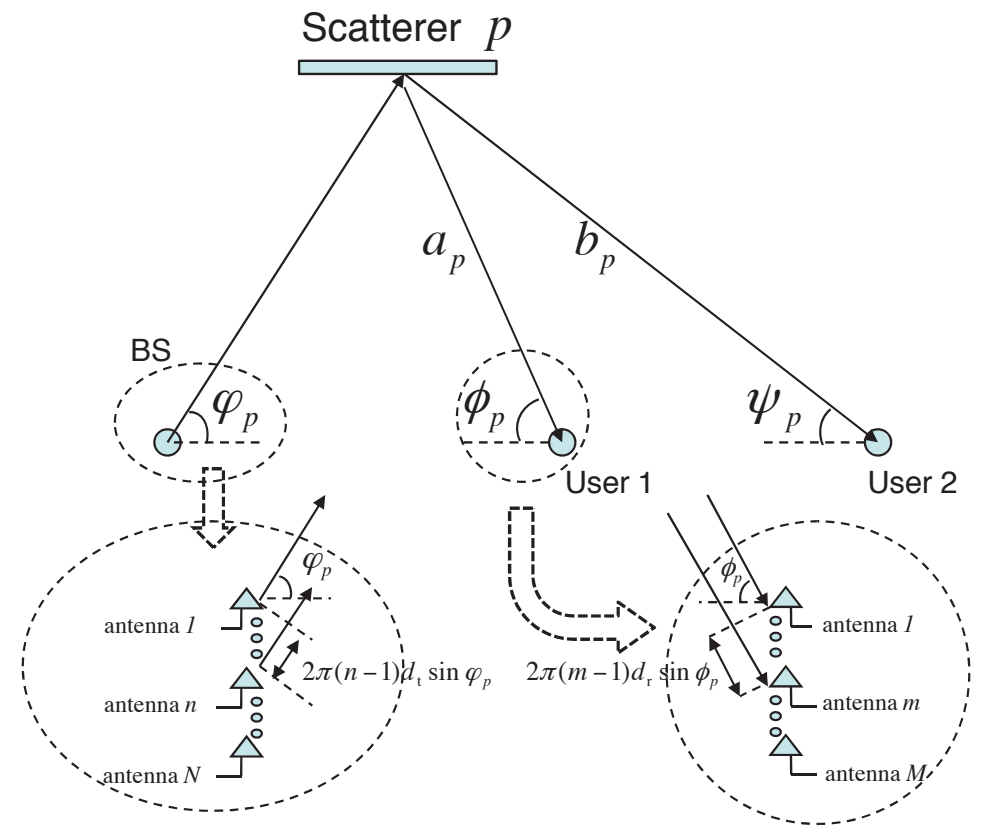

Figure 3.2: Signal arrivals and departures for multi-user MIMO channel.

\subsubsection{NLOS and LOS channel matrices}

Figure 3.1 represents the scattering environment encountered by users located within a valley that is formed by two rough scattering planes represented by the top and the bottom lines. The two scattering planes are partitioned into $P$ segments of length $2 L$ and single bounce reflection is assumed.

In the remainder of this chapter, "scatterer" refers to a segment of a rough scattering plane. We also assume two users are closely located. Thus, the scatterers contributing to the transmission paths between BS and User 1 will contribute to those between BS and User 2 and vice versa. In other words, the scatterers "seen" by User 1 and BS are the same as those "seen" by User 2 and BS. In addition, we assume any point of the rough surface scatters 
the incident energy toward any given direction with certain probability.

In the derivations, we assume the symbol duration is much larger than the delay incurred by different scatterers, i.e., flat fading. Developed from the double-directional propagation model [59], the channel matrix for User $1, \mathbf{H}_{1}^{\mathrm{NLOS}}$, for a NLOS scenario, corresponds to

$$
\begin{aligned}
\mathbf{H}_{1}^{\mathrm{NLOS}} & =\left(\mathbf{e}_{r}\left(\phi_{1}\right) \ldots \mathbf{e}_{r}\left(\phi_{P}\right)\right)\left(\begin{array}{ccc}
a_{1} & \cdots & 0 \\
\vdots & \ddots & \vdots \\
0 & \cdots & a_{P}
\end{array}\right)\left(\begin{array}{c}
\mathbf{e}_{t}^{T}\left(\varphi_{1}\right) \\
\vdots \\
\mathbf{e}_{t}^{T}\left(\varphi_{P}\right)
\end{array}\right) \\
& =\sum_{p=1}^{P} a_{p} \mathbf{e}_{r}\left(\phi_{p}\right) \mathbf{e}_{t}^{T}\left(\varphi_{p}\right)
\end{aligned}
$$

where the scatterer or transmission path denoted by $p$ has a coefficient of $a_{p}$, makes an angle of $\phi_{p}$ with the receive antenna array, and an angle of $\varphi_{p}$ with the transmit antenna array. A detailed scattering geometry of one segment (scatterer $p$ ) is sketched in Figure 3.2. When a signal departs from or arrives at an antenna array, the signal phase at Antenna 1 is taken as reference and at the remaining antennas additional phases are incurred due to relative delay. In (3.2), $\mathbf{e}_{r}\left(\phi_{p}\right)$ and $\mathbf{e}_{r}\left(\varphi_{p}\right)$ are array vectors consisting of such phase shifts. Assuming uniform linear arrays and all antenna arrays are perpendicular to the scatterer surface as shown in Figure 3.2, $\mathbf{e}_{r}\left(\phi_{p}\right)=\left(1, \exp \left(-j 2 \pi d_{r} \sin \phi_{p}\right), \ldots, \exp \left(-j 2 \pi(M-1) d_{r} \sin \phi_{p}\right)\right)^{T}$, $\mathbf{e}_{t}\left(\varphi_{p}\right)=\left(1, \exp \left(-j 2 \pi d_{t} \sin \varphi_{p}\right), \ldots, \exp \left(-j 2 \pi(N-1) d_{t} \sin \varphi_{p}\right)\right)^{T}$ where $d_{t}$ and $d_{r}$ are the receive and transmit antenna separation normalized to wavelength $\lambda$, respectively.

The antennas radiation pattern and polarization may be considered in the channel model 
(3.2). The radiation pattern for each antenna element can be characterized by the azimuth gain $G_{r}\left(\phi_{p}\right)$ and $G_{t}\left(\varphi_{p}\right)$ for the receiver and transmitter, respectively. Therefore the channel formulation (3.2) can be rewritten as

$$
\mathbf{H}_{1}^{\mathrm{NLOS}}=\sum_{p=1}^{P} a_{p} G_{r}\left(\phi_{p}\right) G_{t}\left(\varphi_{p}\right) \mathbf{e}_{r}\left(\phi_{p}\right) \mathbf{e}_{t}^{T}\left(\varphi_{p}\right)
$$

It is expected that differing polarization between the incoming waves and the receive antennas reduces the received power. In the derivation of (3.30), we assumed linearly polarized incident wave for the transmit antennas. This has been underlined in the assumptions presented after (3.32). If the incident wave is vertically or horizontally polarized, it is reasonable to consider the polarization of the scattered field unchanged and there is no power reduction at a receiver whose antenna has the same linear polarization [58, Ch.8] However, if the polarization of the scattered field is changed, a polarization mismatch loss will be introduced on $a_{p}$ in (3.2), which in turn impacts the channel correlation.

Similarly, a channel matrix for User 2 corresponds to

$$
\mathbf{H}_{2}^{\mathrm{NLOS}}=\sum_{p=1}^{P} b_{p} \mathbf{e}_{r}\left(\psi_{p}\right) \mathbf{e}_{t}^{T}\left(\varphi_{p}\right)
$$

where the angle of departure (AOD) characterized by the left array vectors remains the same as in (3.2), the signal directed to User 1 with angle of arrival (AOA) $\phi_{p}$ reaches User 
2 at a new angle $\psi_{p}$, determined by the location information of User 2 relative to User 1 and the scatterer. Note that $\mathbf{H}_{1}$ in (3.2) differs from $\mathbf{H}_{2}$ in (3.4) in the angle of arrival $\left(\phi_{p}\right.$ and $\left.\psi_{p}\right)$ and associated path coefficient $\left(a_{p}\right.$ and $\left.b_{p}\right)$.

Using (3.2), the channel coefficients from the BS's Antenna $n$ to the User 1's Antenna $m$ for NLOS and LOS are:

$$
\begin{aligned}
h_{m n}^{(1) \mathrm{NLOS}} & =\sum_{p=1}^{P} a_{p} \exp \left(-j 2 \pi\left(d_{r}(m-1) \sin \phi_{p}\right.\right. \\
& \left.\left.+d_{t}(n-1) \sin \varphi_{p}\right)\right) \\
h_{m n}^{(1) \mathrm{LOS}} & =a_{0}+h_{m n}^{(1) \mathrm{NLOS}}
\end{aligned}
$$

where the scatterer or transmission path $p, p \in\{1,2, \ldots, P\}$, has the coefficient of $a_{p}$, which is a complex random variable. In addition, $a_{0}$ represents the LOS component and:

$$
a_{0}=\sqrt{K_{1} \Omega_{1}} e^{-j 2 \pi / \lambda d_{1}}
$$

Here $\Omega_{1}=E\left(\left|h_{m n}^{(1) \mathrm{NLOS}}\right|^{2}\right)(\mathrm{E}(\cdot)$ denotes statistical expectation and $|\cdot|$ represents absolute value), $K_{1}$ is the Rice factor, defined as the ratio of the direct path power to the diffuse component power, i.e., $K_{1}=\left|a_{0}\right|^{2} / \Omega_{1}, \lambda$ is the wavelength, and $D_{1}$ denotes the distance between BS and User 1.

The channel coefficients from BS Antenna s to User 2's Antenna $t$ can be similarly defined, 
with $b_{0}$ representing the LOS component and $K_{2}$ representing the Rice factor of User 2 .

Uncorrelated scatterers coefficients are assumed, i.e.,

$$
\begin{aligned}
& \mathrm{E}\left(a_{i} a_{j}^{*}\right)=0, \quad \mathrm{E}\left(b_{i} b_{j}^{*}\right)=0, \quad \mathrm{E}\left(a_{i} b_{j}^{*}\right)=0, \\
& \text { if } i \neq j \quad i, j=1,2, \ldots, P
\end{aligned}
$$

where $*$ denotes conjugate. These hold because the surface profiles of different segments are uncorrelated (see (3.22) and the assumption of $L \gg T$ before (3.33)).

Let us define the normalized spatial correlations between the channel coefficients across users (inter-user) and within one user (intra-user):

Inter-user

$$
\begin{gathered}
\gamma_{m n, s t}^{(1)(2) \mathrm{NLOS}}=E\left(h_{m n}^{(1) \mathrm{NLOS}} h_{s t}^{(2) \mathrm{NLOS} *}\right) / E\left(\left|h_{m n}^{(1) \operatorname{LOS}}\right|^{2}\right) \\
\gamma_{m n, s t}^{(1)(2) \operatorname{LOS}}=E\left(h_{m n}^{(1) \operatorname{LOS}} h_{s t}^{(2) \operatorname{LOS} *}\right) / E\left(\left|h_{m n}^{(1) \operatorname{LOS}}\right|^{2}\right)
\end{gathered}
$$

Intra-user

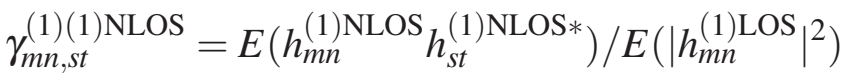

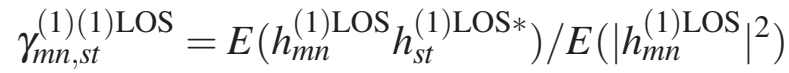


where the normalization factor $E\left(\left|h_{m n}^{(1) \operatorname{LOS}}\right|^{2}\right)$ is selected as the maximum correlation which happens when User 2 coincides with User 1 in LOS scenarios. Therefore, the generated normalized correlation lies within the interval $[0,1]$.

Assuming uncorrelated scattering, the spatial correlations would correspond to:

$$
\begin{aligned}
\mathrm{E}\left(h_{m n}^{(1) \mathrm{NLOS}} h_{s t}^{(2) \mathrm{NLOS} *}\right) & =\sum_{p=1}^{P} \mathrm{E}\left(a_{p} b_{p}^{*}\right) \exp \left(-j 2 \pi\left(d _ { r } \left((m-1) \sin \phi_{p}\right.\right.\right. \\
\left.\left.\left.-(s-1) \sin \psi_{p}\right)+d_{t}(n-t) \sin \varphi_{p}\right)\right) & \\
\mathrm{E}\left(h_{m n}^{(1) \mathrm{NLOS}_{h}(1) \mathrm{NLOS} *}\right) & =\sum_{p=1}^{P} \mathrm{E}\left(a_{p} a_{p}^{*}\right) \exp \left(-j 2 \pi\left(d_{r}\left((m-s) \sin \phi_{p}\right)\right.\right. \\
& \left.\left.+d_{t}(n-t) \sin \varphi_{p}\right)\right)
\end{aligned}
$$

In order to evaluate the intra-user and inter-user correlations, $\mathrm{E}\left(a_{p} b_{p}^{*}\right)$ and $\mathrm{E}\left(a_{p} a_{p}^{*}\right)$ are evaluated in Section 3.3.

\subsubsection{Comparison with other channel models}

In this section, the benefits and limitations of the proposed model are discussed and compared with respect to some widely accepted single-user MIMO models, i.e., Kronecker product form (KPF), virtual channel representation (VCR) and W-model. First, the basis 
of those models is introduced. The KPF model, which is reported in [50], assumes separate correlation at the transmitter and the receiver. With $N$ transmit antennas and $M$ receive antennas, the channel matrix is expressed as

$$
\mathbf{H}=\mathbf{G}_{R X}^{\frac{1}{2}} \mathbf{H}_{\mathrm{i} . \mathrm{i} . \mathrm{d}} \mathbf{G}_{T X}^{\frac{1}{2} T}
$$

where $\mathbf{H}_{\text {i.i.d }}$ is an $M \times N$ matrix consisting of identical independent zero-mean complex Gaussian elements, $\mathbf{G}_{R X}$ and $\mathbf{G}_{T X}$ are receive and transmit correlation matrices respectively, and $(\cdot)^{1 / 2}$ denotes the matrix square root such that $\mathbf{G}_{R X}^{1 / 2}\left(\mathbf{G}_{R X}^{1 / 2}\right)^{H}=\mathbf{G}_{R X}$. The assumption of separate correlation is valid only when scatterers are local to either transmitters or receivers. Such a scenario is usually applicable in the traditional cellular networks where the scatterer distributions follow one ring [48] or two-ring model [60]. The former has one ring of scatterers around the mobile stations and the latter has one ring around the transmitter and another around the receiver. This limitation is due to the fact that KPF is a special case of VCR [61]. The KPF model can be both simulated and analyzed with great mathematical simplicity; therefore, it is widely used in industrial standards, such as IEEE 802.11n.

VCR model is proposed in [51] and has its roots in double-directional propagation model [59]. The formulation of VCR is

$$
\mathbf{H}=\mathbf{A}_{R X} \mathbf{H}_{V} \mathbf{A}_{T X}^{H}
$$


where $\mathbf{A}_{R X}$ and $\mathbf{A}_{T X}$ are discrete Fourier transform (DFT) matrices which describe the angular directions of scatterers at the receiver and transmitter. In addition, the entries of $\mathbf{H}_{V}$ are independent zero-mean Gaussian distributed. $\mathbf{H}$ and $\mathbf{H}_{V}$ can be viewed as a two dimensional Fourier transform pair. $\mathbf{H}_{V}$ captures the essence of the scattering environment, and reveals two factors effecting the capacity: the number of parallel channels and the level of diversity [51]. The limitation of this model lies in the fact that the scatterer clusters corresponding to the elements of $\mathbf{H}_{V}$ may not distinct from each other, which results in correlation between the elements of $\mathbf{H}_{V}$. This is due to the fact that the angular directions of scatters are not necessarily aligned with the predefined DFT directions. In order to decouple the elements of $\mathbf{H}_{V}$, large arrays which can increase angle resolution have to be employed.

$\mathrm{W}$-model is similar to VCR model. However, W-model lifts the restriction on angular direction, which makes it applicable in versatile environments. The channel matrix is

$$
\mathbf{H}=\mathbf{U}_{R X} \mathbf{H}_{W} \mathbf{U}_{T X}^{H}
$$

where $\mathbf{U}_{R X}, \mathbf{U}_{T X}$ are the unitary spatial eigenbasis at the receiver and the transmitter, respectively, and $\mathbf{H}_{W}$ is independent zero-mean Gaussian distributed. According to [62], the structure defined by (3.17) implies that the transmit antennas are clustered together and the receive antennas are clustered together. In addition, the distance between the two antenna arrays is greatly larger than the array size. Thus the channels of distributed MIMO systems 
where the transmit antennas and/or receive antennas are not clustered together, cannot be described by W-model.

The proposed rough surface model is indeed a double-directional propagation model. Equation (3.1) can be represented as

$$
\mathbf{H}_{1}=\mathbf{E}_{R X} \mathbf{H}_{D} \mathbf{E}_{T X}^{T}
$$

where $\mathbf{E}_{R X}$ denotes the receive directions, $\mathbf{E}_{T X}$ denotes the transmit directions, and $\mathbf{H}_{D}$ is a diagonal matrix. Compared to previous models, it has merits in the theoretical computation of channel gains and spatial correlation based on scattering surface profile and extendibility to multi-user channel model. This model is appropriate for environments where the scatterers can be modeled as random rough surfaces. In addition, some parameters describing the surface profile are required, i.e., the length, $L$, of the scattering plane, the correlation distance, $T$, and the standard deviation, $\sigma$, of the rough surface height, which will be introduced in Section 3.3. The suitable environments, benefits and limitations of the four models are summarized in Table 3.1 .

\subsection{Statistics of the scattered field}

In this section, the statistics of the scattered electromagnetic field from a normally distributed random rough scattering surface are investigated. First, $\mathrm{E}\left(a_{p} b_{p}^{*}\right)$, the correlation of 
Table 3.1: Comparison of four MIMO channel models.

\begin{tabular}{|l|l|l|l|}
\hline KPF & $\begin{array}{l}\text { Suitable envi- } \\
\text { ronments }\end{array}$ & Benefits & Limitations \\
\hline only scatterers & $\begin{array}{l}\text { Scatters dis- } \\
\text { tribute mainly } \\
\text { according to pre- } \\
\text { defined angular } \\
\text { directions }\end{array}$ & $\begin{array}{l}\text { Capturing the effects of scat- } \\
\text { tering characteristics on chan- } \\
\text { nel capacity and diversity }\end{array}$ & $\begin{array}{l}\text { Requiring certain scat- } \\
\text { ter distribution or large } \\
\text { arrays }\end{array}$ \\
\hline $\begin{array}{l}\text { W- } \\
\text { model }\end{array}$ & $\begin{array}{l}\text { Versatile environ- } \\
\text { ments }\end{array}$ & $\begin{array}{l}\text { The limitations on VCR be- } \\
\text { ing lifted }\end{array}$ & $\begin{array}{l}\text { Requiring clustered an- } \\
\text { tennas at the TX and } \\
\text { RX }\end{array}$ \\
\hline $\begin{array}{l}\text { Rough } \\
\text { Surface } \\
\text { model }\end{array}$ & $\begin{array}{l}\text { Large plane scat- } \\
\text { terers }\end{array}$ & $\begin{array}{l}\text { theoretically computing the } \\
\text { channel gains and spatial cor- } \\
\text { relation based on scattering } \\
\text { surface profile and extending } \\
\text { to multi-user channel model }\end{array}$ & $\begin{array}{l}\text { Restricted to certain en- } \\
\text { vironments and requir- } \\
\text { ing some parameters of } \\
\text { the scattering surface }\end{array}$ \\
\hline
\end{tabular}

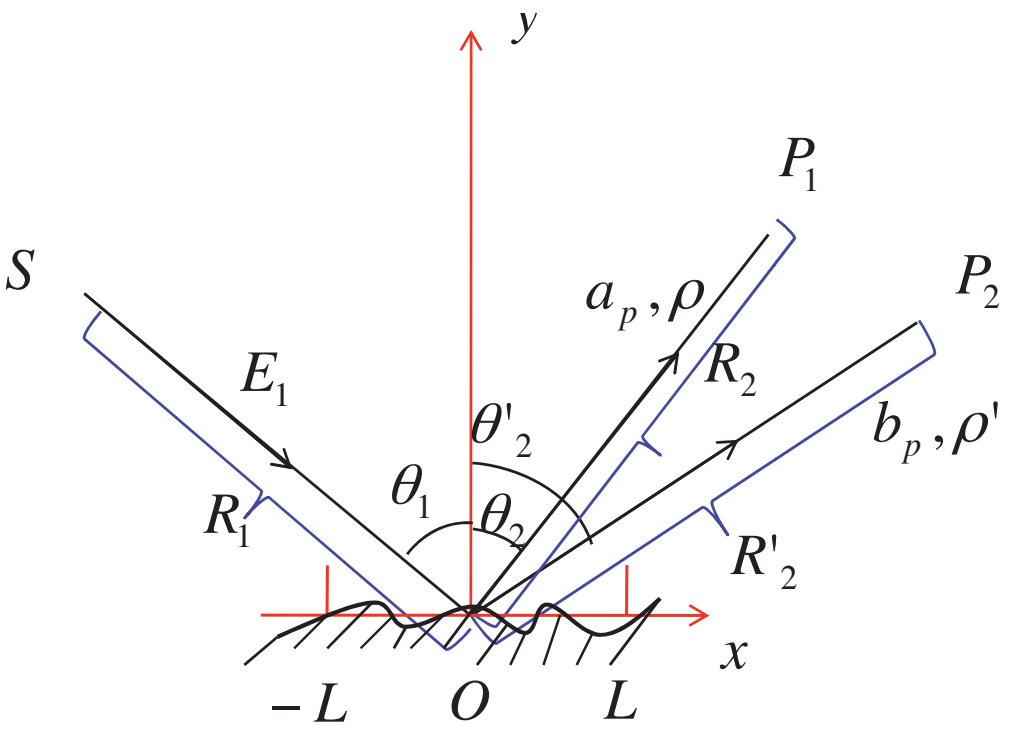

Figure 3.3: The scattering geometry.

the scattered field from scatterer $p$ in different directions $a_{p}$ and $b_{p}$, is computed. Then, the power of the field $\mathrm{E}\left(a_{p} a_{p}^{*}\right)$ is obtained as a particular case of $\mathrm{E}\left(a_{p} b_{p}^{*}\right)$. 
In Figure 3.3, we use Cartesian coordinates $x, y$ to represent the function of a one-dimensional rough scattering plane

$$
\xi=\xi(x)
$$

and the mean level of the surface is the plane

$$
y=0
$$

In (3.19), $\xi$ represents the surface height and is assumed to be Gaussian distributed with mean value 0 and standard deviation $\sigma$. Incorporating Equation (2) in [58, Ch.5.3], its probability density function corresponds to

$$
w(\xi=y)=\frac{1}{\sigma \sqrt{2 \pi}} \exp \left(\frac{-y^{2}}{2 \sigma^{2}}\right) .
$$

The surface height $\xi$ is a stochastic process in space coordinate $x$, and such a process is further assumed to be stationary. Moreover, we assume the autocorrelation coefficient of $\xi(x)$ and $\xi\left(x^{\prime}\right)$ to follow

$$
C(\tau)=e^{-\tau^{2} / T^{2}}, \quad \tau:=x-x^{\prime}
$$

where $T$ is the correlation distance, at which $C(\tau)$ will drop to the value $e^{-1}$. (see Equation (4) in $[58$, Ch.5.3])

As shown in Figure 3.3, a random rough surface runs from $-L$ to $L$. The signal source is denoted by $S$ and the incident field $E_{1}$ is assumed a plane wave and there is no power loss. 
Thus,

$$
E_{1}=e^{-i k R_{1}}, \quad R_{1} \gg L
$$

where $i=\sqrt{-1}, k=2 \pi / \lambda$ is the modulus of wave vector and $R_{1}$ is the distance from the source to the origin (point $O$ in Figure 3.3). Due to the roughness of the surface, in (3.23), the phase is not actually $k R_{1}$. However, it does not impact the derivation as later its effect is cancelled in (3.28). In Figure 3.3, the points $P_{1}$ and $P_{2}$ are in the far field of the scattering plane, i.e., $R_{2}$ and $R_{2}^{\prime}$ are the distance of $P_{1}$ and $P_{2}$ with respect to the origin.

In Figure 3.3, the angle of incidence is denoted by $\theta_{1}$ and the path coefficient or scattered field at $P_{1}$ is denoted by $a_{p}$ with a scattering angle $\theta_{2}$. Moreover, at point $P_{2}$, the scattered field is denoted by $b_{p}$ with a scattering angle of $\theta_{2}^{\prime}$. The angle of incidence and the scattering angle are measured with respect to the $z$ axis. The scattering coefficient $\rho$ corresponding to $a_{p}$ is defined as:

$$
\rho=\frac{a_{p}}{a_{p 0} E_{1}}
$$

where $a_{p 0}$ is the field specularly reflected $\left(\theta_{2}=\theta_{1}\right)$ by a smooth and perfectly conducting plane of the same dimension with the same angle of incident at the same distance when the incident wave is horizontally polarized and $a_{p 0}$ is expressed as

$$
a_{p 0}=\frac{i k e^{-i k R_{2}} L \cos \theta_{1}}{\pi} .
$$

where the parameters are defined in Figure 3.3. 
To take care of the difficulties associated with directly dealing with $a_{p}$, we instead study the statistics of $\rho$. Incorporating (3.23), (3.24), (3.25), the relationship of $\rho$ and $a_{p}$ corresponds to:

$$
a_{p}=\frac{i k e^{-i k\left(R_{1}+R_{2}\right)} L \cos \theta_{1}}{\pi} \rho .
$$

Here, $R_{1}$ and $R_{2}$ are the distances of the transmitter and User 1 from the rough surface respectively (see Figure 3.3). Similarly, $\rho^{\prime}$ (that is the scattering coefficient of $b_{p}$ ) has the following relationship with $b_{p}$ :

$$
b_{p}=\frac{i k e^{-i k\left(R_{1}+R_{2}^{\prime}\right)} L \cos \theta_{1}}{\pi} \rho^{\prime} .
$$

Hence,

$$
\begin{aligned}
& \mathrm{E}\left(a_{p} b_{p}^{*}\right)=\frac{4 e^{-i k\left(R_{2}-R_{2}^{\prime}\right)} L^{2} \cos ^{2} \theta_{1}}{\lambda^{2}} \mathrm{E}\left(\rho\left(\rho^{\prime}\right)^{*}\right) \\
& \mathrm{E}\left(a_{p} a_{p}^{*}\right)=\frac{4 L^{2} \cos ^{2} \theta_{1}}{\lambda^{2}} \mathrm{E}\left(|\rho|^{2}\right)
\end{aligned}
$$

Next, $\mathrm{E}\left(\rho\left(\rho^{\prime}\right)^{*}\right)$ is calculated in order to compute $\mathrm{E}\left(a_{p} b_{p}^{*}\right)$, where $\rho$ is calculated based on the Kirchhoff approximation of the surface conditions which are required to evaluate the Helmholtz integral [58, Ch.3.1].

$$
\rho=\frac{F\left(\theta_{1}, \theta_{2}\right)}{2 L} \int_{-L}^{L} e^{i \mathbf{v}^{\mathrm{T}} \mathbf{r}} \mathrm{d} x
$$


where $\mathbf{r}=(x, \xi(x))^{\mathrm{T}}$, and

$$
\begin{aligned}
& \mathbf{v}=\left(v_{x}, v_{z}\right)^{\mathrm{T}} \\
& =\left(\frac{2 \pi}{\lambda}\left(\sin \theta_{1}-\sin \theta_{2}\right),-\frac{2 \pi}{\lambda}\left(\cos \theta_{1}+\cos \theta_{2}\right)\right)^{\mathrm{T}} \\
& F\left(\theta_{1}, \theta_{2}\right)=\frac{1+\cos \left(\theta_{1}+\theta_{2}\right)}{\cos \theta_{1}\left(\cos \theta_{1}+\cos \theta_{2}\right)}
\end{aligned}
$$

In (3.30), the exponent of the integrand $\mathbf{v}^{\mathrm{T}} \mathbf{r}$ is essentially the phase of the wave at $P_{1}$ contributed by a small part of the surface. Therefore, integrating such contributions at $P_{1}$ along the surface results in (3.30). As noted in (3.30), $\rho$ is the integral of random process, hence it is a random variable.

The derivation of (3.30) is subject to the following assumptions:

1. The incident wave is plane (requiring $R_{1} \gg L$ ) and linearly polarized. This assumption can be withdrawn by conducting an integration over space vectors used to describe the polarization [58, Ch.8].

2. The surface is perfectly conducting. This assumption can be withdrawn by including the relative permeability in the integrant of (3.30).

3. Mutual interaction of the irregularities on rough surface (shadowing and multiple scattering) are neglected. This assumption can be withdrawn by integrating over the illuminated parts of the surface and introducing secondary reflection coefficients for 
multiple scattering.

4. The field at any point of the surface can be approximated by the field that would be present on the tangent plane at that point, which requires that the correlation distance, $T$, defined in (3.22) to be much greater that the wavelength, i.e., $T \gg \lambda$. This assumption is essential in the Kirchhoff approach.

5. To ensure the availability of a rough surface, the dimension of surfaces should be greater than the correlation distance, i.e., $L \gg T$.

6. The receiver is located sufficiently far from the rough surface $\left(R_{2} \gg L\right)$ to ensure the availability of plane scattered waves. This is also can be dropped by considering spherical scattered waves where $\theta_{1}$ and $\theta_{2}$ in (3.31) are dependent on the considered part of the surface, i.e., $x$ in (3.30).

Some of the assumptions can be withdrawn at the cost of mathematical simplicity. Besides, these assumptions do not seriously impair the generality of our solution, because it is a well-known fact that the roughness of a surface modifies the scattered field far more than its electrical properties. 
Then, $E\left(\rho\left(\rho^{\prime}\right)^{*}\right)$ in (3.28) is corresponds to (Appendix-A)

$$
\begin{aligned}
& \mathrm{E}\left(\rho\left(\rho^{\prime}\right)^{*}\right) \approx F\left(\theta_{1}, \theta_{2}\right) F\left(\theta_{1}, \theta_{2}^{\prime}\right)\left(\frac{\sqrt{\pi} T}{2 L} \operatorname{sinc}\left(\left(v_{x}-v_{x}^{\prime}\right) L\right) e^{-g_{1}}\right. \\
& \left.\sum_{m=1}^{\infty} \frac{g_{2}^{m}}{m ! \sqrt{m}} \exp \left(-\frac{v_{x}^{2} T^{2}}{4 m}\right)+e^{-g_{1}} \operatorname{sinc}\left(v_{x} L\right) \operatorname{sinc}\left(v_{x}^{\prime} L\right)\right)
\end{aligned}
$$

where $F\left(\theta_{1}, \theta_{2}\right)$ is defined in (3.32), $\operatorname{sinc}(x)=\sin (x) / x, g_{1}=\frac{1}{2} \sigma^{2}\left(v_{z}^{2}+v_{z}^{\prime 2}\right), g_{2}=v_{z} v_{z}^{\prime} \sigma^{2}$, $v_{z}$ and $v_{x}$ are defined in (3.31), $F\left(\theta_{1}, \theta_{2}^{\prime}\right)$ is obtained similar to $F\left(\theta_{1}, \theta_{2}\right)$ by replacing $\theta_{2}$ with $\theta_{2}^{\prime}$, and $T$ is the corelation distance.

Letting $\theta_{2}^{\prime}=\theta_{2}, \mathrm{E}\left(\rho \rho^{*}\right)$ is readily derived and it agrees with the expression given in [58,

\section{Ch.5.3].}

To analyze the dependence of $\mathrm{E}\left(\rho\left(\rho^{\prime}\right)^{*}\right)$ on $g_{2}, v_{x}, v_{x}^{\prime}$ and $T$, we consider three cases: 1$)$ $\left.\left.g_{2} \ll 1,2\right) g_{2} \approx 1,3\right) g_{2} \gg 1$. Because $g_{2}$ is proportional to $\sigma^{2} / \lambda^{2}$, these three cases correspond to a slightly, moderately and very rough surface.

$g_{2} \ll 1$ : The series in (3.33) converges so quickly that we may only consider its first term. Thus,

$$
\begin{aligned}
& \mathrm{E}\left(\rho\left(\rho^{\prime}\right)^{*}\right) \approx F\left(\theta_{1}, \theta_{2}\right) F\left(\theta_{1}, \theta_{2}^{\prime}\right)\left(\frac{\sqrt{\pi} T}{2 L} \operatorname{sinc}\left(\left(v_{x}-v_{x}^{\prime}\right) L\right)\right. \\
& \left.e^{-g_{1}} g_{2} \exp \left(-\frac{v_{x}^{2} T^{2}}{4}\right)+e^{-g_{1}} \operatorname{sinc}\left(v_{x} L\right) \operatorname{sinc}\left(v_{x}^{\prime} L\right)\right)
\end{aligned}
$$



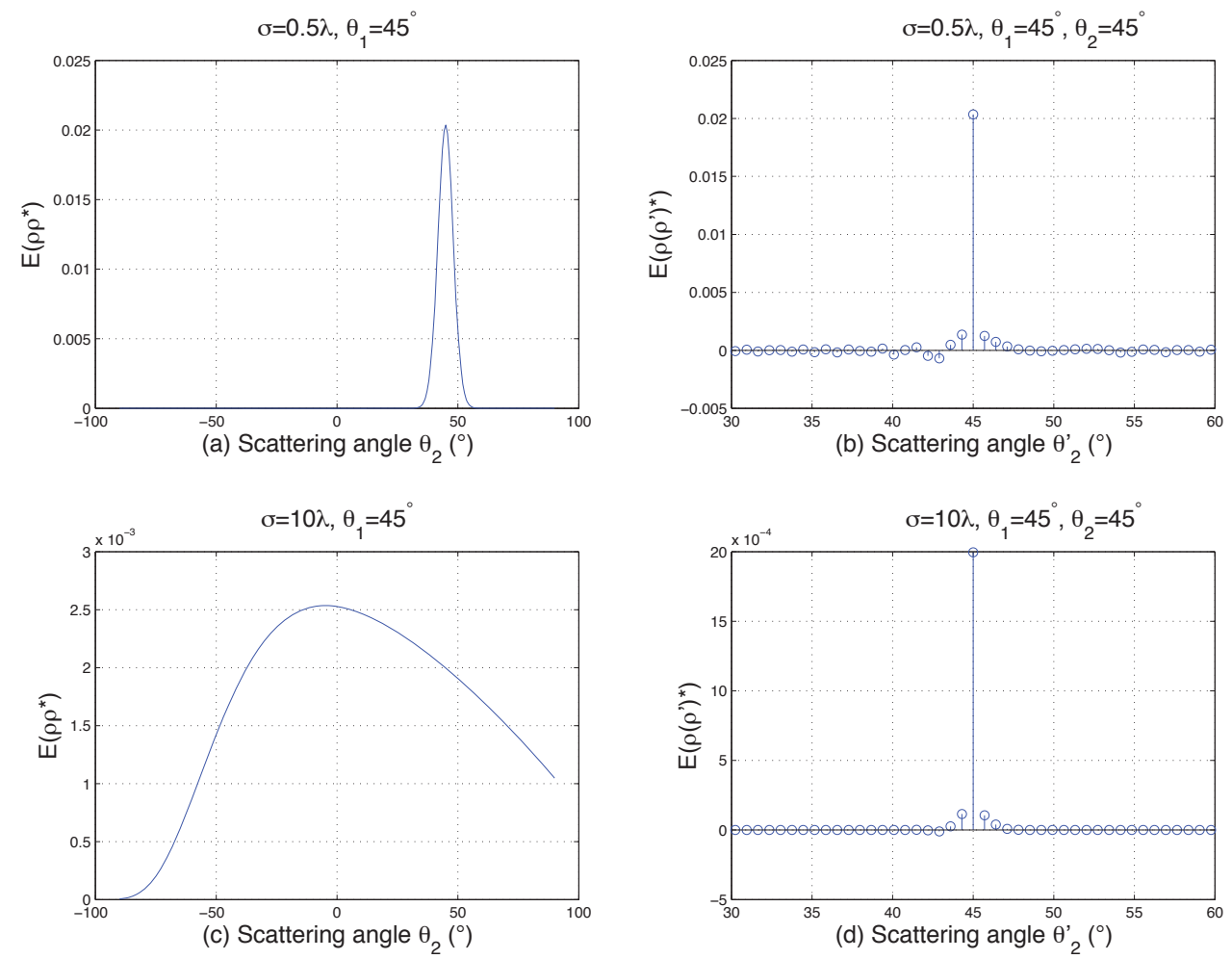

Figure 3.4: Statistics of the scattering coefficients: $\mathrm{E}\left(\rho \rho^{*}\right)$ and $\mathrm{E}\left(\rho\left(\rho^{\prime}\right)^{*}\right)$ for $T=$ $25 \lambda, L=250 \lambda, \theta_{1}=\theta_{2}=45^{\circ}$.

$g_{2} \approx 1$ : As $g_{2}$ grows larger, the series converges slower and an increasing number of terms of the series (3.33) must be taken into consideration.

$g_{2} \gg 1$ : The series in (3.33) converges too slowly to be of any practical use and we use another approach (Appendix-B) to get

$$
\begin{aligned}
& \mathrm{E}\left(\rho\left(\rho^{\prime}\right)^{*}\right) \approx F\left(\theta_{1}, \theta_{2}\right) F\left(\theta_{1}, \theta_{2}^{\prime}\right)\left(\frac{1}{L} \operatorname{sinc}\left(\left(v_{x}-v_{x}^{\prime}\right) L\right) e^{g_{2}-g_{1}}\right. \\
& \left.\sqrt{\frac{\pi}{g_{2}}} T \exp \left(-\frac{v_{x}^{2} T^{2}}{4 g_{2}}\right)+e^{-g_{1}} \operatorname{sinc}\left(v_{x} L\right) \operatorname{sinc}\left(v_{x}^{\prime} L\right)\right)
\end{aligned}
$$



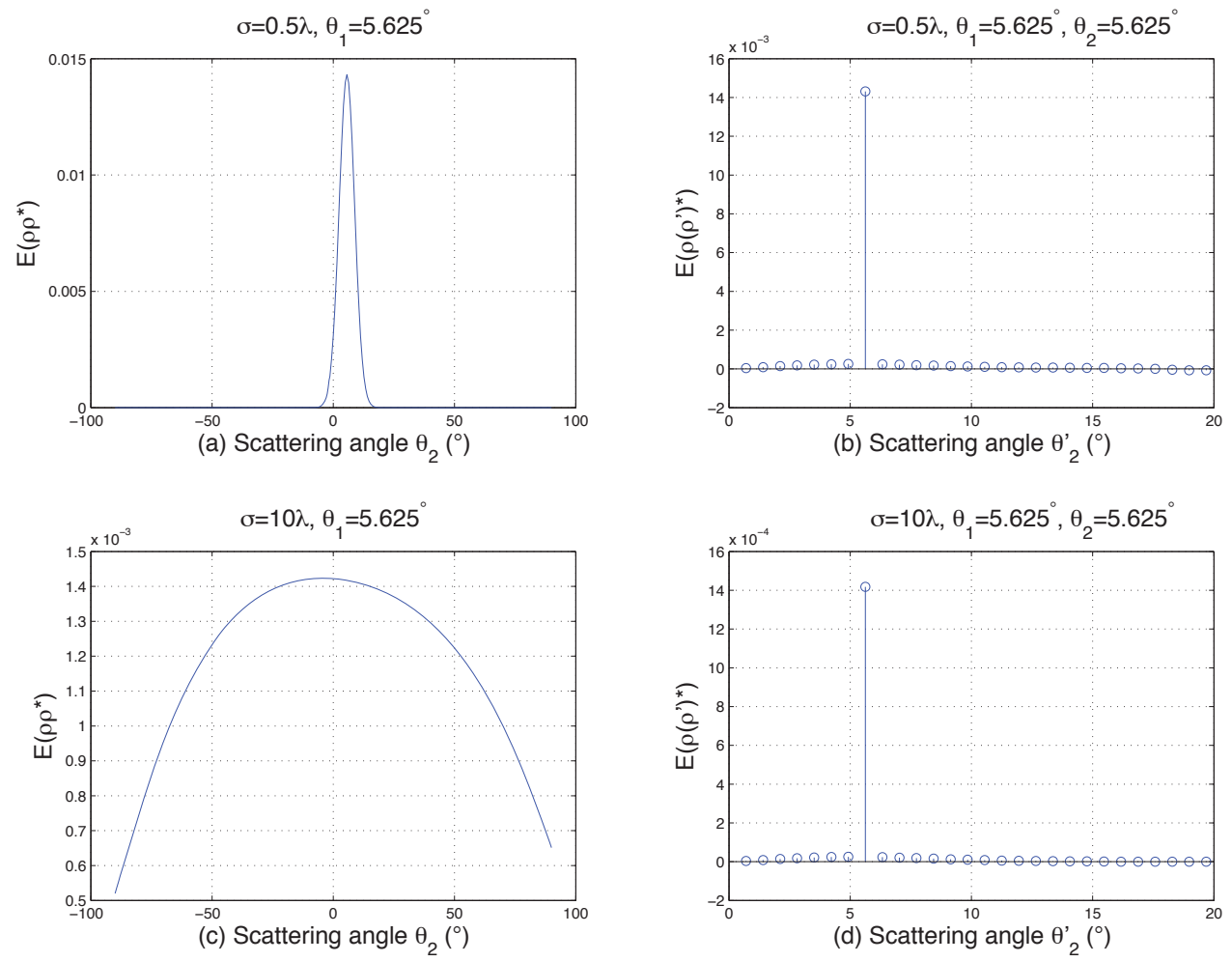

Figure 3.5: Statistics of the scattering coefficients: $\mathrm{E}\left(\rho \rho^{*}\right)$ and $\mathrm{E}\left(\rho\left(\rho^{\prime}\right)^{*}\right)$ for $T=$ $25 \lambda, L=250 \lambda, \theta_{1}=\theta_{2}=5.625^{\circ}$.

Now, we investigate the impact of roughness, and the incident and reflected angles on the reflection coefficient auto and cross correlations. Autocorrelation is a measure of the scattering power, while the cross correlation is a measure of the correlation of scattered waves across two reflected angles. Given $L=250 \lambda, T=25 \lambda, \theta_{1}=45^{\circ}$, the scattering power $\mathrm{E}\left(\rho \rho^{*}\right)$ versus scattering angle $\theta_{2}$ is plotted in Figure 3.4 for $\sigma=0.5 \lambda$ and $\sigma=$ 10ג. The cross correlation $\mathrm{E}\left(\rho\left(\rho^{\prime}\right)^{*}\right)$ versus scattering angle $\theta_{2}^{\prime}$ is also plotted, where $\rho$ has the scattering angle $\theta_{2}=45^{\circ}$. $\mathrm{E}\left(\rho \rho^{*}\right)$ plots in (a) and (c) show that more signal power spreads out as roughness increases. In addition, in (c), the maximum power shifts toward the incident direction. This phenomenon can be explained by a simple geometrical 

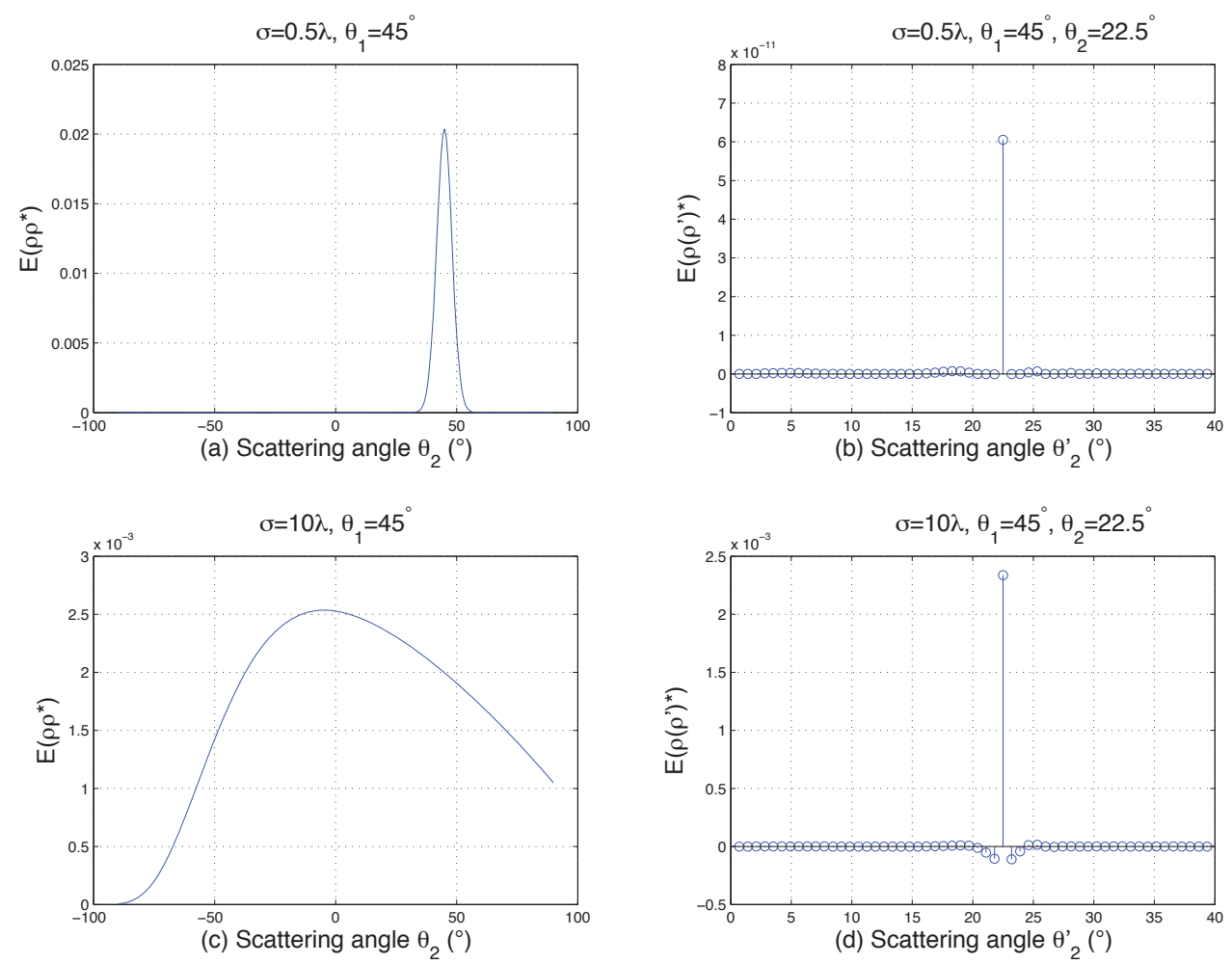

Figure 3.6: Statistics of the scattering coefficients: $\mathrm{E}\left(\rho \rho^{*}\right)$ and $\mathrm{E}\left(\rho\left(\rho^{\prime}\right)^{*}\right)$ for $T=$ $25 \lambda, L=250 \lambda, \theta_{1}=45^{\circ}, \theta_{2}=22.5^{\circ}$.

ray tracing. From $\mathrm{E}\left(\rho\left(\rho^{\prime}\right)^{*}\right)$ plots (b) and (d), we see that, regardless of roughness, the correlation tends to zero very fast when the separation of $\theta_{2}^{\prime}$ and $\theta_{2}$ increases. $\mathrm{E}\left(\rho\left(\rho^{\prime}\right)^{*}\right)$ is significant only when $\theta_{2}^{\prime}$ is within an interval about $2^{\circ}$ around $\theta_{2}$. This can be explained by the fact that the term $\operatorname{sinc}\left(\left(v_{x}-v_{x}^{\prime}\right) L\right)$ dominates (3.33) (the second summand in (3.33) is negligible ) and $v_{x}-v_{x}^{\prime}=\left(\sin \theta_{2}^{\prime}-\sin \theta_{2}\right) 2 \pi / \lambda$. Therefore, when the difference of $\theta_{2}^{\prime}$ and $\theta_{2}$ increases, $\operatorname{sinc}\left(\left(v_{x}-v_{x}^{\prime}\right) L\right)$ quickly tends to zero and so does $\mathrm{E}\left(\rho\left(\rho^{\prime}\right)^{*}\right)$.

In Figure 3.5, the incident angle $\theta_{1}=5.625^{\circ}$, therefore, the incident wave is nearly vertical to the surface. Compared to the large shift of the maximum power off the specular direction 


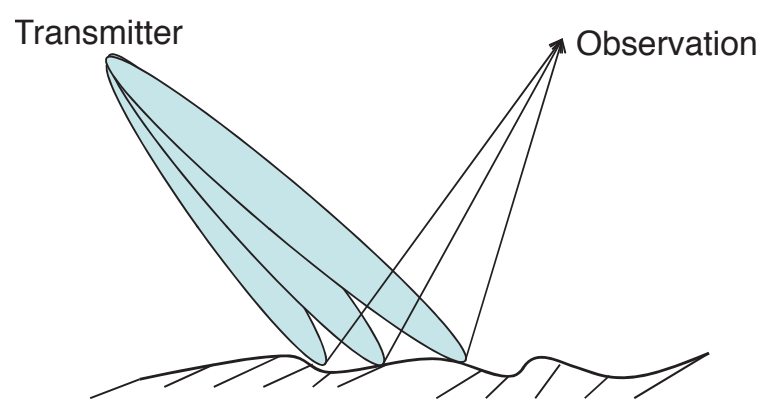

Figure 3.7: Illustration of beam oscillation technique.

in Figure 3.4 (c), it is observed in Figure 3.5 (c) that the maximum reflected power is achieved when the reflected ray is near to the specular direction. Moreover, the scattering power distribution in (c) looks similar to that in (a) except having greater width. The figure shows that when the incident angle is small, the scattering power is approximately symmetric around the y axis.

In Figure 3.6, The incident angle $\theta_{1}=45^{\circ}$, and the reflected angle $\theta_{2}=22.5^{\circ}$. It is seen that $\mathrm{E}\left(\rho\left(\rho^{\prime}\right)^{*}\right)$ 's peak value for $\sigma=10 \lambda$ in (b) is larger than that for $\sigma=0.5 \lambda$ in (d), while the opposite result occurs in Figure 3.4 and Figure 3.5. This can be explained by the fact that the scattered energy of slightly rough surface highly concentrates around the specular direction; thus, the energy scattered in other directions is minimal. Meanwhile, the scattered energy of very rough surface distributes in various directions. Therefore, lower energy in off-specular direction of slightly rough surface leads to lower correlation.

Here, we observed that this channel model involves mainly three parameters to describe the scatterers: the length $L$ of the scattering plane, the correlation distance $T$ and the standard deviation $\sigma$ of the rough surface height. Now, we explain how these parameters are 
related to a real channel scenario. In addition, we propose techniques for estimating those parameters.

The rough surface parameters are a function of the wavelength and the structure of surface roughness. The rough surfaces encountered in the nature, e.g., terrain, sea, atmospheric layers, can be best described by the statistical distribution of their deviation from the mean level. For radio communication, hilly areas or moving passengers along crowded downtown streets can be modeled as random rough surfaces as well. The roughness ÏČ represents the standard deviation of the height of the surface compared to the wavelength. The correlation distance $\mathrm{T}$ indicates how close the hills and valleys of the surface are crowded together. The length, $\mathrm{L}$, of the scattering plane is determined by the size of the first Frensnel zone ([58, Ch.2.2]).

Different techniques can be proposed to estimate the scatterer parameters. Some include: frequency diversity, beam oscillation and scattered power distribution measurement. We now briefly describe how they work. Frequency diversity method is based on the fact that the scatterer roughness behavior varies with frequency (wavelength). For example, if the roughness is in the order of one meter and the wavelength is in the order of 100 meters, the surface exhibits specular behaviors; thus, reflects the incident power almost in one direction. However, the same scatterer would act as a rough surface when the wavelength is in the order of one meter. In other words, the scattered power varies as the wavelength of the transmitted signal changes due to the fact that the irregularity of a surface depends on 
the wavelength of the impinging signal. Thus, we may illuminate the surface of scatterer with a narrow beam signal modulated using different frequency ranges and measure the received power at the receiver. According to the above explanation, it is expected that the received power varies with frequency differently for different scatterer roughness $\sigma$. Thus, the generated curve can be compared with a family of pre-measured curves with different values of the desired parameters.

Beam oscillation method basically uses antenna array to steer beams toward a series of incident directions upon the surface as shown in Figure 3.7. A probe is placed at some points to measure the scattered power so as to get a curve versus different incident and scattered angles. Then Equations (3.28) and (3.33) can be applied to solve the desired parameters. Scattered power distribution measurement is similar to the beam oscillation except that the incident beam is fixed and the probe moves around to measure the scattered power along different directions. The full investigation of those methods is out of the scope of this chapter. Interested readers are referred to [63] where the relations between surface statistics and the scattered wave statistics are surveyed.

Note that the proposed model contains the rich scattering scenario. In a richly scattered environment, the channel matrix elements have identical independent Gaussian distribution. The physical basis of this model is that there are a significant number of equal-energy multipaths in each of the resolvable angular bins [51], which roughly corresponds to a large spread out of the scattered signal from random rough surfaces. Because large roughness 


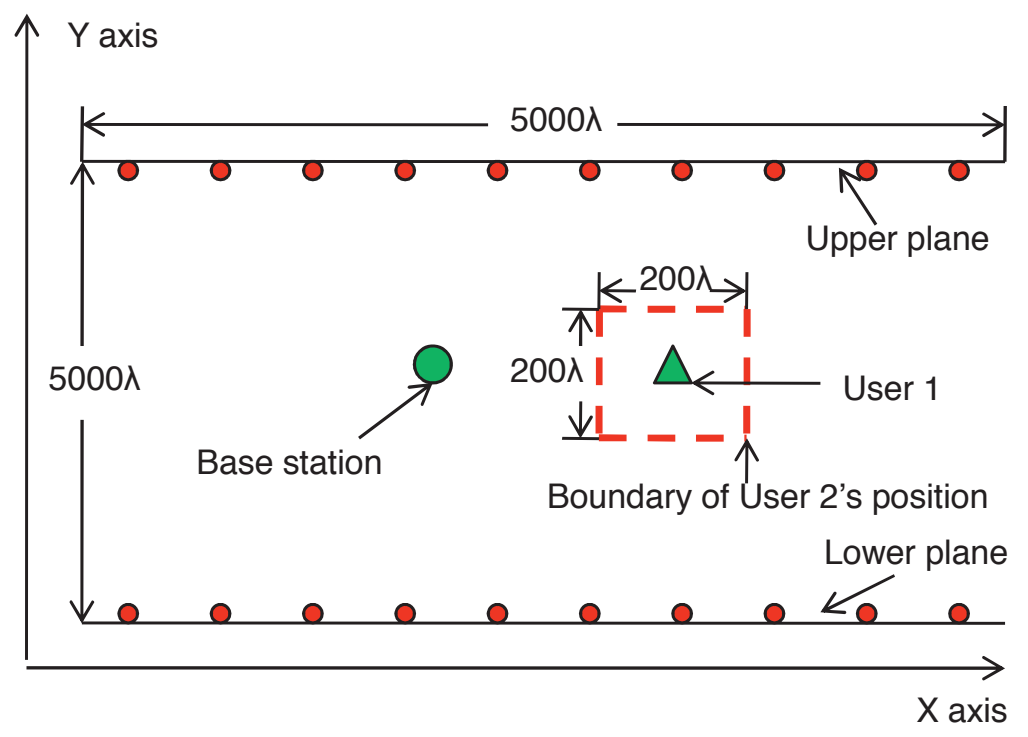

Figure 3.8: Simulation set-up.

$\sigma$ leads to the large spread out as shown in Figure 3.4, richly scattered environment is achieved when the roughness $\sigma$ is large.

\subsection{Numerical results and analysis}

In this section, we present simulation results of the channel coefficient correlation of interand intra-user antenna pairs for both LOS and NLOS situations, i.e., $\gamma_{m n, s t}^{(1)(2) \mathrm{NLOS}}, \gamma_{m n, s t}^{(1)(2) \mathrm{LOS}}$, $\gamma_{m n, s t}^{(1)(1) \operatorname{NLOS}}, \gamma_{m n, s t}^{(1)(1) \operatorname{LOS}}$ in (3.9)-(3.12).

A schematic scattering environment is shown in Figure 3.8. The primary scattering environment consists of two planes which are 5000ג apart and the active scattering region of both planes is $5000 \lambda$ in length. The active scattering region is segmented equally and the center of each part is denoted by the small square on the upper and lower planes. The base 
station (BS) is placed at the point $(0,0)$ and User 1 is at $(1000 \lambda, 0)$. The position of User 2 varies in a square region of dimension $200 \lambda \times 200 \lambda$, with the boundary denoted by the red bold dashed line. User 1 is at the center of the square.

The parameters for the following simulation results are listed here or specified otherwise: $\lambda=10 \mathrm{~cm}, L=250 \lambda, T=25 \lambda, d_{r}=d_{t}=0.5 \lambda$, and in (3.7), $K_{1}=K_{2}=K=1$. Here, $K$ essentially represents the power ratio of LOS and NLOS. Simulations are performed for different roughness, and different distances between User 1 and BS.

The two dimensional spatial cross-user correlation $\gamma_{11,11}^{(1)(2) N L O S}$ and $\gamma_{11,11}^{(1)(2) L O S}$ for NLOS and LOS, respectively, is depicted in Figure 3.9 when $\sigma=0.5 \lambda, 10 \lambda, \mathrm{D}(\mathrm{BS}$, User 1$)=3000 \lambda$ which is the distance between BS and User 1. Compared to the correlation corresponding to $\sigma=0.5 \lambda$, the correlation for higher roughness $\sigma=10 \lambda$ spreads out to neighboring parts and has lower values (darker squares). This is consistent with the scattered energy distributions in Figure 3.4 where surfaces with higher roughness spread out the signal into various directions and each direction receives less power.

In order to see the impact of the topography, we consider another set-up: The distance of BS and User 1 is set to $10000 \lambda$. Simulation and visualization are repeated and the results can be found in Figure 3.10. It is seen that a shining cross appears in b) NLOS correlation. This happens when the two users are in line with a significant scattering path and agrees with the conclusion illustrated in Figure 3.4: the correlation tends to zero very fast when the separation of the scattering angles increases. Therefore, topography plays a main role 


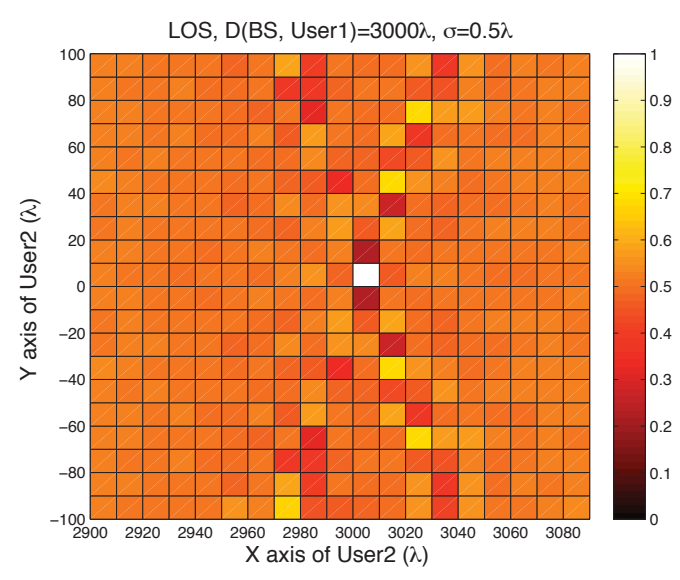

(a) $\sigma=0.5 \lambda$, LOS

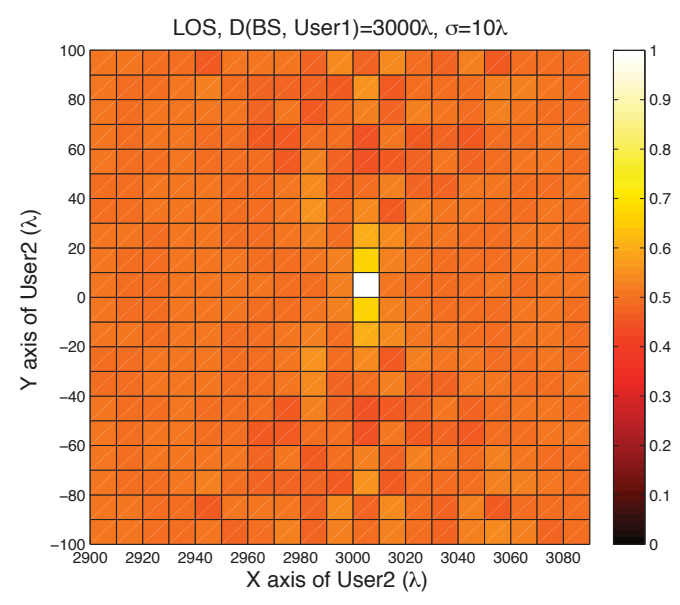

(c) $\sigma=10 \lambda$, LOS

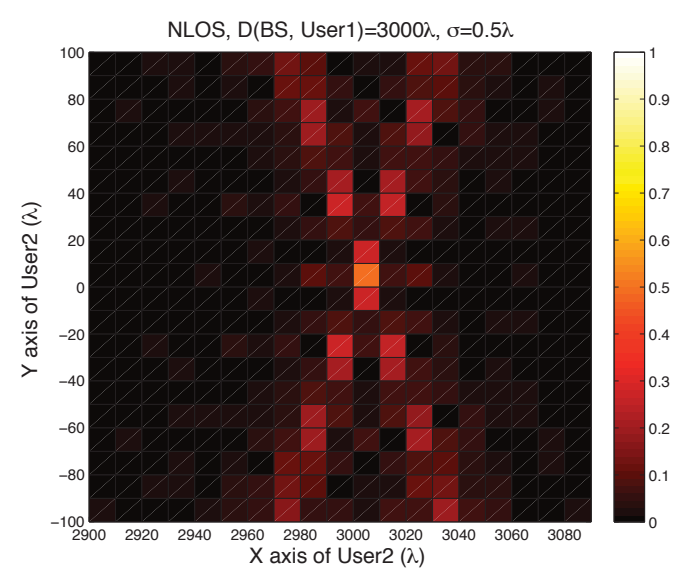

(b) $\sigma=0.5 \lambda$, NLOS

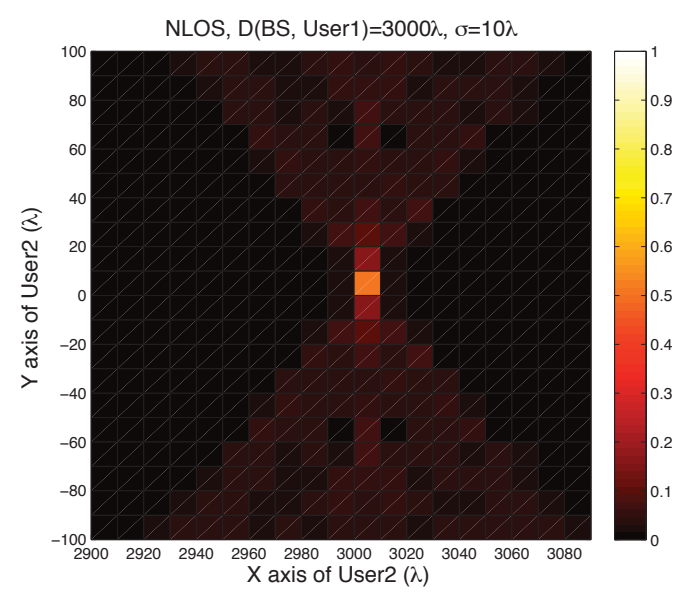

(d) $\sigma=10 \lambda$, NLOS

Figure 3.9: Comparison of LOS and NLOS spatial cross-user correlation for $\sigma=$ $0.5 \lambda, 10 \lambda$ when $\mathrm{D}(\mathrm{BS}$, User 1$)=3000 \lambda$.

in the look of the spatial correlation. Both in Figure 3.9 and Figure 3.10, LOS correlation is generally larger than the NLOS and has less sharp variations. This reveals the stabilization effect of LOS signal.

Figure 3.11 shows the comparison of correlation for NLOS and LOS when User 2's position is along $\mathrm{X}$ axis ( $\mathrm{Y}$ coordinate is 0 ). It is observed that in most area, LOS correlation is much larger than the NLOS correlation except for the case that two users are closely located. It is 


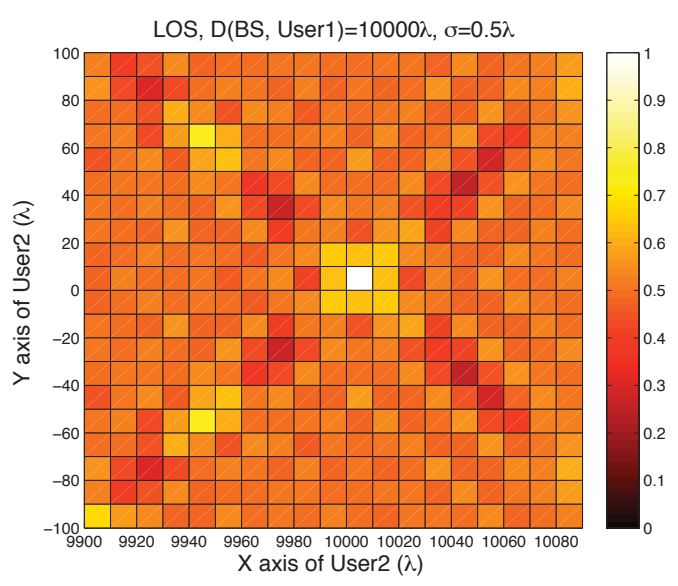

(a) $\sigma=0.5 \lambda, \operatorname{LOS}$

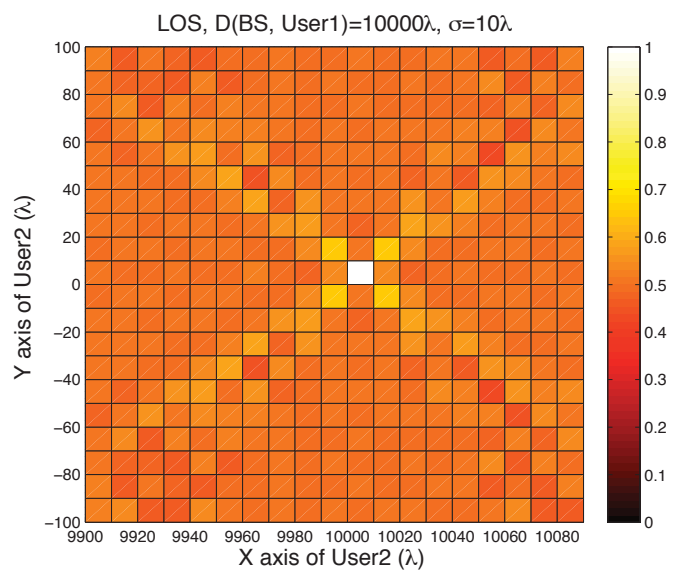

(c) $\sigma=10 \lambda, \operatorname{LOS}$

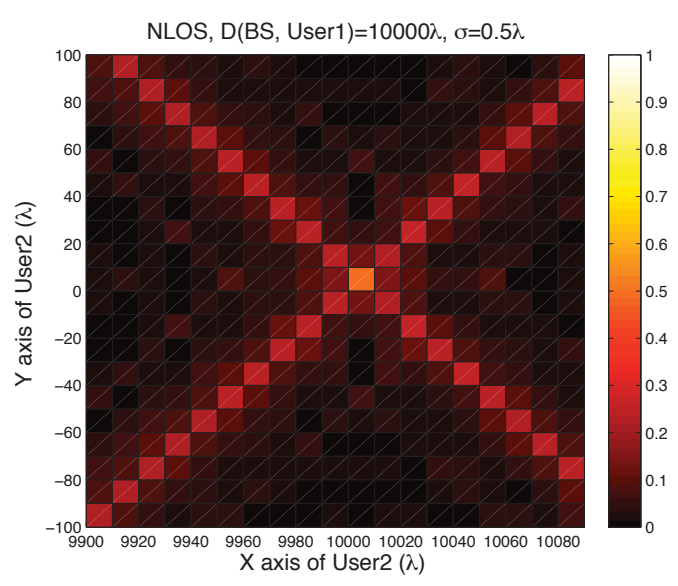

(b) $\sigma=0.5 \lambda$, NLOS

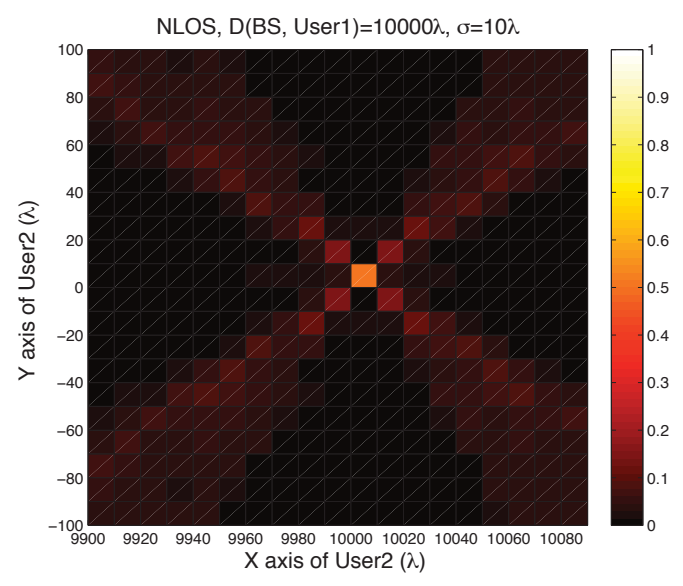

(d) $\sigma=10 \lambda$, NLOS

Figure 3.10: Comparison of LOS and NLOS spatial cross-user correlation for $\sigma=$ $0.5 \lambda, 10 \lambda$ when $\mathrm{D}(\mathrm{BS}$, User 1$)=10000 \lambda$.

also observed that NLOS correlation has more fluctuations than the LOS correlation. This is basically resulted from the absence of the deterministic LOS component.

Spatial intra-user correlation $\gamma_{11,12}^{(1)(1) \text { NLOS }}$ and $\gamma_{11,12}^{(1)(1) \text { LOS }}$ versus BS's antenna spacing $d_{t}$ is shown in Figure 3.12 for $K=1$ and $K=4$. The envelopes of LOS are generally above those of NLOS. The correlation is lower for very rough surface $(\sigma=10 \lambda)$, which may be probably because that lower power is received for large roughness. When $K$ factor is 


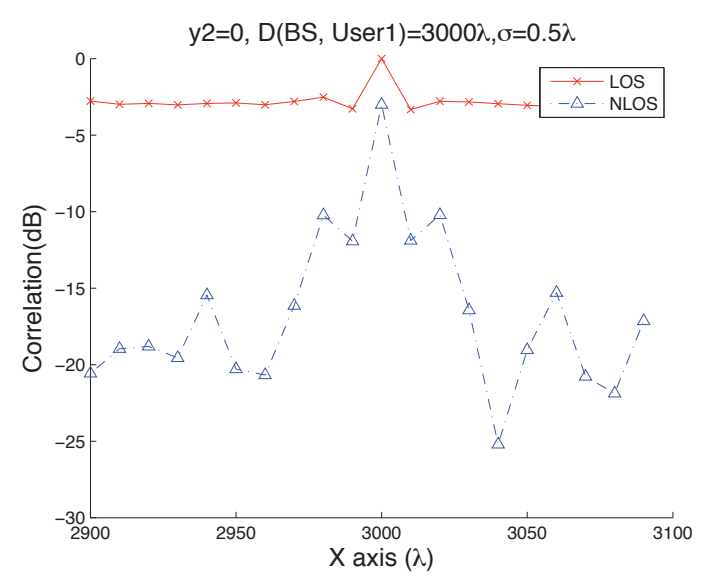

(a) $\sigma=0.5 \lambda, \mathrm{D}(\mathrm{BS}$, User 1$)=3000 \lambda$ y2 $=0, D(B S$, User 1$)=10000 \lambda, \sigma=0.5 \lambda$

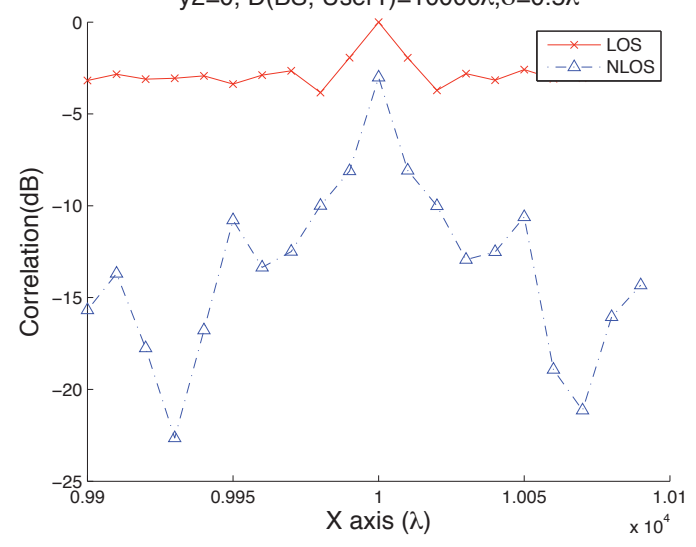

(c) $\sigma=0.5 \lambda, \mathrm{D}(\mathrm{BS}$, User 1$)=10000 \lambda$

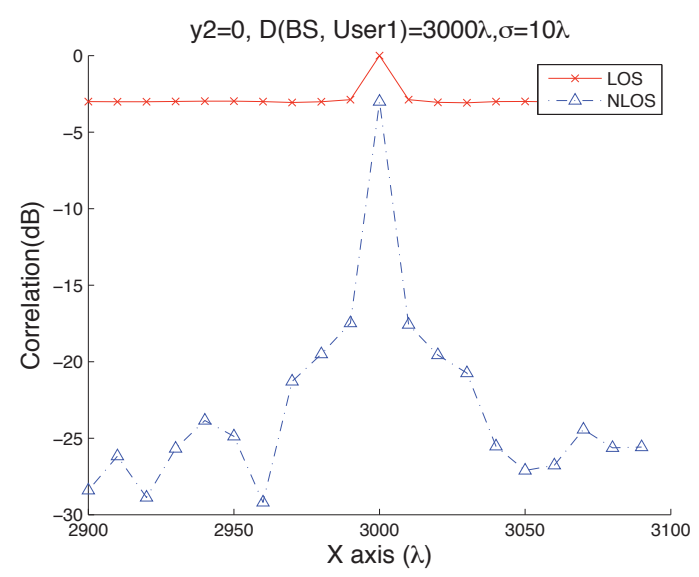

(b) $\sigma=10 \lambda, \mathrm{D}(\mathrm{BS}, \mathrm{User} 1)=3000 \lambda$ $\mathrm{y} 2=0, \mathrm{D}(\mathrm{BS}$, User 1$)=10000 \lambda, \sigma=10 \lambda$

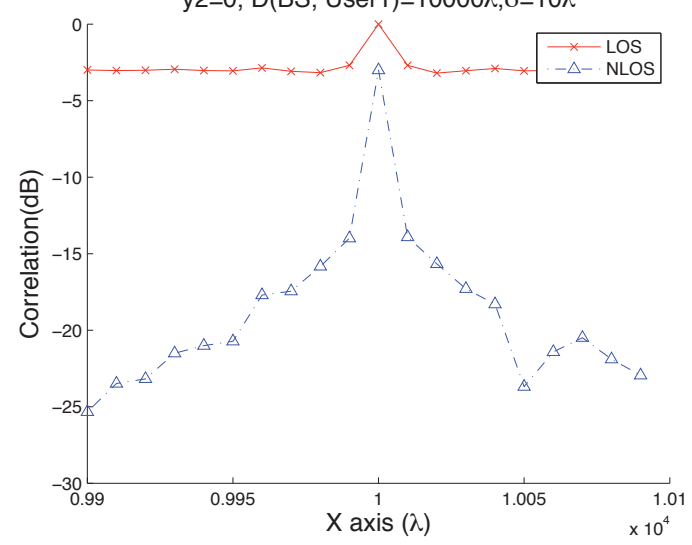

(d) $\sigma=10 \lambda, \mathrm{D}(\mathrm{BS}$, User 1$)=10000 \lambda$

Figure 3.11: Comparison of LOS and NLOS spatial cross-user correlation along $\mathrm{X}$ axis $(\mathrm{Y}$ coordinate $=0$ ) for $\sigma=0.5 \lambda, 10 \lambda$ when $\mathrm{D}(\mathrm{BS}, \mathrm{User} 1)=3000 \lambda, 10000 \lambda$.

increased to 4, there is a big separation between LOS and NLOS as depicted in (c) and (d).

The fluctuation range of LOS in (c) and (d) is smaller than that in (a) and (b), which again shows the stabilization effect of LOS signals.

If User 1's antenna spacing varies, the behavior of intra-user correlation $\gamma_{11,21}^{(1)(1) \mathrm{NLOS}}$ and $\gamma_{11,21}^{(1)(1) \operatorname{LOS}}$ is identical to that in Figure 3.12, because BS and User 1 are located symmetrically about the scattering plane as shown in Figure 3.8 . 

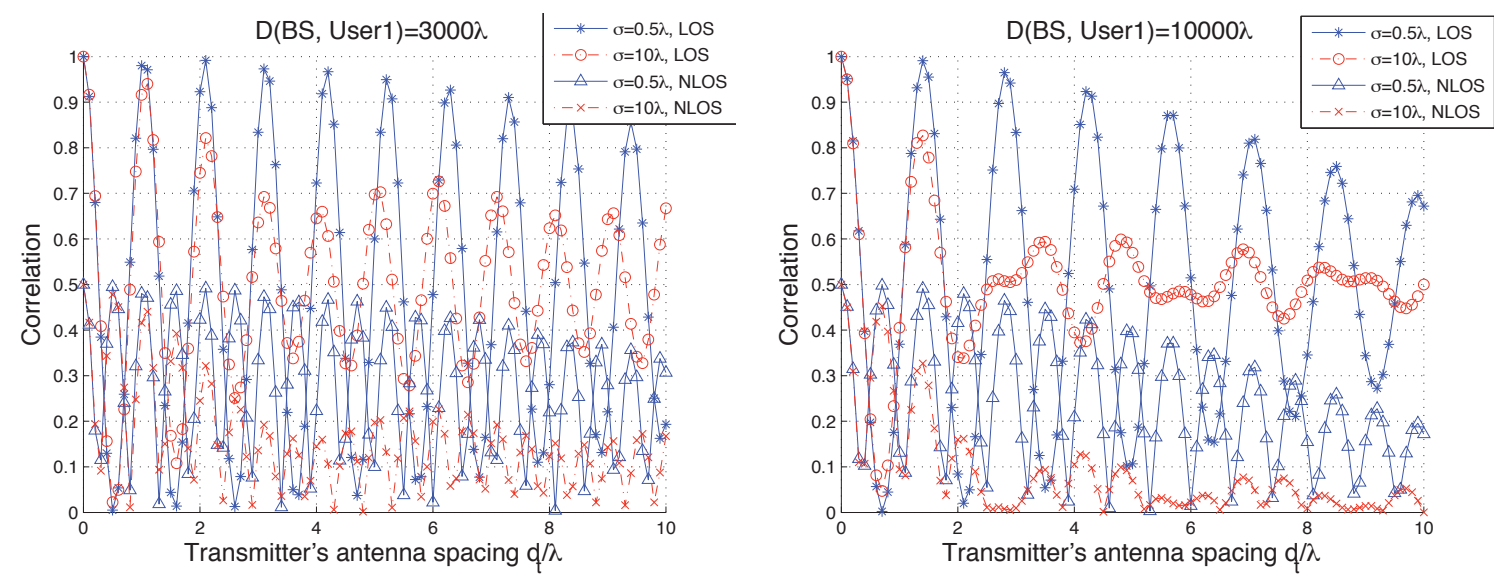

(a) $\mathrm{D}(\mathrm{BS}$, User 1$)=3000 \lambda, K=1$ $\mathrm{D}(\mathrm{BS}$, User1 $)=3000 \lambda$

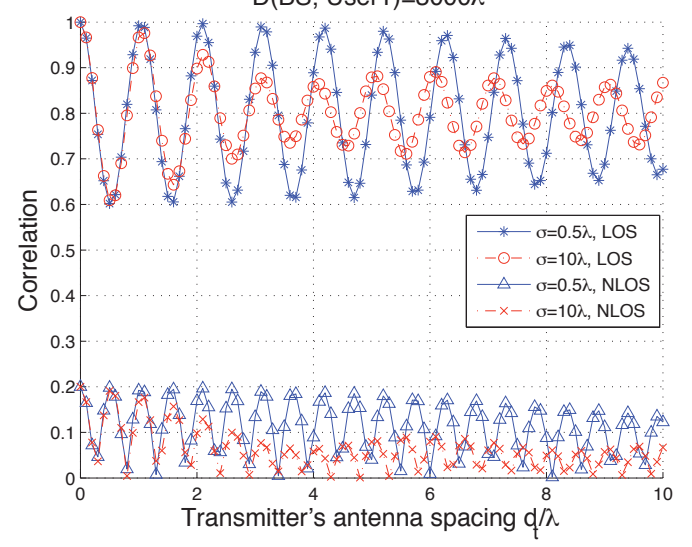

(b) $\mathrm{D}(\mathrm{BS}, \mathrm{User} 1)=10000 \lambda, K=1$

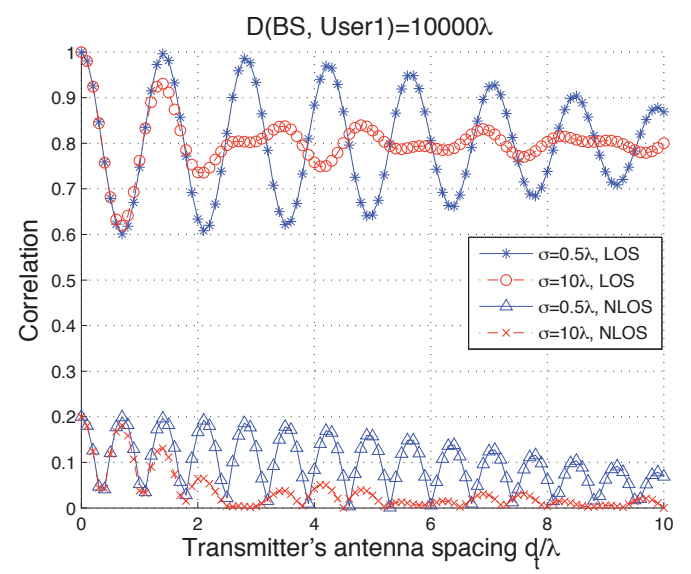

(c) $\mathrm{D}(\mathrm{BS}$, User 1$)=3000 \lambda, K=4$

(d) $\mathrm{D}(\mathrm{BS}$, User 1$)=10000 \lambda, K=4$

Figure 3.12: Comparison of LOS and NLOS spatial intra-user correlation versus BS's antenna spacing for $\sigma=0.5 \lambda, 10 \lambda$ when $K=1,4$.

In the above intra-user correlation analysis, the antenna arrays at both the receiver and the transmitter are perpendicular to the rough surfaces as shown in Figure 3.2. If the antenna orientation of User 1 (receiver) is changed, intra-user correlation may also change. Here, we study the impact of the antenna orientation of User 1 by considering a case: the BS's antenna array remains perpendicular to the surfaces while User 1's antenna array is mounted in parallel with the surfaces. 


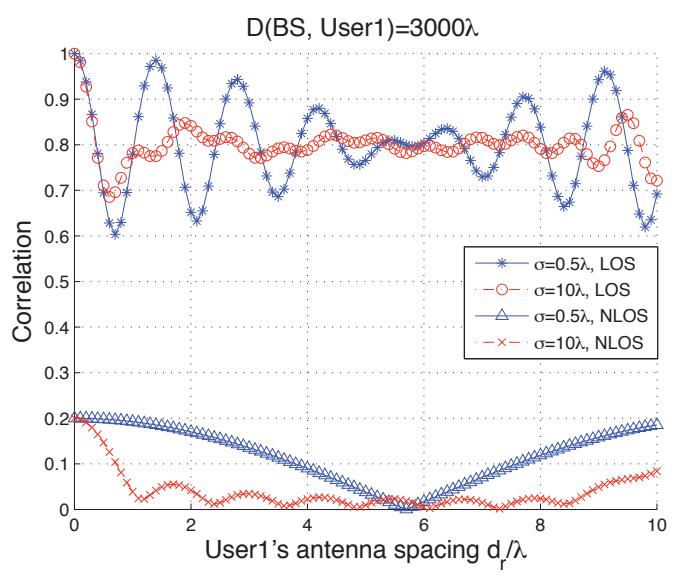

(a) $\mathrm{D}(\mathrm{BS}$, User 1$)=3000 \lambda, K=4$

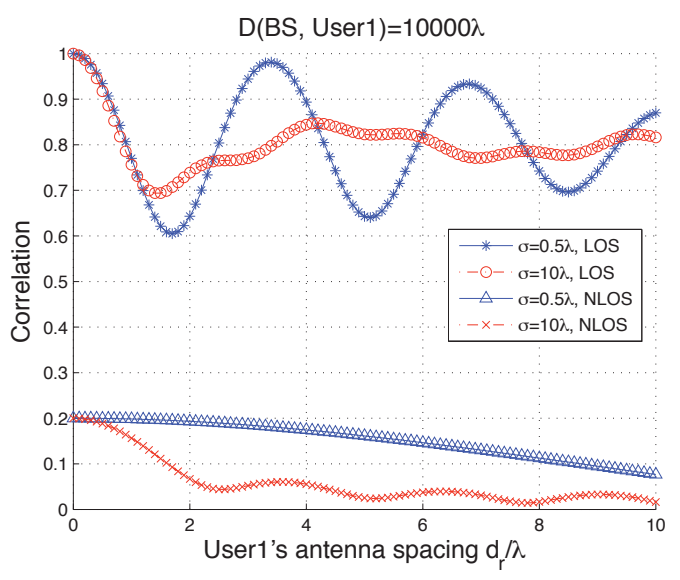

(b) $\mathrm{D}(\mathrm{BS}$, User 1$)=10000 \lambda, K=4$

Figure 3.13: LOS and NLOS spatial intra-user correlation for $\sigma=0.5 \lambda, 10 \lambda$ when User 1's antenna array is parallel to the surfaces.

The intra-user correlation for the parallel antenna array is shown in Figure 3.13. In this figure, the correlation curves of NLOS have no or sparse fluctuations, but the fluctuations are found in the LOS curves (more obvious for $\sigma=0.5 \lambda$ ). This is because the LOS component produces phase shifts along the direction of antenna array as a result of the alignment between LOS direction and User 1's antenna array. Looking back to Figure 3.12 (c) and (d), both LOS and NLOS correlation curves have similar fluctuations. This is because, due to the perpendicularity between the LOS direction and the antenna array, the LOS component does not produce any phase shift along the direction of antenna array.

\subsection{Conclusion}

In this chapter, we proposed a novel approach to investigate the spatial correlation across antenna elements within one user and across users in multiuser MIMO wireless communication systems. The intra-user and inter-user correlations are theoretically derived and 
numerically evaluated.

Simulation results represent that higher roughness leads to lower correlation. In addition, topography, i.e., whether two users are in line with the significant scattering path, plays a main role in the shape of the cross-user correlation.

For intra-user and inter-user cases, it is observed that LOS signals drastically boosts the correlation and has a more flat trajectory in space. This property can be incorporated to discriminate LOS signals from NLOS. 


\section{Chapter 4}

\section{NLOS Identification via Phase Difference Statis-}

\section{tics across Two Antenna Elements}

This chapter proposes and investigates the performance of a new NLOS identification technique for multiple antenna systems that is based on the phase difference across two antenna elements. A phase wrapping selection algorithm is proposed to calculate the phase difference variance across two antenna elements. A theoretical relationship is maintained between the phase difference variance and the Rician $K$-factor. The proposed $K$ estimator requires an uncorrelated phase across antenna elements. The validity of this assumption is verified via channel modeling simulations. Then, a hypothesis test on the $K$-factor is formed to identify NLOS situations. The prior distributions of $K$-factor under LOS and NLOS conditions, and the $K$-factor threshold which are used to distinguish LOS and NLOS situation are derived. The impact of shadowing on the performance of the proposed NLOS 
identification method is studied. The performance of the proposed phase difference based $K$ estimator is compared with that of the envelope-based $K$ estimator.

\subsection{Introduction}

This chapter introduces a novel NLOS identification technique that is based on the statistics of the phase difference of two signals received across two antenna elements in an antenna array. The received signal is usually modeled as a summation of a LOS component and a diffusive component. When the LOS path is blocked, i.e., NLOS condition, $K$-factor that is the ratio of LOS power to the diffusive one, is usually very small compared to that of LOS condition [30]. This is due to the fact that the LOS signal usually suffers a large attenuation due to the blockage between the transmitter and receiver.

In this chapter, the phase difference variance $\sigma_{\Delta \phi}^{2}$ is analytically derived, assuming $u n$ correlated phase across antenna elements. It is depicted that $\sigma_{\Delta \phi}^{2}$ is a function of Rician $K$-factor. When $K=0, \sigma_{\Delta \phi}^{2}$ has the maximum value of $2 \pi^{2} / 3$, and $\sigma_{\Delta \phi}^{2}$ decreased as $K$ increases. Therefore, the variance $\sigma_{\Delta \phi}^{2}$ can be used to form a binary hypothesis test for NLOS identification based on Rician $K$-factor. Here, distributions of $K$ for LOS and NLOS are computed, and to identify LOS from NLOS, a threshold for $K$ is computed too. The hypothesis test requires a limited number of phase difference samples. Thus, it functions fast and its complexity is low. 
In addition, the chapter proposes a new Rician $K$-factor estimator based on $\sigma_{\Delta \phi}^{2}$. The performance of the phase difference based $K$ estimator is compared to a benchmark envelope based $K$ estimator.

Moreover, the assumption of uncorrelated phase across antenna elements is investigated for both circular and elliptic scattering channels.

The remainder of the chapter is organized as follows: Section 4.2 presents the received signal model and the Rician $K$-factor estimator based on the phase difference variance $\sigma_{\Delta \phi}^{2}$. Section 4.3 formulates the problem of NLOS identification based on Rician $K$-factor. Section 4.4 presents numerical justification on the uncorrelated phase assumption. Section 4.5 provides numerical evaluation of the performance of $K$-factor estimator based on the phase difference and the performance of NLOS identification based on $K$-factor. Some concluding remarks are made in Section 4.6.

\subsection{Received signal model and $K$-factor estimator}

A co-installed synchronized two-antenna system shown in Figure 4.1 can be used for the proposed identification method. The far region scenario is assumed and therefore the impinging wave on the antenna array is plane wave. In this system, two antennas are installed with a fixed spacing and they share the same local oscillator. Therefore, the phase differ- 


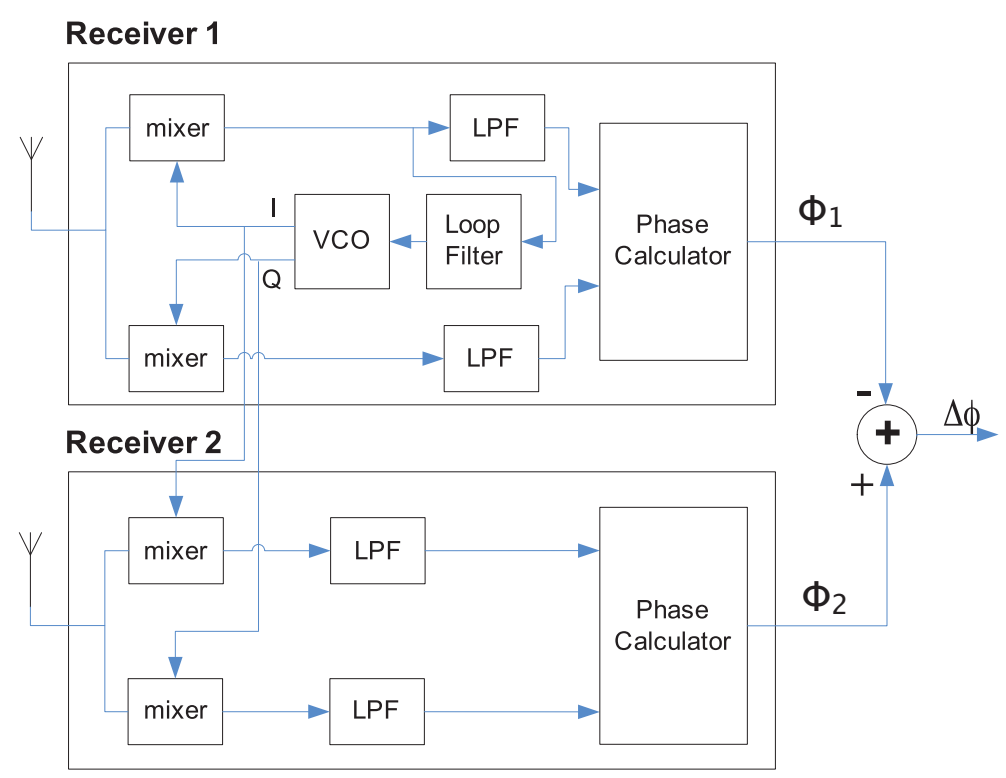

Figure 4.1: Two-antenna receiver.

ence is

$$
\Delta \phi=\phi_{2}-\phi_{1}, \quad \phi_{1}, \phi_{2} \in[-\pi, \pi]
$$

where $\phi_{1}$ and $\phi_{2}$ are the phases of $r_{1}$ and $r_{2}$, the received signals of the two antennas. As shown in Figure 4.2, $r_{1}=r_{\mathrm{LOS} 1}+r_{\mathrm{DIF} 1}$ and $r_{2}=r_{\mathrm{LOS} 2}+r_{\mathrm{DIF} 2}$ where the subscript LOS and DIF denotes the LOS and diffusive components. Now, using Figure 4.2 and considering the range of $\phi_{1}$ and $\phi_{2}$ in (4.1), the phase difference $\Delta \phi$ corresponds to

$$
\begin{aligned}
\Delta \phi & =I_{[-\pi, \pi]}\left(\phi_{\mathrm{LOS} 1}+2 \pi d \cos \theta / \lambda+\Delta \phi_{2}\right)-I_{[-\pi, \pi]}\left(\phi_{\mathrm{LOS} 1}+\Delta \phi_{1}\right) \\
& =\Delta \phi_{2}-\Delta \phi_{1}+2 \pi d \cos \theta / \lambda+X
\end{aligned}
$$




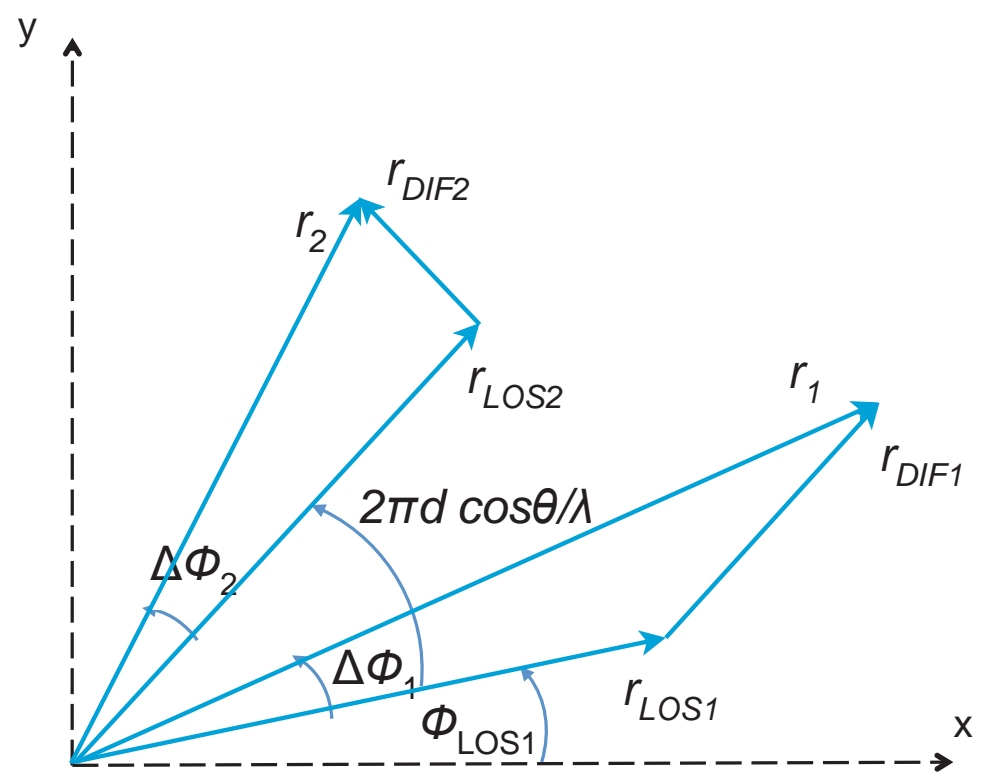

Figure 4.2: Received signals in vector space.

where $I_{[-\pi, \pi]}(\cdot)$ wraps the phase to the range $[-\pi, \pi], \phi_{\mathrm{LOS} 1}$ is the phase of $r_{\mathrm{LOS} 1}, \Delta \phi_{2}$ is the angle shift from $r_{\mathrm{LOS} 2}$ caused by $r_{\mathrm{DIF} 2}, \Delta \phi_{1}$ is similarly defined, $d$ is the antenna spacing, $\theta$ is the DOA, i.e., the angle made by the direction of the arriving signal and the antenna array, and $\lambda$ is the wavelength. $X$ is a discrete random variable which takes values from $\{\cdots-4 \pi,-2 \pi, 0,2 \pi \cdots\}$ and ensures that the range of $\Delta \phi$ in (4.1) would stay within $[-2 \pi, 2 \pi]$. Note that the range of $\phi_{1}$ and $\phi_{2}$ in Figure 4.1 should stay within $[-\pi, \pi]$. Thus, the range of $\Delta \phi$ would be $[-2 \pi, 2 \pi]$.

It is assumed that the DOA of LOS signal $(\theta)$ does not change within the sampling duration. Hence, $\phi_{\mathrm{LOS} 1}$ and $2 \pi d \cos \theta / \lambda$ in (4.2) are fixed but unknown and could accept any value within $-\pi$ to $\pi$ range. With certain probability, $\phi_{\mathrm{LOS} 1}$ is around $(2 n+1) \pi$ where $n$ is an integer number, and the wrapping function may add multiples of $\pm 2 \pi$ to $\phi_{\mathrm{LOS} 1}+\Delta \phi_{1}$, giving rise to the randomness of $X$. In order to make the variance of $\Delta \phi$ is equal to the 


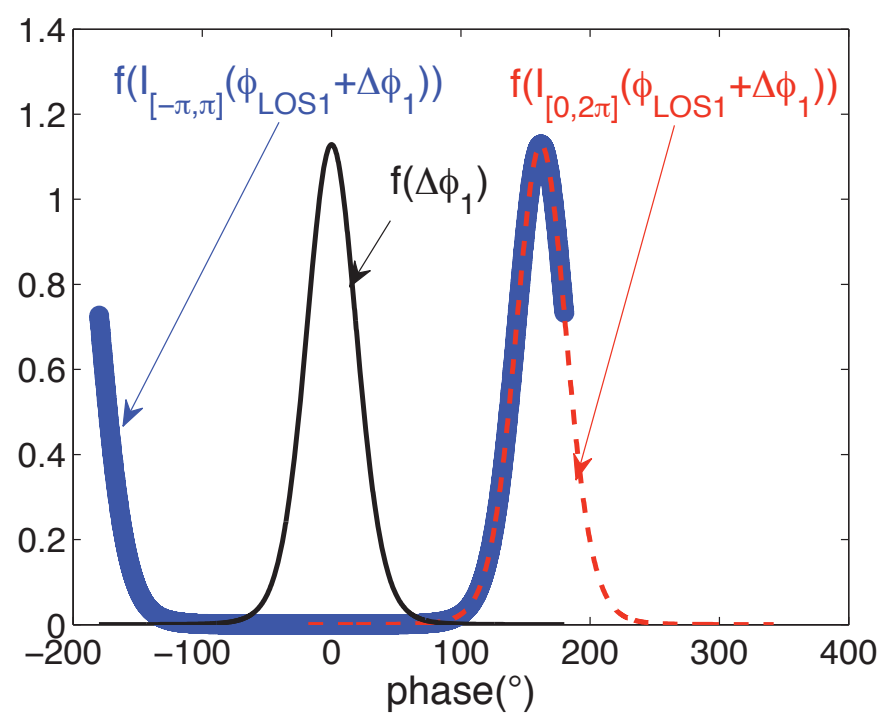

Figure 4.3: PDFs of $\Delta \phi_{1}, I_{[-\pi, \pi]}\left(\phi_{\mathrm{LOS} 1}+\Delta \phi_{1}\right)$ and $I_{[0,2 \pi]}\left(\phi_{\mathrm{LOS} 1}+\Delta \phi_{1}\right)$ under the case $\phi_{\mathrm{LOS} 1} \approx \pi$

variance of $\Delta \phi_{2}-\Delta \phi_{1}$, the randomness of $X$ has to be eliminated.

When $\phi_{\mathrm{LOS} 1} \approx \pi$, the probability density functions (PDFs) of $\Delta \phi_{1}, I_{[-\pi, \pi]}\left(\phi_{\mathrm{LOS} 1}+\Delta \phi_{1}\right)$ and $I_{[0,2 \pi]}\left(\phi_{\mathrm{LOS} 1}+\Delta \phi_{1}\right)$ are shown in Figure 4.3. Using $[-\pi, \pi]$ wrapping, the PDF of $I_{[-\pi, \pi]}\left(\phi_{\mathrm{LOS} 1}+\Delta \phi_{1}\right)$ represented by the bold line in Figure 4.3 has a significant component around $-\pi\left(-180^{\circ}\right)$ due to wrapping. Thus, it fails to preserve the shape of $f\left(\Delta \phi_{1}\right)$ indicated by the thin solid line in Figure 4.3. This results that the variance of $I_{[-\pi, \pi]}\left(\phi_{\operatorname{LOS} 1}+\Delta \phi_{1}\right)$ (which is 4.9202) is larger than that of $\Delta \phi_{1}$ (which is 0.1528). Instead, using $[0,2 \pi]$ wrapping, the distribution of $I_{[0,2 \pi]}\left(\phi_{\mathrm{LOS} 1}+\Delta \phi_{1}\right)$ represented by the dashed line in Figure 4.3 has a similar shape of the distribution of $\Delta \phi_{1}$. As a result, $I_{[0,2 \pi]}\left(\phi_{\mathrm{LOS} 1}+\Delta \phi_{1}\right)$ has a variance of 0.1528 which is close to that of $\Delta \phi_{1}$.

The above analysis shows that improper wrapping enlarges the variance, while proper 
wrapping preserves the variance. Inversely, proper wrapping should be identified as having the smallest variance. The algorithm of selecting proper wrapping to calculate $\Delta \phi$ in (4.2) is summarized in the following:

1. Compute the variance of $I_{[-\pi, \pi]}\left(\phi_{\mathrm{LOS} 1}+2 \pi d \cos \theta / \lambda+\Delta \phi_{2}\right)$ and the variance of $I_{[0,2 \pi]}\left(\phi_{\mathrm{LOS} 1}+2 \pi d \cos \theta / \lambda+\Delta \phi_{2}\right)$, then choose the wrapping resulting in smaller variance.

2. Compute the variance of $I_{[-\pi, \pi]}\left(\phi_{\mathrm{LOS} 1}+\Delta \phi_{1}\right)$ and the variance of $I_{[0,2 \pi]}\left(\phi_{\mathrm{LOS} 1}+\right.$ $\left.\Delta \phi_{1}\right)$, then choose the wrapping resulting in smaller variance.

Applying this algorithm, the variance of $(2 \pi d \cos \theta / \lambda+X)$ is maintained much smaller than $\sigma_{\Delta \phi_{i}}^{2}, i \in[1,2]$. This point will be verified in Section 4.5 where we present the performance of this technique. Accordingly, the variance of $\Delta \phi$ would be well approximated by the variance of $\Delta \phi_{2}-\Delta \phi_{1}$, and would correspond to

$$
\sigma_{\Delta \phi}^{2}=\sigma_{\Delta \phi_{1}}^{2}+\sigma_{\Delta \phi_{2}}^{2}-2 \operatorname{cov}\left(\Delta \phi_{1} \Delta \phi_{2}\right)
$$

where $\operatorname{cov}(\cdot)$ denotes the covariance operation. Using Figure $4.2, \Delta \phi_{1}=\operatorname{angle}\left(r_{1} / r_{\text {LOS1 } 1}\right)=$ $\operatorname{angle}\left(1+r_{\mathrm{DIF} 1} / r_{\mathrm{LOS} 1}\right), \Delta \phi_{2}=\operatorname{angle}\left(1+r_{\mathrm{DIF} 2} / r_{\mathrm{LOS} 2}\right) \cdot r_{\mathrm{DIF} 1}$ and $r_{\mathrm{DIF} 2}$ is assumed to be independent, which is a reasonable assumption for rich scattering environments, and large antenna spacing (greater than half wavelength) [64]. Therefore, $\Delta \phi_{1}$ and $\Delta \phi_{2}$ can also be considered independent, i.e., $\operatorname{cov}\left(\Delta \phi_{1} \Delta \phi_{2}\right)=0$. The uncorrelated phase assumption 
for circular and elliptic scattering environment will be further studied via simulations in Section 4.4.

Assuming $r_{\mathrm{DIF} 2}$ is complex Gaussian distributed, the PDF of $\Delta \phi_{1}$ is derived as (the detail derivation is in Appendix C)

$f\left(\Delta \phi_{1}\right)=\frac{\exp (-K)}{2 \pi}+\sqrt{\frac{K}{\pi}} \cos \Delta \phi_{1} \exp \left(-K \sin ^{2} \Delta \phi_{1}\right) \times Q\left(-\sqrt{2 K} \cos \Delta \phi_{1}\right), \quad \Delta \phi_{1} \in[-\pi, \pi]$

where $K$ is the Rician factor, i.e., $K=\left|r_{\mathrm{LOS} 1}\right|^{2} / E\left[\left|r_{\mathrm{DIF} 1}\right|^{2}\right]$ and $Q(\cdot)$ is the complementary error function defined as $Q(x)=\int_{x}^{\infty} e^{-u^{2} / 2} / \sqrt{2 \pi} d u$. Assuming $r_{2}$ has the same $K$ as $r_{1}$, the distribution of $\Delta \phi_{2}$ would be identical to that of $\Delta \phi_{1}$. Thus, $\sigma_{\Delta \phi_{1}}^{2}=\sigma_{\Delta \phi_{2}}^{2}$ and using (4.2), $\sigma_{\Delta \phi}^{2}$ corresponds to

$$
\sigma_{\Delta \phi}^{2}=2 \sigma_{\Delta \phi_{1}}^{2}=2 \int_{-\pi}^{\pi} \Delta \phi_{1}^{2} f\left(\Delta \phi_{1}\right) d \Delta \phi
$$

Because a closed form expression of (4.5) does not exist, $\sigma_{\Delta \phi}^{2}$ is numerically computed and plotted in Figure 4.4. The estimation of $K$ can be obtained using a lookup table or be approximated by a closed form expression. Figure 4.4 plots $g(K) \triangleq 1 / \sigma_{\Delta \phi}^{2}$. Here, $g(K)$ is well approximated by piece-wise low-order polynomial functions of $K, g_{1}(K)=$ $a K^{2}+b K+c$ when $K \in[0,5]$ and $g_{2}(K)=\beta K+\gamma$ when $K \in[5,30]$. The coefficients $a, b$, $c, \beta$ and $\gamma$ are computed by fitting $g_{1}(K)$ and $g_{2}(K)$ to $g(K)$ in a least-square sense. Then, 

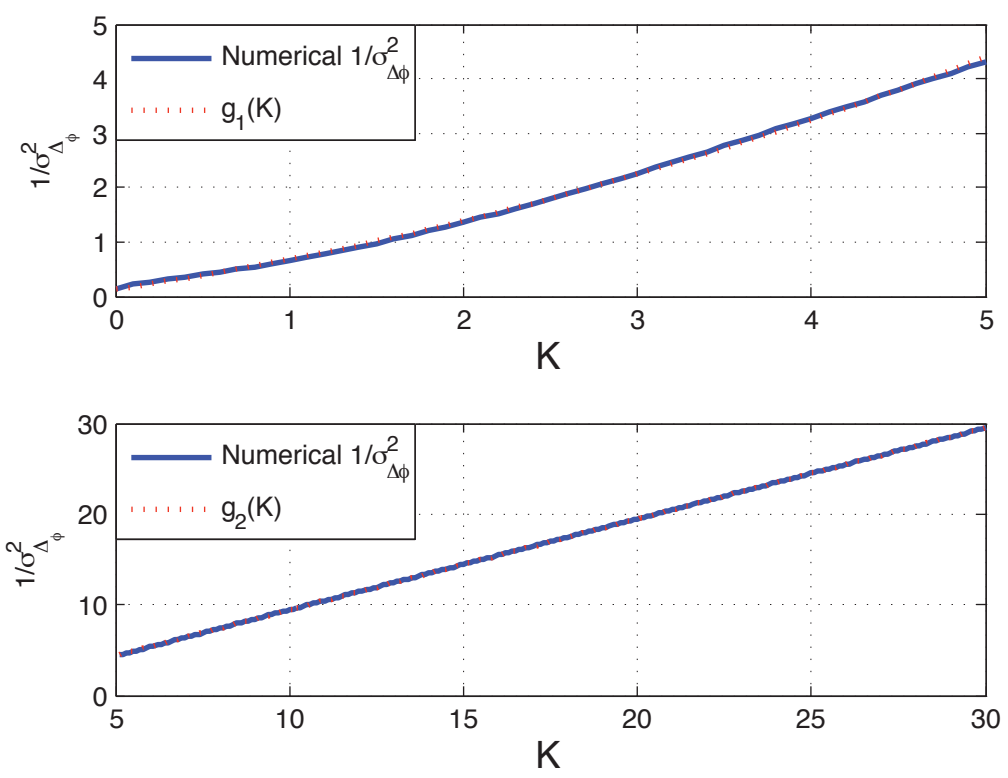

Figure 4.4: Polynomial least square fitting of $g(K)$.

it is obtained

$$
a=0.0738, b=0.4842, c=0.1256, \beta=1.004, \gamma=-0.6121
$$

The approximation $g_{1}(K)$ and $g_{2}(K)$ of $g(K)$ are also shown in Figure 4.4. After solving the quadratic equation and the linear equation resulting from approximating $g(K)$ with $g_{1}(K)$ and $g_{2}(K)$, the estimation $\hat{K}$ of $K$ corresponds to:

$$
\begin{aligned}
& \text { When } \sigma_{\Delta \phi}^{2}<0.232, \quad \hat{K}=\frac{1 / \sigma_{\Delta \phi}^{2}-\gamma}{\beta} \\
& \text { When } \sigma_{\Delta \phi}^{2}>0.232, \quad \hat{K}=\frac{-b+\sqrt{b^{2}-4 a\left(c-1 / \sigma_{\Delta \phi}^{2}\right)}}{2 a}
\end{aligned}
$$

Thus, a one-to-one mapping between $\sigma_{\Delta \phi}^{2}$ and $K$ is maintained, in other words, $K$ can be found given $\sigma_{\Delta \phi}^{2}$. 


\subsection{NLOS identification based on estimated Rician $K$-factor}

In the mobile communication, the Rician $K$-factor is not constant but varies from one location to another due to channel variations. Therefore, $K$ can be modeled by a random variable conditioned on LOS and NLOS with the conditional PDFs $f(K \mid \operatorname{LOS})$ and $f(K \mid$ NLOS $)$, respectively, and

$H_{0} \longrightarrow$ NLOS condition: $K \sim f(K \mid$ NLOS $)$ with probability $P\left(H_{0}\right)$

$H_{1} \longrightarrow$ LOS condition: $K \sim f(K \mid \mathrm{LOS})$ with probability $P\left(H_{1}\right)$

Now, $f(K \mid \mathrm{NLOS})$ and $f(K \mid \mathrm{LOS})$ are investigated. Let $P_{\mathrm{r}}^{\mathrm{LOS}}$ denote the received signal power in $\mathrm{dB}$ via the LOS path,

$$
P_{\mathrm{r}}^{\mathrm{LOS}}=P_{\mathrm{t}}-L^{\mathrm{LOS}}
$$

where $P_{\mathrm{t}}$ is the transmitted signal power in $\mathrm{dB}$ and $L^{\mathrm{LOS}}$ is the path loss for the LOS path only. Let $P_{\mathrm{r}}^{\mathrm{NLOS}}$ denote the received signal power in $\mathrm{dB}$ in NLOS scenario,

$$
P_{\mathrm{r}}^{\mathrm{NLOS}}=P_{\mathrm{t}}-L^{\mathrm{NLOS}}-L_{\mathrm{sh}}
$$

where $L^{\mathrm{NLOS}}$ denotes the path loss for the NLOS scenario and $L_{\mathrm{sh}}$ accounts for shadowing 
effect in $\mathrm{dB} . L_{\mathrm{sh}}$ is a random variable and usually modeled by normal distribution with zero mean and standard deviation $\sigma_{\text {sh }}$

$$
L_{\mathrm{sh}} \sim N\left(0, \sigma_{\mathrm{sh}}^{2}\right), \quad f\left(L_{\mathrm{sh}}\right)=\frac{1}{\sqrt{2 \pi} \sigma_{\mathrm{sh}}} \exp \left(\frac{-L_{\mathrm{sh}}^{2}}{2 \sigma_{\mathrm{sh}}^{2}}\right)
$$

Walfisch-Ikegami (WI) [65] path loss model is used, because it distinguishes between LOS and NLOS propagation. This model is suitable for medium city, suburban centers and metropolitan centers. For LOS condition,

$$
L^{\mathrm{LOS}}=42.6+26 \log d_{\mathrm{km}}+20 \log \left(f_{\mathrm{MHz}}\right)
$$

Here $d_{\mathrm{km}}$ corresponds to the LOS distance of transmitters and receivers in $\mathrm{km}$, and $f_{\mathrm{MHz}}$ is the carrier frequency in MHz. For NLOS condition, an obstruction between the transmitter and the receiver greatly attenuates the LOS signal. In this case, $L^{\mathrm{LOS}}$ in (4.8) is modeled by

$$
L_{\mathrm{pe}}^{\mathrm{LOS}}=42.6+26 \log d_{\mathrm{km}}+20 \log \left(f_{\mathrm{MHz}}\right)+L_{\mathrm{pe}}
$$

where $L_{\mathrm{pe}}$ is the penetration loss.

The authors in [66] have studied the building penetration power loss, which is lognormal distributed with mean $8.1 \mathrm{~dB}$ and standard deviation 6.2dB in urban area. In [66], one time penetration, i.e., from outside to inside, is considered. However, here the penetration loss of passing through buildings is considered. Therefore, the mean and standard deviation of 
this $L_{\mathrm{pe}}$ are doubled. Thus, $L_{\mathrm{pe}}$ is considered normal with mean $\mu_{\mathrm{pe}}=16.2 \mathrm{~dB}$ and standard deviation $\sigma_{\mathrm{pe}}=12.4 \mathrm{~dB}$, i.e., $L_{\mathrm{pe}} \sim N\left(\mu_{\mathrm{pe}}, \sigma_{\mathrm{pe}}^{2}\right)$

For $L^{\mathrm{NLOS}}$ in (4.9), according to WI model,

$$
L^{\mathrm{NLOS}}=A_{\mathrm{nlos}}+38 \log d_{\mathrm{km}}
$$

where $A_{\text {nlos }}$ is a parameter that varies with the signal carrier frequency, the transmitter and receiver antenna heights, the structure of buildings and roads, and the street orientation relative to the direct radio path, and $d_{\mathrm{km}}$ was defined in (4.11). Now using (4.8), (4.9) and (4.12), the LOS $K$-factor in $\mathrm{dB}$, i.e., $K_{\mathrm{dB}}^{\mathrm{LOS}}$ has the expression,

$$
\begin{aligned}
K_{\mathrm{dB}}^{\mathrm{LOS}} & =P_{\mathrm{r}}^{\mathrm{LOS}}-P_{\mathrm{r}}^{\mathrm{NLOS}} \\
& =L_{\mathrm{sh}}+L^{\mathrm{NLOS}}-L^{\mathrm{LOS}}
\end{aligned}
$$

and, in NLOS situations, $K_{\mathrm{dB}}^{\mathrm{NLOS}}$ is

$$
K_{\mathrm{dB}}^{\mathrm{NLOS}}=L_{\mathrm{sh}}-L_{\mathrm{pe}}+L^{\mathrm{NLOS}}-L^{\mathrm{LOS}}
$$

Observing (4.11) and (4.13), it is found that $L^{\mathrm{NLOS}}-L^{\mathrm{LOS}}$ contains the term $12 \log d_{\mathrm{km}}$, which suggests that the mean of $K_{\mathrm{dB}}^{\mathrm{LOS}}$ increases with distance. Based on the above derivations and due to the fact that $L_{\mathrm{sh}}$ and $L_{\mathrm{pe}}$ are Gaussian, both $K_{\mathrm{dB}}^{\mathrm{LOS}}$ and $K_{\mathrm{dB}}^{\mathrm{NLOS}}$ are Gaussian distributed. $K_{\mathrm{dB}}^{\mathrm{LOS}}$ has standard deviation $\sigma_{\mathrm{sh}}$ and mean $L^{\mathrm{NLOS}}-L^{\mathrm{LOS}}$, and $K_{\mathrm{dB}}^{\mathrm{NLOS}}$ has 
standard deviation $\sqrt{\sigma_{\mathrm{sh}}^{2}+\sigma_{\mathrm{pe}}^{2}}$ and mean $L^{\mathrm{NLOS}}-L^{\mathrm{LOS}}-\mu_{\mathrm{pe}}$. Two empirical $K_{\mathrm{dB}}^{\mathrm{LOS}}$ distributions given in [67] and [68] both agree with the normal distribution.

As discussed in (4.11)-(4.13), the parameters that represent $K$ statistics should be tuned well, because those parameters are functions of antenna heights, the carrier frequency, the LOS distance, and etc.. Those parameters can be obtained by measuring the path loss in a practical system.

Two types of hypotheses test will be studied in the following, depending on the availability of $P\left(H_{1}\right)$.

\subsubsection{Known Prior Probability $P\left(H_{1}\right)$}

The prior probability $P\left(H_{1}\right)$ can be learned from previous LOS identification records, or it is obtained from the environmental based empirical LOS likelihood formula, which is used by the European IST project WINNER [69].

When the prior probability $P\left(H_{1}\right)$ is known, a Maximum A-Posteriori (MAP) detection can be formed to achieve the minimum error probability. Here, the decision rules are

\footnotetext{
Decide NLOS if $f\left(K_{\mathrm{dB}}^{\mathrm{NLOS}}\right) P\left(H_{0}\right)>f\left(K_{\mathrm{dB}}^{\mathrm{LOS}}\right) P\left(H_{1}\right)$

Decide LOS if $f\left(K_{\mathrm{dB}}^{\mathrm{NLOS}}\right) P\left(H_{0}\right)<f\left(K_{\mathrm{dB}}^{\mathrm{LOS}}\right) P\left(H_{1}\right)$
} 
Decide NLOS if $K<K_{\mathrm{th}}$

Decide LOS if $K>K_{\text {th }}$

$K_{\mathrm{th}}$ can be obtained by finding the intersection of $f\left(K_{\mathrm{dB}}^{\mathrm{NLOS}}\right) P\left(H_{0}\right)$ and $f\left(K_{\mathrm{dB}}^{\mathrm{LOS}}\right) P\left(H_{1}\right)$. Let $\sigma_{1}$ and $\mu_{1}$ be the standard deviation and mean of $K_{\mathrm{dB}}^{\mathrm{LOS}}$, and let $\sigma_{0}$ and $\mu_{0}$ be the standard deviation and mean of $K_{\mathrm{dB}}^{\mathrm{NLOS}}$. Equating $f\left(K_{\mathrm{dB}}^{\mathrm{NLOS}}=K_{\mathrm{th}}\right) P\left(H_{0}\right)$ and $f\left(K_{\mathrm{dB}}^{\mathrm{LOS}}=K_{\mathrm{th}}\right) P\left(H_{1}\right)$, the quadratic equation of $K_{\text {th }}$ can be solved,

$K_{\mathrm{th}}=\frac{\left(\sigma_{0}^{2} \mu_{1}-\sigma_{1}^{2} \mu_{0}\right)-\sqrt{\left(\left(\sigma_{0}^{2} \mu_{1}-\sigma_{1}^{2} \mu_{0}\right)^{2}-\left(\sigma_{0}^{2}-\sigma_{1}^{2}\right)\left(\sigma_{0}^{2} \mu_{1}^{2}-\sigma_{1}^{2} \mu_{0}^{2}-\sigma_{1}^{2} \sigma_{0}^{2} \ln \frac{\sigma_{0} P\left(H_{1}\right)}{\sigma_{1} P\left(H_{0}\right)}\right)\right.}}{\left(\sigma_{0}^{2}-\sigma_{1}^{2}\right)}$

The other solution is larger and omitted because $K_{\mathrm{dB}}^{\mathrm{NLOS}}$ rarely accepts large values.

The detection probability of NLOS condition is defined by

$$
P_{\mathrm{D}}=\int_{-\infty}^{K_{\mathrm{th}}} f\left(K_{\mathrm{dB}}^{\mathrm{NLOS}}=k\right) d k=1-Q\left(\left(K_{\mathrm{th}}-\mu_{0}\right) / \sigma_{0}\right)
$$

and the false alarm probability of NLOS condition is defined by

$$
P_{\mathrm{F}}=\int_{-\infty}^{K_{\mathrm{th}}} f\left(K_{\mathrm{dB}}^{\mathrm{LOS}}=k\right) d k=1-Q\left(\left(K_{\mathrm{th}}-\mu_{1}\right) / \sigma_{1}\right)
$$




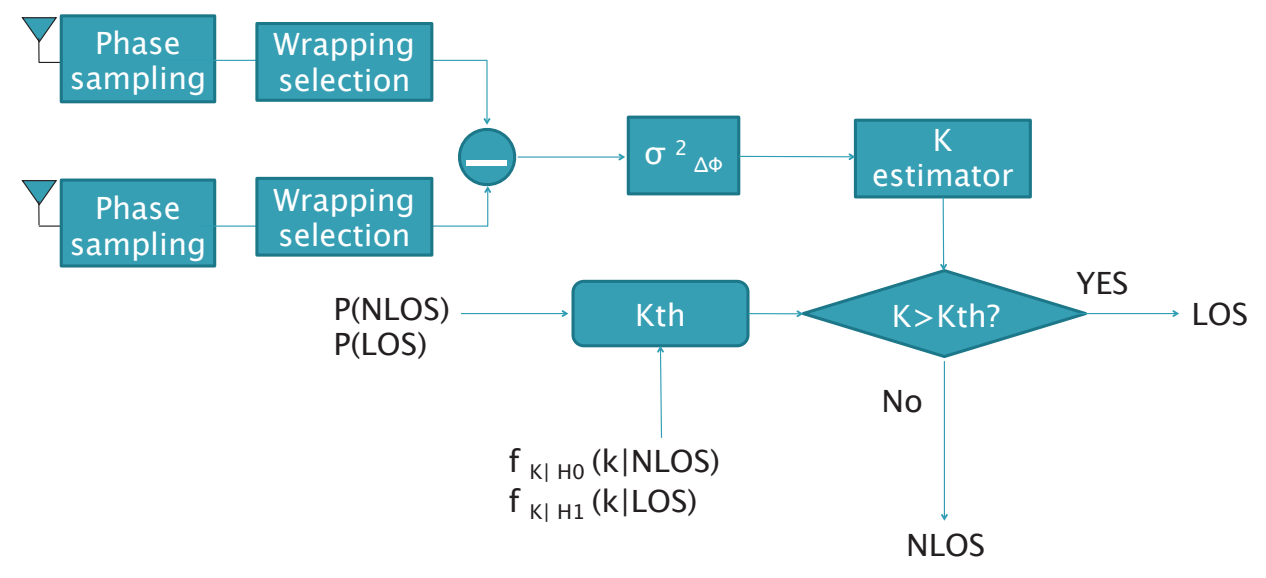

Figure 4.5: Block diagram of NLOS identification technique based on phase difference across two antenna elements

Therefore the error probability is $P_{\mathrm{E}}=\left(1-P_{\mathrm{D}}\right) P\left(H_{1}\right)+P_{\mathrm{F}} P\left(H_{0}\right)$.

\subsubsection{Unknown Prior Probability $P\left(H_{1}\right)$}

In some cases, it is difficult to evaluate the prior probability $P\left(H_{1}\right)$. In those scenarios, the Neyman-Pearson (NP) test can be used [70]. NP test aims to maximize $P_{\mathrm{D}}$ by constraining $P_{\mathrm{F}}=\alpha$. The procedure follows,

1. Set the false alarm probability $P_{\mathrm{F}}=\alpha$.

2. Solve the threshold $K_{\mathrm{th}}$ from $\alpha=\int_{K_{\mathrm{th}}}^{\infty} f\left(K_{\mathrm{dB}}^{\mathrm{NLOS}}=k\right) d k$.

3. Decide NLOS or LOS by rules given in (4.16).

The overall algorithm of NLOS identification technique based on phase difference is illustrated in Figure 4.5. In the figure, the wrapping selection algorithm is given above (4.3); $K$ 
estimator is shown in (4.7); the distributions of $K$ under NLOS condition $f_{K \mid H_{0}}(k \mid N L O S)$ and $K$ under LOS condition $f_{K \mid H_{1}}(k \mid L O S)$ are derived in Section 4.3; $K_{t h}$ is computed by (4.17) according to MAP detection rule or is computed from a given value of $P_{F}$ according to NP test.

The estimated $\mathrm{K}$ is derived from the phase difference variance across two antenna elements. In order to calculate this variance, a large number of independent phase difference samples should be collected in time domain. The time difference between any two consecutive phase difference sample should be selected large enough (for example greater than the channel coherence time) to ensure the independence of the phase differences. If more antenna elements are incorporated, independent phase difference variances across any two consecutive antenna element pair can be created. In other words, independent phase difference samples can be created in the space domain as well. Thus, if using two antenna elements $T$ second was required to improve the localization performance, using $M$ antenna elements this can be reduced to $T /(M-1)$. Thus, increasing the number of antenna elements, the phase difference variance can be computed in a shorter time period.

\subsection{Uncorrelated phase verification}

This section investigates the phase correlation in two typical propagation environments: circular scattering model [47] and elliptic scattering model [71]. Via simulation, the phase uncorrelation assumption is justified and the influence of environment parameters are stud- 


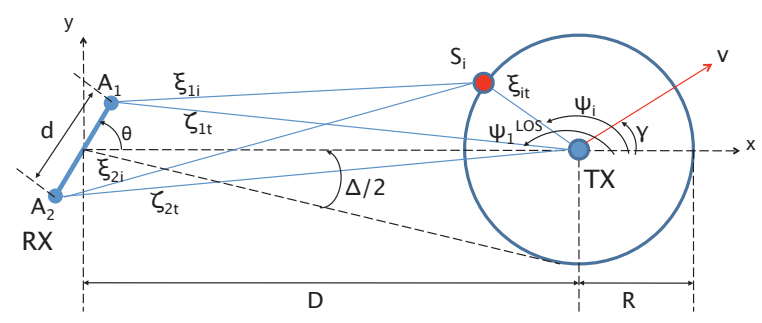

(a)

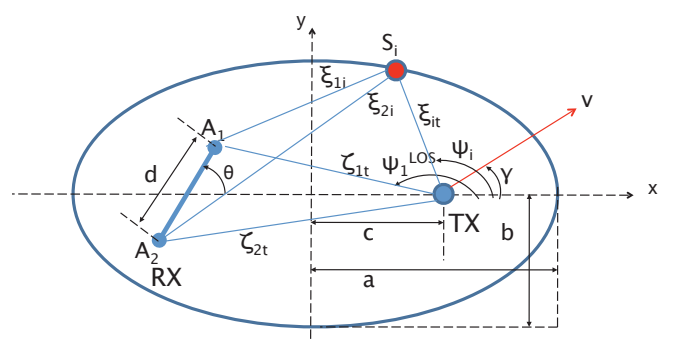

(b)

Figure 4.6: Geometrical configuration of (a) circular scattering (b) elliptic scattering.

ied.

In (4.3), $\operatorname{cov}\left(\Delta \phi_{1} \Delta \phi_{2}\right)$ might not be zero in reality. By intuition, as shown in Figure 4.2, the correlation between $\Delta \phi_{1}$ and $\Delta \phi_{2}$ is determined by the dependency of $r_{\mathrm{DIF} 1}$ and $r_{\mathrm{DIF} 2}$, as well as the value of $K$-factor.

The geometry of scatterers has a great impact on the correlation of the received signals in multi-antenna systems. The geometrical setting of the circular and elliptic scattering is presented in Figure 4.6. The receiver (RX) is equipped with two antennas and is set to detect the LOS/NLOS condition of the omni-directional transmitter (TX). Circular model represents environments where the antenna height of RX is relatively large and the antenna height of TX is small. In this case, signal scattering from locations near the RX would be ignored. However, TX is assumed surrounded by a circle of scatterers. Elliptic model represents scenarios where antenna heights of both RX and TX are low, and therefore the scattering near the RX is as likely as the scattering near the TX.

Now, expressions of the received signals are derived for both models. In Figure 4.6 (a), 
scatterers lie on a ring with radius $R$ and the TX is at the center of the ring. The $i$ th scatterer is represented by $S_{i}, D$ is the distance between $\mathrm{RX}$ and TX, $\Delta$ is the angle through which the RX receives signals, $\theta$ is the DOA of LOS signals and $d$ is the antenna spacing. This model takes into account the Doppler effect, which is a result of TX mobility. The motion of TX is characterized by its speed $v$ and the direction of motion $\gamma$.

Here, the power of transmitted signal is assumed to be unity and the power of the received signal via LOS or diffused directions does not change during the time over which phase samples are taken. Using Figure 4.6, the expressions for the diffusive and the LOS components of the received signal via Antenna 1 are

$$
\begin{aligned}
& r_{\mathrm{DIF} 1}=\sqrt{\frac{1}{K+1}} \frac{1}{\sqrt{N}} \sum_{i=1}^{N} \exp \left\{j \varphi_{i}-j \frac{2 \pi}{\lambda}\left(\xi_{1 i}+\xi_{i t}\right)+j 2 \pi f_{\mathrm{D}}\left(\cos \left(\psi_{i}-\gamma\right)\right) t\right\} \\
& r_{\mathrm{LOS} 1}=\sqrt{\frac{K}{K+1}} \exp \left\{-\frac{j 2 \pi}{\lambda} \zeta_{1 t}+j 2 \pi f_{\mathrm{D}}\left(\cos \left(\psi_{1}^{\mathrm{LOS}}-\gamma\right) t\right)\right\}
\end{aligned}
$$

In (4.20) and (4.21), $K$ represents the power ratio of LOS component and diffusive component, $N$ is the number of scatterers around the TX, $\varphi_{i}$ denotes the phase shift introduced by the $i$ th scatterer, $\xi_{1 i}$ and $\xi_{i t}$ are the distances from Antenna 1 to the TX via scatterer $S_{i}$, as shown in Figure 4.6 (a), $\psi_{i}$ is the angle of arrival (AOA) of the wave traveling from the scatterer $S_{i}$ toward the TX, $\lambda$ is the wavelength, $j^{2}=-1, f_{\mathrm{D}}=v / \lambda$ is the maximum Doppler shift, and finally, $\zeta_{1 t}$ and $\psi_{1}^{\mathrm{LOS}}$ denote the length and the direction of the LOS path between the RX and the TX. The DIF and LOS components $r_{\mathrm{DIF} 2}$ and $r_{\mathrm{LOS} 2}$ for the received signal via Antenna 2 can be similarly computed. 
For sampling purpose, the trajectory of the received signal is recorded. The position of the scatterers is held constant over the duration in which the TX travels a distance of 5 meters. At the end of the 5-meter, the scatters are returned to their original position with respect to the TX. At each 5-meter interval, random phases $\varphi_{i}$ are assigned to the scatterers.

Figure 4.6 (b) shows the elliptic model. The RX and the TX are located at the foci and $a$ denotes the semimajor axis, $b$ denotes the semiminor axis and $c=\sqrt{a^{2}-b^{2}}$. The definitions of all other parameters are the same as those in Figure 4.6 (a).

The phase and signal correlation are investigated by evaluating their correlation coefficients $\operatorname{coeff}\left(\Delta \phi_{1}, \Delta \phi_{2}\right)$ and $\operatorname{coeff}\left(r_{\mathrm{DIF} 1}, r_{\mathrm{DIF} 2}\right)$ via simulations. The correlation coefficients are defined as:

$$
\operatorname{coeff}\left(\Delta \phi_{1}, \Delta \phi_{2}\right)=\frac{\operatorname{cov}\left(\Delta \phi_{1}, \Delta \phi_{2}\right)}{\sigma_{\Delta \phi_{1}} \sigma_{\Delta \phi_{2}}}
$$

where $\sigma$ denotes the standard deviation. The correlation coefficients coeff $\left(r_{\mathrm{DIF} 1}, r_{\mathrm{DIF} 2}\right)$ is similarly defined. In addition, the variance of $\Delta \phi$ in (4.2), $\sigma_{\Delta \phi}^{2}$ is evaluated to study the impact of the phase correlation on the estimation of $K$ when the parameters vary. First, the simulation results for the circular model are presented. The AOA of LOS signals at the $\mathrm{RX}, \theta$, and the moving direction of TX, $\gamma$ take on values according to independent uniform distribution in $[0,2 \pi]$. There are 20 omni-directional reflectors uniformly distributed along the ring. Samples are taken per $0.1 \lambda$ and the total number of samples is 500 . In addition, $\lambda=0.1 \mathrm{~m}, v=10 \mathrm{~m} / \mathrm{s}, R=50 \mathrm{~m}$. 


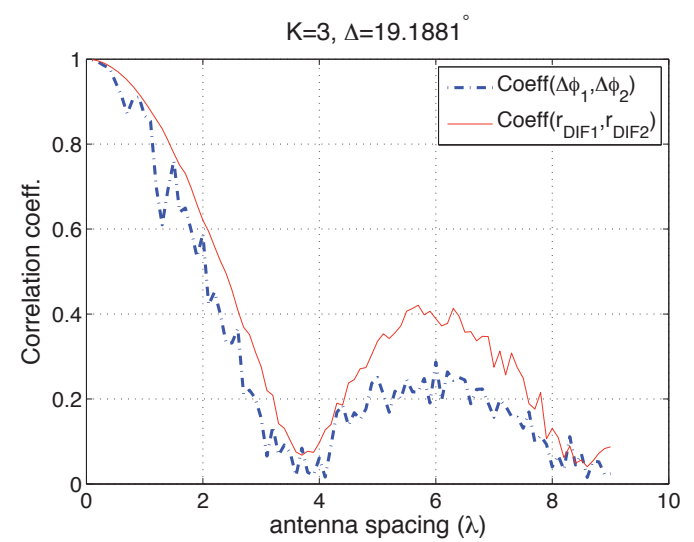

(a)

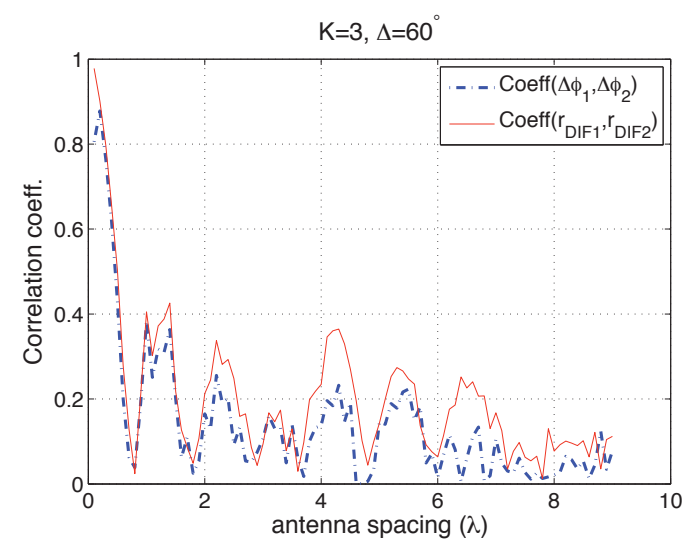

(b)

Figure 4.7: Circular model: $\operatorname{coeff}\left(\Delta \phi_{1}, \Delta \phi_{2}\right)$ and coeff $\left(r_{\mathrm{DIF} 1}, r_{\mathrm{DIF} 2}\right)$ versus antenna spacing for (a) $\Delta=19.2^{\circ}$. (b) $\Delta=60^{\circ}$.

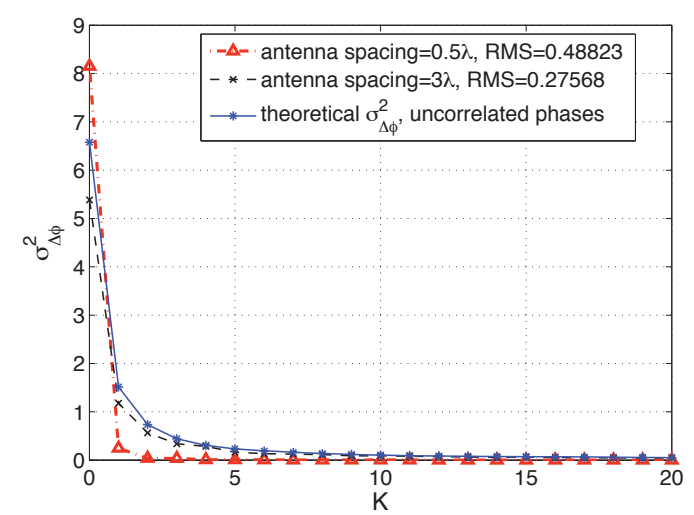

(a)

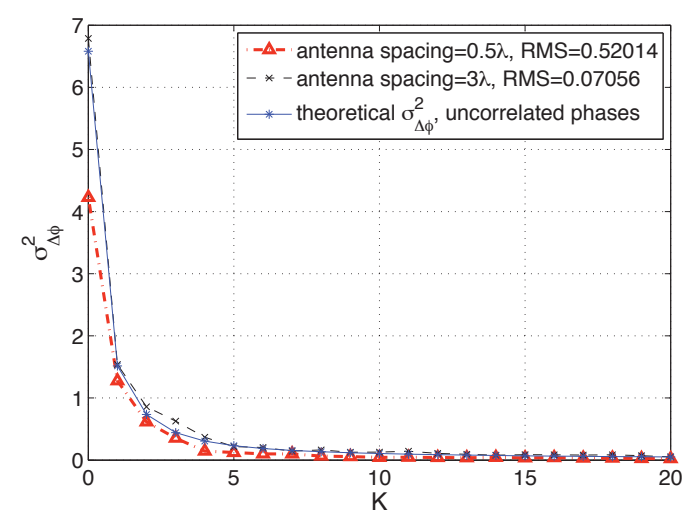

(b)

Figure 4.8: Circular model: comparison of simulated and theoretical $\sigma_{\Delta \phi}^{2}$ for (a) $\Delta=19.2^{\circ}$. (b) $\Delta=60^{\circ}$.

Figure 4.7 shows the phase and signal correlation versus antenna spacings when $\Delta=19.2^{\circ}$ and $\Delta=60^{\circ}$. Comparing the main lobe of the correlation curves, the correlation in Figure 4.7(a) decreases more slowly than that in Figure 4.7(b) . These curves confirm that when the separation of antenna elements is high enough, the phase difference correlation is reasonably small. 


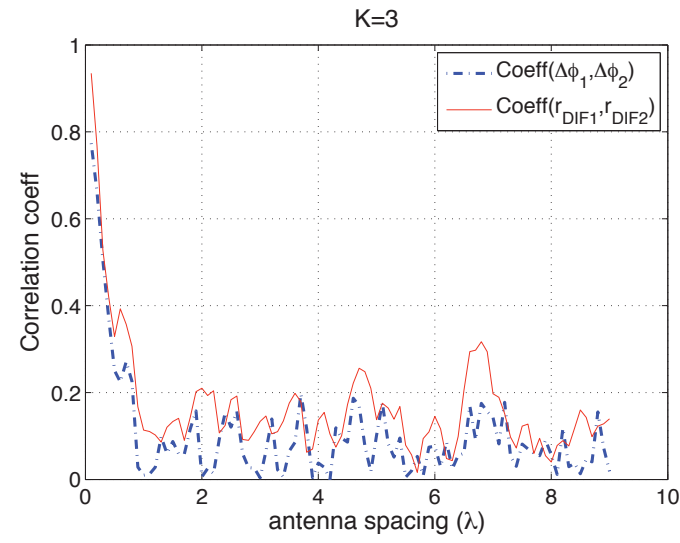

(a)

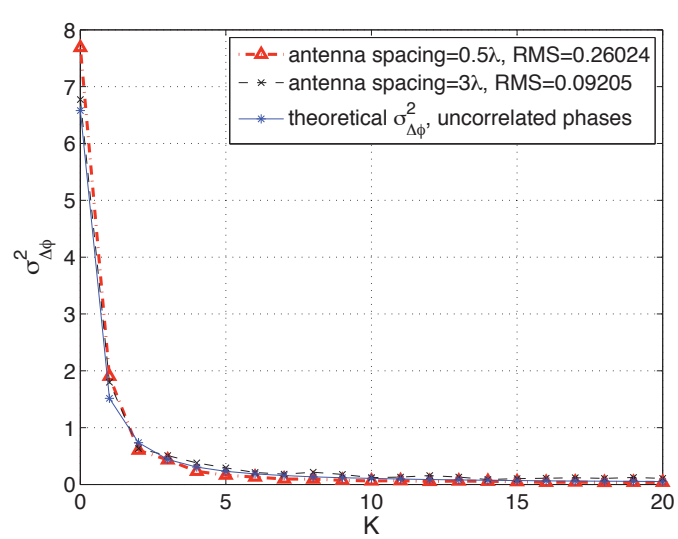

(b)

Figure 4.9: Elliptic model: (a) phase and signal correlation coefficients versus antenna spacing (b) comparison of simulated and theoretical $\sigma_{\Delta \phi}^{2}$.

Figure 4.8 compares the simulated $\sigma_{\Delta \phi}^{2}$ with the theoretical $\sigma_{\Delta \phi}^{2}$ of uncorrelated phases, given by (4.5). The performance is measured by the root mean square error (RMS) of the difference between the simulated and theoretical curves. In both Figure 4.8(a) and (b), the RMSs of $0.5 \lambda$ antenna spacing are greater than those of $3 \lambda$ antenna spacing, because the phase correlation drops significantly for $3 \lambda$ antenna spacing. The values of $\sigma_{\Delta \phi}^{2}$ at $K=1$ for both antenna spacings, shown in Figure 4.8(b), fits the theoretical results better than those shown in Figure 4.8(a). This outcome is consistent with our observations in Figure 4.7 which confirms that the correlation coefficients for $0.5 \lambda$ and $3 \lambda$ spacing at larger opening angle $\Delta=60^{\circ}$ is smaller.

Now, the results of the elliptic model is investigated. Simulation parameters are similar to those listed for the circular model except $a=100 \mathrm{~m}$ and $b=50 \mathrm{~m}$. The results for antenna spacing $0.5 \lambda$ and $3 \lambda$ are compared in Figure $4.9(b)$, where their performance in estimating $K$ is acceptable. This is a result of low phase correlation at $0.5 \lambda \& 3 \lambda$ antenna spacing 


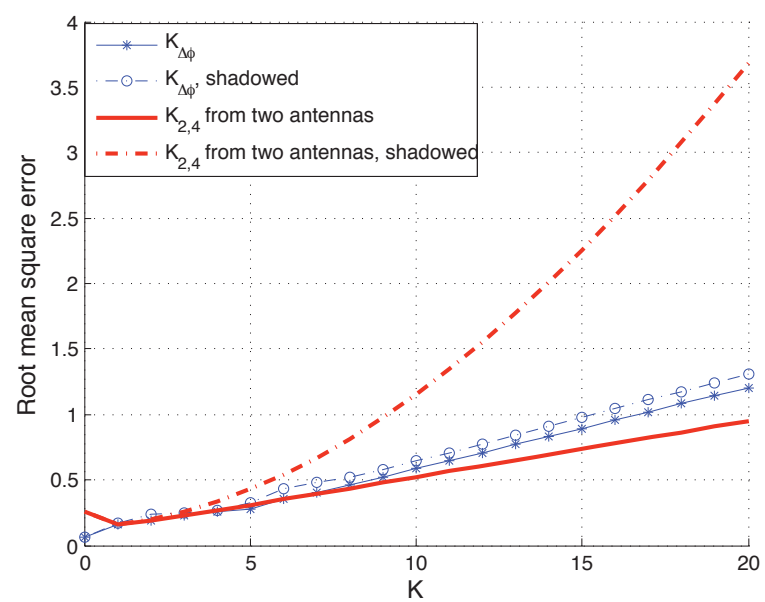

(a)

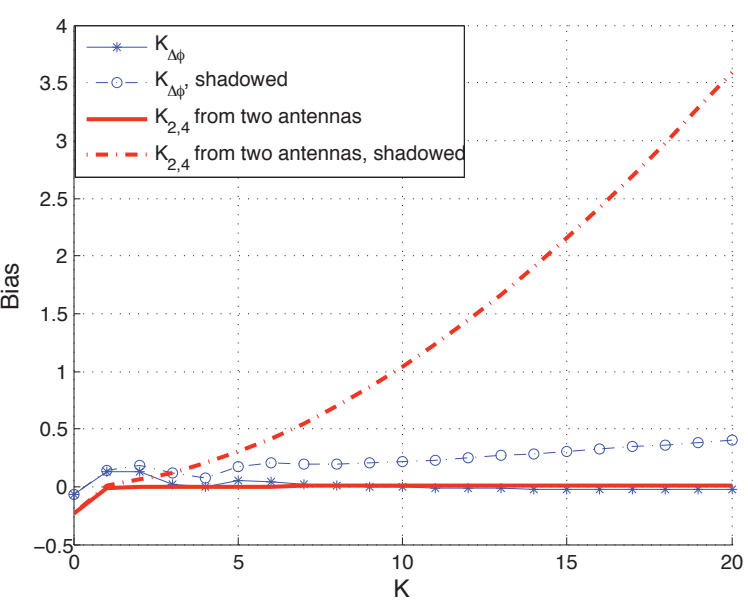

(b)

Figure 4.10: Performance comparison of $\hat{K}_{\Delta \phi}$ and $\hat{K}_{2,4}$ estimators with a sample size of $N=500$ : (a) Root mean square error (b) Bias.

(Figure 4.9(a)). In the elliptic model, the RX receives signal from all directions which contributes to the low phase correlation, while in the circular model, the RX receives signal just from an opening angle of $\Delta$. It is suggested in Figure 4.9(b) that in elliptic model, $\lambda / 2$ antenna spacing is sufficient for $K$ estimation.

\subsection{Simulation results}

Simulations are conducted to investigate the performance of $K$ estimator introduced in Section 4.2 for NLOS identification. And the probability-of-detection and the probabilityof-false alarm defined in (4.18) and (4.19) are studied as well.

Figure 4.10 compares the performance of the $\hat{K}_{\Delta \phi}$ estimator and the performance of $\hat{K}_{2,4}$ for constant LOS component and shadowed LOS component. $\hat{K}_{\Delta \phi}$ is the estimation based on phase difference introduced in (4.7) and $\hat{K}_{2,4}$ is based on the second and fourth moments 
of the received signal envelope [72], corresponding to

$$
\hat{K}_{2,4}=\frac{-2 \mathrm{E}^{2}\left(R^{2}\right)+\mathrm{E}\left(R^{4}\right)-\mathrm{E}^{2}\left(R^{2}\right) \sqrt{2 \mathrm{E}^{2}\left(R^{2}\right)-\mathrm{E}\left(R^{4}\right)}}{\mathrm{E}^{2}\left(R^{2}\right)-\mathrm{E}\left(R^{4}\right)}
$$

where $R$ denotes the received signal envelope, $\mathrm{E}(\cdot)$ denotes expectation, $\mathrm{E}\left(R^{2}\right)$ is the second moment and $\mathrm{E}\left(R^{4}\right)$ is the fourth moment. It is noted that the amplitude of LOS component would be affected by shadowing characterized by Nakagami distribution [73]. The root mean square error (RMSE) in Figure 4.10(a) is defined as $\sqrt{\mathrm{E}(K-\hat{K})^{2}}$, where $K$ is the true value and $\hat{K}$ is the estimated value. The bias in Figure $4.10(\mathrm{~b})$ is defined as $\mathrm{E}(K-\hat{K})$.

Simulation parameters are as follows: in Figure 4.2, $r_{\mathrm{DIF} 1}$ and $r_{\mathrm{DIF} 2}$ are independent complex Gaussian variables; $\phi_{\mathrm{LOS} 1}$ and $2 \pi d \cos \theta / \lambda$ are deterministic but unknown, taking on values from a uniform distribution in the range $[-\pi, \pi]$; number of samples $N=500$; for constant LOS case, $r_{\mathrm{LOS} 1,2}$ is constant and for shadowed LOS case, $\left|r_{\mathrm{LOS} 1,2}\right|$ is a Nakagami distributed random variable following the PDF given as

$$
P\left(\left|r_{\mathrm{LOS} 1,2}\right|=r\right)=\frac{2 m^{m} r^{2 m-1}}{\Gamma(m) P_{r}^{m}} \exp \left[\frac{-m r^{2}}{P_{r}}\right]
$$

where $P_{r}=\mathrm{E}\left(\left|r_{\mathrm{LOS} 1,2}\right|^{2}\right)$ is the average power of the LOS component. The Nakagami distribution is parameterized by $P_{r}$ and the fading parameter $m=50$. The larger $m$ is, the less shadowing is on the LOS component. $\Delta \phi$ is calculated by (4.2). In addition, the wrapping in (4.2) is maintained via algorithm introduced above (4.3) in section 4.2. 


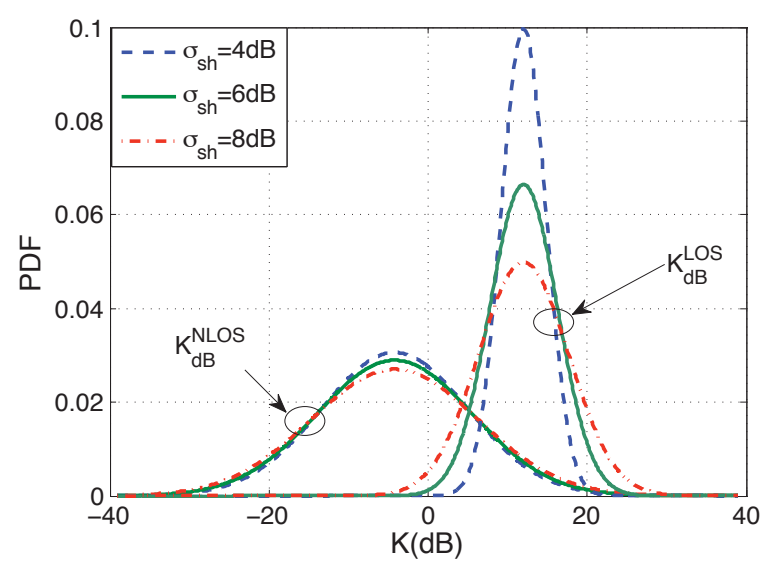

(a)

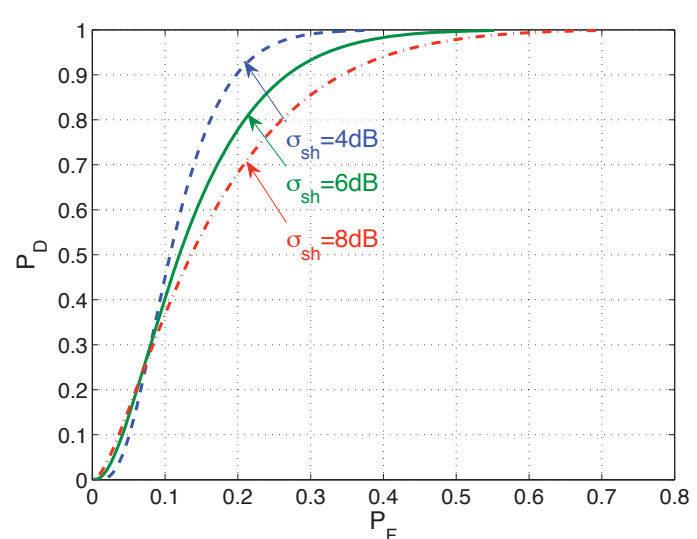

(b)

Figure 4.11: For $\sigma_{\mathrm{sh}}=4 \mathrm{~dB}, 6 \mathrm{~dB}, 8 \mathrm{~dB}$ (a) PDF of $K_{\mathrm{dB}}^{\mathrm{NLOS}}$ and $K_{\mathrm{dB}}^{\mathrm{LOS}}$ (b) probabilityof-detection $P_{\mathrm{D}}$ V.S. probability-of-false alarm $P_{\mathrm{F}}$

It is observed in Figure 4.10(a) that for the case of constant LOS, $\hat{K}_{2,4}$ has a lower estimation error than $\hat{K}_{\Delta \phi}$ when $K \geq 7$. However, when the LOS component is subject to random shadowing, the RMSE of $\hat{K}_{2,4}$ is much higher than that of $\hat{K}_{\Delta \phi}$. This is because a perturbation on LOS component makes the envelope distribution deviating from Rician which is required in deriving the formula of $\hat{K}_{2,4}$ (4.23). Although the formula of $\hat{K}_{\Delta \phi}(4.7)$ is based on a constant LOS component too, the impact of the LOS component perturbation on $\hat{K}_{\Delta \phi}$ is not as severe as that on $\hat{K}_{2,4}$. Compared to the constant LOS results, it is clearly shown in Figure 4.10(a) that the RMSE of $\hat{K}_{2,4}$ increases significantly (due to a big bias shown in Figure 4.10(b)) while the RMSE of $\hat{K}_{\Delta \phi}$ increases slightly.

This observation suggests that compared to envelope based $K$ estimators, $K_{\Delta \phi}$ estimator is more robust to amplitude variation of LOS component.

Now, the performance of NLOS identification based on $K_{\Delta \phi}$ estimator is evaluated. The 
Table 4.1: Comparison of $K_{\mathrm{th}}, P_{\mathrm{F}}$ and $P_{\mathrm{D}}$ for various $\sigma_{\mathrm{sh}}$

\begin{tabular}{|c|c|c|c|}
\hline & $K_{\text {th }}$ & $P_{\mathrm{F}}$ & $P_{\mathrm{D}}$ \\
\hline$\sigma_{\mathrm{sh}}=4 \mathrm{~dB}$ & $6.54 \mathrm{~dB}$ & 0.0863 & 0.7952 \\
\hline$\sigma_{\mathrm{sh}}=6 \mathrm{~dB}$ & $5.17 \mathrm{~dB}$ & 0.1276 & 0.7519 \\
\hline$\sigma_{\mathrm{sh}}=8 \mathrm{~dB}$ & $4.24 \mathrm{~dB}$ & 0.1662 & 0.7164 \\
\hline
\end{tabular}

carrier frequency is assumed to be $1.9 \mathrm{GHz} . A_{\text {nlos }}$ in (4.13) is set at $132 \mathrm{~dB}$ based on some typical building and road parameters, $d_{\mathrm{km}}=0.1 \mathrm{~km}$, and the mean of $K_{\mathrm{dB}}^{\mathrm{LOS}}$ is computed to be $12 \mathrm{~dB}$. The distribution of $K_{\mathrm{dB}}^{\mathrm{LOS}}$ and $K_{\mathrm{dB}}^{\mathrm{NLOS}}$ is plotted in Figure 4.11(a) for shadowing standard deviation $\sigma_{\mathrm{sh}}=4 \mathrm{~dB}, 5 \mathrm{~dB}, 6 \mathrm{~dB}$. It is observed that as $\sigma_{\mathrm{sh}}$ increases, the intersection area of $K_{\mathrm{dB}}^{\mathrm{NLOS}}$ and $K_{\mathrm{dB}}^{\mathrm{LOS}}$ increases, along with which the probability-of-error will increase. When the LOS condition likelihood $P\left(H_{1}\right)=0.5$, the MAP detection rule is applied. Plugging the mean and standard deviation into (4.17)-(4.19), the threshold $K_{\text {th }}$, the detection probability of NLOS, $P_{\mathrm{D}}$ and the false alarm probability of NLOS, $P_{\mathrm{F}}$ are summarized in Table 4.1.

In Neyman Pearson test, the false alarm rate is set to a desirable value, and then the threshold separating LOS and NLOS can be found. Using (4.18), the detection probability $P_{\mathrm{D}}$ is evaluated. The relationship of detection probability $P_{\mathrm{D}}$ and false alarm probability $P_{\mathrm{F}}$ is sketched for different values of shadowing variance in Figure 4.11(b). It shows that as $\sigma_{\mathrm{sh}}$ decreases, the curve moves to the upper left region. This means a better identification performance can be attained, because for each fixed value of $P_{F}$, lower $\sigma_{\mathrm{sh}}$ achieves higher $P_{D}$. 


\subsection{Conclusion}

In this chapter, a novel NLOS identification technique is proposed. It extracts the phase difference of two signals received via two antenna elements. The proposed technique assumes uncorrelated phase across the two antenna elements. Simulations depict that this assumption is valid for elliptical distribution of scatterers as long as antenna element spacing exceeds one wavelength. However, for circular distribution of scatterers, antenna element spacing that ensures independency of the signal phase across antenna elements varies with the distance of transmitter and receiver: as the transmitter-receiver distance increases, higher antenna element spacing is required to ensure the independency of phases across two antenna elements.

Thus, the proposed model performs better for elliptical models for which the antenna element spacing needs to be selected low. The elliptical model is a good model when the altitude of both the transmitter and receiver is low. This motivates the application of the proposed technique for near ground sensor networks in urban areas, such as those that could be installed on vehicles for traffic alert and collision avoidance.

Compared to the traditional envelope-based $K$ estimator, the proposed phase differencebased $K$ estimator is more robust when the signal envelope distribution deviates from $\mathrm{Ri}$ cian. For example, when the LOS component is subject to random shadowing, the envelope distribution deviates from Rician. The impact of shadowing variance is investigated. It is 
shown that smaller shadowing variance leads to a better NLOS identification performance. Typically, a variance of $4 \mathrm{~dB}$ leads to 0.7952 probability of identification while a variance of $8 \mathrm{~dB}$ leads to 0.7164 probability of identification. This technique can also be applied to systems with more than two antenna elements. Additional antennas increase the speed of data acquisition process and reduce the identification processing time. 


\section{Chapter 5}

\section{NLOS Identification in Frequency Selective and}

\section{Space Selective radio channels}

This chapter proposes a non-line-of-sight (NLOS) identification technique that exploits space-frequency channel correlation of multi-input multi-output (MIMO) orthogonal frequency division multiplexing (OFDM) systems. Here, space correlation refers to the correlation across antenna elements, and frequency correlation refers to the correlation across subcarriers. In this chapter, metrics based on space-frequency channel correlation are proposed for NLOS identification. The proposed metrics require minimal variation of spatial correlation across different multi-path components. The channel model satisfying this requirement is studied. The probability of detection performance of the new NLOS identification method is investigated. 


\subsection{Introduction}

In this chapter, we investigate the NLOS identification problem for wireless wide band multi-input multi-output (MIMO) systems. MIMO systems use multiple antenna elements at the transmitter and receiver to maintain high throughput and reliable communication. In wide band systems, orthogonal-frequency-division-multiplexing (OFDM) can significantly reduce the receiver complexity [74]. Therefore, the use of MIMO technology in combination with OFDM, i.e., MIMO-OFDM is an attractive solution for high throughput wireless communications and it is also a promising candidate for location-based services. MIMO-OFDM has already been adopted as the standard for wireless LAN 802.11n[75] and WiMAX 802.16e [76].

We propose a new NLOS identification technique exploiting space-time-frequency channel correlation of MIMO-OFDM systems. The channel correlation of MIMO-OFDM systems can be measured across antenna (space) and across subcarrier (frequency). It is noted that the channel information is available at the receiver in MIMO-OFDM systems and accordingly, the measurement of channel correlation is straight forward. In this chapter, the proposed metrics for NLOS identification are based on space-frequency correlation and they include: (1) the absolute value of subcarrier correlation difference (SCD) and (2) the mean value and (3) the standard deviation (std) of SCD over different transmit and receive antenna combinations. The identification is based on the following observation: 
when the variation of spatial correlation across different multi-path components is minimal, the absolute value of SCD, and the mean value and std of SCD under LOS conditions would be larger than those under NLOS conditions with a large probability. The proposed technique functions assuming microcell channel models. For the channel models fail to meet minimal variation requirement, NLOS identification techniques for MIMO-OFDM systems are investigated in Chapter 6.

The remainder of this chapter is organized as follows. In Section 5.2, we introduce the space-frequency channel correlation model of MIMO-OFDM systems, and define the corresponding metrics: SCD, the mean value and std of SCD. In Section 5.3, we present the performance of NLOS identification using the metrics defined in Section 5.2. Section 5.4 concludes the chapter.

\subsection{Techniques based on space-frequency channel correlation}

\subsubsection{Space-frequency channel correlation}

We consider a wideband MIMO channel with $M_{t}$ and $M_{r}$ transmit and receive antenna elements, respectively. When LOS is available, the multi-path channel impulse response

(CIR) from the $p^{\text {th }}\left(p=1,2, \ldots M_{t}\right)$ transmit antenna to the $q^{\text {th }}\left(q=1,2, \ldots M_{r}\right)$ receive 
antenna is represented by a tapped-delay-line model that corresponds to

$$
h_{L O S}^{p, q}(t)=a_{L O S}^{p, q} \delta\left(t-\tau_{L O S}\right)+\sum_{l=1}^{L} a_{l}^{p, q} \delta\left(t-\tau_{l}\right)
$$

where $a_{L O S}^{p, q}$ is the amplitude coefficient of the LOS path, $\tau_{L O S}$ is the TOA of the LOS path, $a_{l}^{p, q}$ is the amplitude coefficient of the $l^{\text {th }}$ multi-path component, and $\tau_{l}$ is the associated TOA. Let $\varepsilon_{l}^{2}$ be the power of the $l^{\text {th }}$ tap for any transmit-receive antenna combination, i.e., $\varepsilon_{l}^{2}=E\left(\left|a_{l}^{p, q}\right|^{2}\right), \quad p=1,2, \ldots M_{t}, q=1,2, \ldots M_{r}$. Usually, the first tap contains the LOS and some NLOS rays. Thus, $\tau_{1}=\tau_{L O S}$.

Assuming $a_{l}^{p, q}$ is a zero mean Gaussian process, $a_{L O S}^{p, q}$ is a complex number and $\left|a_{L O S}^{p, q}\right|^{2}=$ $\left|a_{L O S}\right|^{2}$, the Rician $K$-factor of the first path is defined as

$$
K \triangleq \frac{\left|a_{L O S}^{p, q}\right|^{2}}{E\left(\left|a_{1}^{p, q}\right|^{2}\right)}=\frac{\left|a_{L O S}\right|^{2}}{\varepsilon_{1}^{2}}
$$

Next, we compute the channel response in the frequency domain. Let the signal bandwidth be $W$ and the total number of subcarriers is $N$, the frequency spacing in OFDM is defined as $\Delta f \triangleq W / N$. Applying Fourier transform to (6.1), the channel frequency response of the 
$n^{\text {th }}$ subcarrier is obtained,

$$
\begin{aligned}
H_{L O S}^{p, q}(n) & =a_{L O S}^{p, q} \exp \left(-j 2 \pi n \Delta f \tau_{L O S}\right) \\
& +\sum_{l=1}^{L} a_{l}^{p, q} \exp \left(-j 2 \pi n \Delta f \tau_{l}\right)
\end{aligned}
$$

where $n=0,1, \ldots N-1$ and $\tau_{L O S}$ can always be normalized to 0 without loss of generality. Then, the normalized correlation of the channel response from the $p_{1}^{t h}$ transmit antenna to the $q_{1}^{\text {th }}$ receive antenna at the $n_{1}^{\text {th }}$ subcarrier and the channel response from the $p_{2}^{\text {th }}$ transmit antenna to the $q_{2}^{\text {th }}$ receive antenna at the $n_{2}^{\text {th }}$ subcarrier is

$$
\begin{aligned}
& R_{L O S}\left(p_{1}, q_{1}, n_{1} ; p_{2}, q_{2}, n_{2}\right) \\
& =E\left\{H_{L O S}^{p_{1}, q_{1}}\left(n_{1}\right)\left[H_{L O S}^{p_{2}, q_{2}}\left(n_{2}\right)\right]^{*}\right\} / E\left\{H_{L O S}^{p_{1}, q_{1}}\left(n_{1}\right)\left[H_{L O S}^{p_{2}, q_{2}}\left(n_{1}\right)\right]^{*}\right\}
\end{aligned}
$$

Assuming uniform antenna arrays and uncorrelated scattering, i.e., $E\left\{a_{l}^{p_{1}, q_{1}}\left(a_{k}^{p_{2}, q_{2}}\right)^{*}\right\}=0$, when $l \neq k$, the above correlation becomes

$$
\begin{aligned}
& R_{L O S}\left(p_{1}, q_{1}, n_{1} ; p_{2}, q_{2}, n_{2}\right)= \\
& \frac{\left|a_{L O S}\right|^{2} f\left(\theta_{0}, \phi_{0}\right)+\sum_{l=1}^{L} \varepsilon_{l}^{2} \chi_{l}^{p_{1}, q_{1} ; p_{2}, q_{2}} \exp \left[-j 2 \pi \Delta f\left(n_{1}-n_{2}\right) \tau_{l}\right]}{\sum_{l=1}^{L} \varepsilon_{l}^{2} \chi_{l}^{p_{1}, q_{1} ; p_{2}, q_{2}}+\left|a_{L O S}\right|^{2} f\left(\theta_{0}, \phi_{0}\right)}
\end{aligned}
$$

where $f\left(\theta_{0}, \phi_{0}\right)=\exp \left[-j 2 \pi\left(p_{1}-p_{2}\right) d_{t} \sin \theta_{0}-j 2 \pi\left(q_{1}-q_{2}\right) d_{r} \sin \phi_{0}\right], d_{t}, d_{r}$ are the transmit and receive antenna spacing normalized to the wavelength, $\theta_{0}$ is the direction-ofdeparture (DOD), $\phi_{0}$ is the direction-of-arrival (DOA), and $\chi_{l}^{p_{1}, q_{1} ; p_{2}, q_{2}}$ is the spatial cor- 
relation coefficient, $\chi_{l}^{p_{1}, q_{1} ; p_{2}, q_{2}}=E\left\{a_{l}^{p_{1}, q_{1}}\left(a_{l}^{p_{2}, q_{2}}\right)^{*}\right\} / \varepsilon_{l}^{2}$.

When LOS is not available, the subcarrier correlation is obtained by letting $a_{L O S}=0$ in (5.5), that corresponds to

$$
\begin{aligned}
& R_{N L O S}\left(p_{1}, q_{1}, n_{1} ; p_{2}, q_{2}, n_{2}\right)= \\
& \frac{\sum_{l=1}^{L} \varepsilon_{l}^{2} \chi_{l}^{p_{1}, q_{1} ; p_{2}, q_{2}} \exp \left[-j 2 \pi \Delta f\left(n_{1}-n_{2}\right) \tau_{l}\right]}{\sum_{l=1}^{L} \varepsilon_{l}^{2} \chi_{l}^{p_{1}, q_{1} ; p_{2}, q_{2}}}
\end{aligned}
$$

\subsubsection{Definition of subcarrier correlation difference}

LOS subcarrier correlation difference (SCD) is defined as,

$$
\begin{aligned}
& S C D_{L O S}\left(p_{1}-p_{2}, q_{1}-q_{2}, n_{1}-n_{2}\right) \triangleq \\
& R_{L O S}\left(p_{1}, q_{1}, n_{1} ; p_{2}, q_{2}, n_{2}\right)-R_{L O S}\left(p_{1}, q_{1}, n_{1} ; p_{1}, q_{1}, n_{2}\right)
\end{aligned}
$$

NLOS subcarrier correlation difference is similarly defined and can be expanded as

$$
\begin{aligned}
& \operatorname{SCD}_{N L O S}\left(p_{1}-p_{2}, q_{1}-q_{2}, n_{1}-n_{2}\right) \triangleq \\
& R_{N L O S}\left(p_{1}, q_{1}, n_{1} ; p_{2}, q_{2}, n_{2}\right)-R_{N L O S}\left(p_{1}, q_{1}, n_{1} ; p_{1}, q_{1}, n_{2}\right) \\
& =\frac{\sum_{l=1}^{L} \varepsilon_{l}^{2} \chi_{l}^{p_{1}, q_{1} ; p_{2}, q_{2}} \exp \left[-j 2 \pi \Delta f\left(n_{1}-n_{2}\right) \tau_{l}\right]}{\sum_{l=1}^{L} \varepsilon_{l}^{2} \chi_{l}^{p_{1}, q_{1} ; p_{2}, q_{2}}} \\
& -\frac{\sum_{l=1}^{L} \varepsilon_{l}^{2} \exp \left[-j 2 \pi \Delta f\left(n_{1}-n_{2}\right) \tau_{l}\right]}{\sum_{l=1}^{L} \varepsilon_{l}^{2}}
\end{aligned}
$$




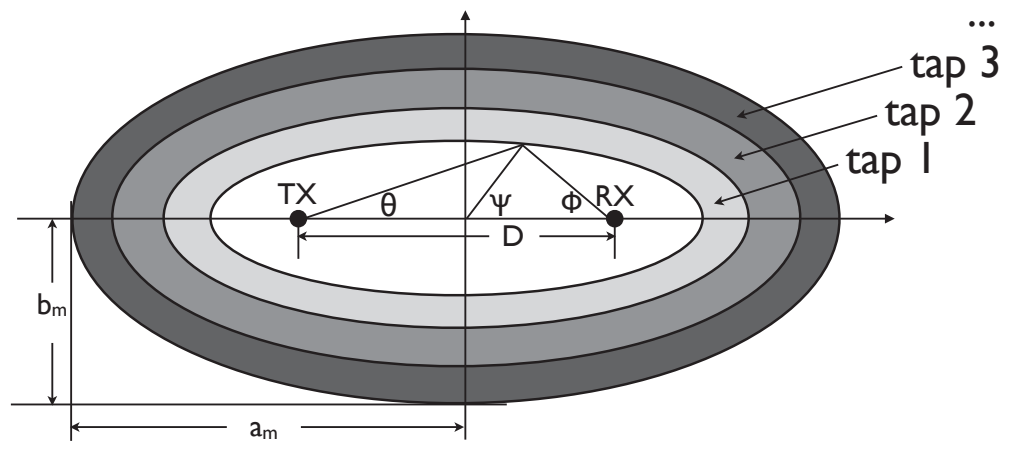

Figure 5.1: Geometry of scatterer distribution for mircocell environments

Now, we depict that in microcell environments $\chi_{l}^{p_{1}, q_{1} ; p_{2}, q_{2}}$ remains approximately unchanged across all multipath components, i.e.,

$$
\chi_{l}^{p_{1}, q_{1} ; p_{2}, q_{2}} \approx \chi^{p_{1}, q_{1} ; p_{2}, q_{2}}
$$

This renders $S C D_{N L O S}$ small (almost zero) compared to $S C D_{L O S}$ defined in (5.7). That is

$$
\operatorname{SCD}_{N L O S}\left(p_{1}-p_{2}, q_{1}-q_{2}, n_{1}-n_{2}\right) \approx 0
$$

Figure 5.1 depicts the geometry of a microcell environment where scatterers are distributed in the shaded area, the transmitter and the receiver are located at the foci of a set of ellipses, and the scatterers in each elliptical subregion (shown in a different shade) contribute to one of the multi-path components (taps). The spatial correlation $\chi_{l}^{p_{1}, q_{1} ; p_{2}, q_{2}}$ is determined by 
the power angular function of the $l^{\text {th }}$ multi-path component, i.e., $g_{l}(\psi)$, that is [77]

$$
\begin{aligned}
& \chi_{l}^{p_{1}, q_{1} ; p_{2}, q_{2}}= \\
& \int_{0}^{2 \pi} g_{l}(\psi) \exp \left(-j 2 \pi\left(p_{1}-p_{2}\right) d_{t} \sin \theta-j 2 \pi\left(q_{1}-q_{2}\right) d_{r} \sin \phi\right) d \psi
\end{aligned}
$$

where $\theta, \phi$ and $\psi$ are shown in Figure 5.1. $\psi$ is the angle of a scatterer with respect to the center of the ellipse. Note that $\theta$ and $\phi$ are related with $\psi$ by

$$
\begin{aligned}
& \sin \theta=\frac{R_{l} \sin \psi}{\sqrt{\left(R_{l} \sin \psi\right)^{2}+\left(D / 2+R_{l} \cos \psi\right)^{2}}} \\
& \sin \phi=\frac{R_{l} \sin \psi}{\sqrt{\left(R_{l} \sin \psi\right)^{2}+\left(D / 2-R_{l} \cos \psi\right)^{2}}}
\end{aligned}
$$

where $D$ is the distance between transceivers and

$$
R_{l}=\sqrt{1 /\left(\frac{\sin ^{2} \psi}{a_{l}^{2}}+\frac{\cos ^{2} \psi}{b_{l}^{2}}\right)}
$$

In (5.14), $a_{l}$ is the value of semi major axis, $b_{l}$ is the value of semi minor axis.

$g_{l}(\psi)$ in (5.11) is the power angular function which describes how the power is distributed with $\psi$ for the $l^{t h}$ multi-path component. Here, $g_{l}(\psi)=\frac{1}{2 \pi}, \quad 0 \leq \psi<2 \pi$. The multi path signals arrive with an absolute delay less than $\tau_{m}=5 \mu$ s and therefore the maximum value of semi major axis is $a_{m}=c \tau_{m} / 2=750 m$ where $c$ is the speed of light. Then $a_{l}$ and $b_{l}$ in 


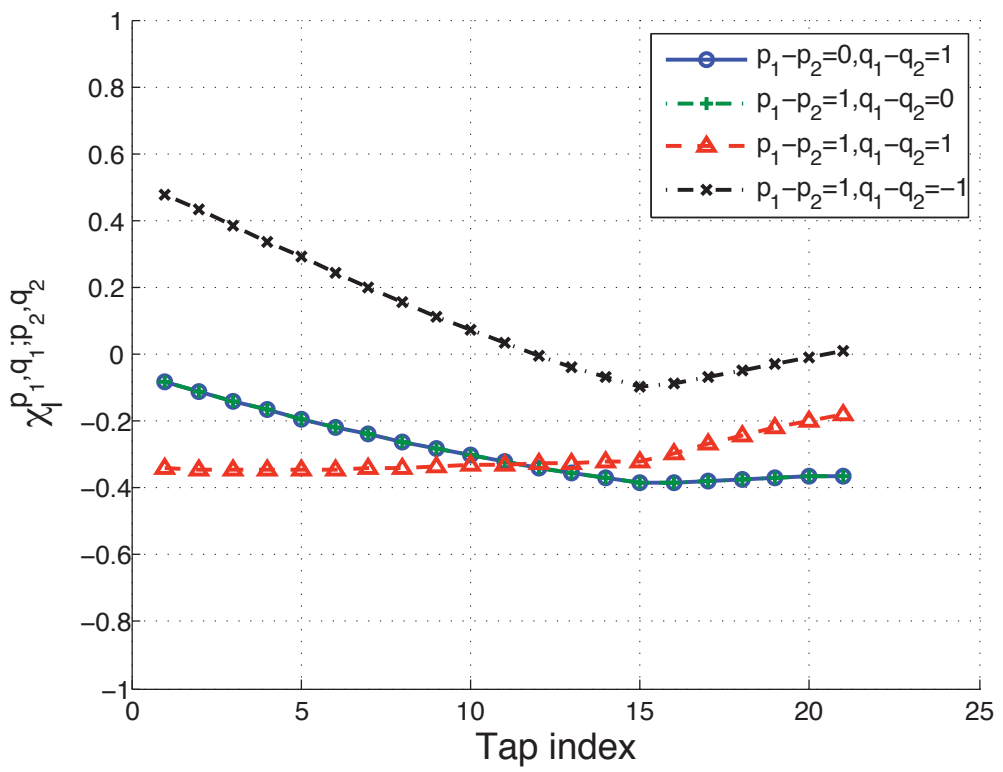

Figure 5.2: $\chi_{l}^{p_{1}, q_{1} ; p_{2}, q_{2}}$ in micro cell environment

(5.14) corresponds to

$$
\begin{aligned}
& a_{l}=a_{m}-(L-l) c \Delta_{\tau} / 2 \\
& b_{l}=\sqrt{a_{l}^{2}-(D / 2)^{2}}
\end{aligned}
$$

where $\Delta_{\tau}=50 \mathrm{~ns}$ for the system with $20 \mathrm{MHz}$ bandwidth. Plugging (5.12) (5.13) (5.15) (5.16) into (5.11), $\chi_{l}^{p_{1}, q_{1} ; p_{2}, q_{2}}$ is numerically computed and plotted in Figure 5.2 for different transmit and receive antenna combinations. It is noted that here $\chi_{l}^{p_{1}, q_{1} ; p_{2}, q_{2}}$ is a real number due to the symmetric scatterer distribution around the major axis. In Figure 5.2, it is observed that the variation of $\chi_{l}^{p_{1}, q_{1} ; p_{2}, q_{2}}$ over taps are small and the value of $\chi_{l}^{p_{1}, q_{1} ; p_{2}, q_{2}}$ does not cross 0 for most antenna combinations. Those observations support the approximation shown in (5.9). Note that the small variation of $\chi_{l}^{p_{1}, q_{1} ; p_{2}, q_{2}}$ is mainly determined by the fact that the DOA distributions of different multipath components are similar. This 
fact is also held for macrocell environments $[78,79]$ where the base station is usually elevated and free of local scatterer, and the mobile station is surrounded by a circle of local scatterers.

\subsubsection{Characteristics of SCD and SCD statistics across antenna pairs}

The absolute value of SCD can be used to distinguish LOS and NLOS. This is due to the fact that based on (5.7) and (5.10), the absolute value of SCD under LOS condition is greater than zero and the absolute value of SCD under NLOS condition is close to zero.

Let

$$
\begin{gathered}
\Gamma_{L O S}\left(n_{1}-n_{2}\right)=\left|S C D_{L O S}\left(p_{1}-p_{2}, q_{1}-q_{2}, n_{1}-n_{2}\right)\right| \\
\Gamma_{N L O S}\left(n_{1}-n_{2}\right)=\left|S C D_{N L O S}\left(p_{1}-p_{2}, q_{1}-q_{2}, n_{1}-n_{2}\right)\right|
\end{gathered}
$$

$\Gamma_{\operatorname{LOS}}\left(n_{1}-n_{2}\right)$ is a function of frequency separation $\left(n_{1}-n_{2}\right)$ and now it is decided which value of $\left(n_{1}-n_{2}\right)$ will be used. Observing (5.5) and (5.7), it is found that when $\left(n_{1}-\right.$ $\left.n_{2}\right)$ is small, both the first and the second correlation in (5.7) is close to 1. Accordingly, $\Gamma_{L O S}\left(n_{1}-n_{2}\right)$ would be close to zero and $\Gamma_{L O S}\left(n_{1}-n_{2}\right)$ and $\Gamma_{N L O S}\left(n_{1}-n_{2}\right)$ would be 
indistinguishable. Therefore, let $n_{1}-n_{2}=N / 2$, and $\Gamma_{L O S}(N / 2)$ is approximated by

$$
\begin{aligned}
& \Gamma_{L O S}(N / 2) \approx\left|\frac{\alpha}{\sum_{l=1}^{L} \varepsilon_{l}^{2} \chi_{l}^{p_{1}, q_{1} ; p_{2}, q_{2}}+\alpha}-\frac{\alpha}{\sum_{l=1}^{L} \varepsilon_{l}^{2}+\alpha}\right|
\end{aligned}
$$

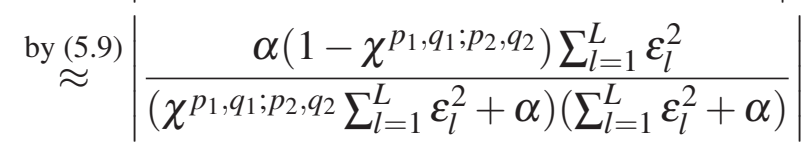

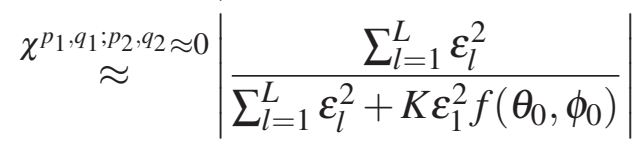

where $\alpha=\left|a_{L O S}\right|^{2} f\left(\theta_{0}, \phi_{0}\right)$. In (5.19), when $\chi^{p_{1}, q_{1} ; p_{2}, q_{2}} \approx 0, \Gamma_{L O S}(N / 2)$ decreases with Rician $K$ factor. Accordingly, $\Gamma_{L O S}(N / 2)$ cannot be distinguished from $\Gamma_{N L O S}(N / 2)$ when Rician $K$ factor is large.

The mean and variance of SCD of different transmit-receive antenna combinations can also be used to identify NLOS conditions. Note that (5.10) holds for any transmit and receive antenna combinations. Accordingly, the variation of $S C D_{N L O S}\left(p_{1}-p_{2}, q_{1}-q_{2}, N / 2\right)$ over different antenna combinations is minimal. For LOS conditions, let $\mu_{\mathrm{LOS}}$ denote the mean of absolute SCD values over antenna combinations and $\mu_{\mathrm{LOS}}^{\mathrm{abs}}$ denote the absolute mean value of SCD, i.e.,

$$
\begin{aligned}
& \mu_{\mathrm{LOS}}=\frac{1}{M} \sum_{p_{1}-p_{2}, q_{1}-q_{2}}\left|S C D_{\operatorname{LOS}}\left(p_{1}-p_{2}, q_{1}-q_{2}, N / 2\right)\right| \\
& \mu_{\mathrm{LOS}}^{\mathrm{abs}}=\frac{1}{M}\left|\sum_{p_{1}-p_{2}, q_{1}-q_{2}} S C D_{\operatorname{LOS}}\left(p_{1}-p_{2}, q_{1}-q_{2}, N / 2\right)\right|
\end{aligned}
$$

where $M$ is the number of antenna combinations. The standard deviation of absolute SCD 
values is denoted by $\sigma_{\mathrm{LOS}}$ and the absolute value of standard deviation of SCD is denoted by $\sigma_{\mathrm{LOS}}^{\mathrm{abs}}$. Similarly, $\mu_{\mathrm{NLOS}}, \mu_{\mathrm{NLOS}}^{\mathrm{abs}}, \sigma_{\mathrm{NLOS}}$ and $\sigma_{\mathrm{NLOS}}^{\mathrm{abs}}$ are defined for NLOS SCD values.

Based on (5.10) and (5.7), it is expected that

$$
\begin{aligned}
& \Gamma_{N L O S}(N / 2)<\Gamma_{L O S}(N / 2) \\
& \mu_{\mathrm{NLOS}}<\mu_{\mathrm{LOS}}, \quad \mu_{\mathrm{NLOS}}^{\mathrm{abs}}<\mu_{\mathrm{LOS}}^{\mathrm{abs}} \\
& \sigma_{\mathrm{NLOS}}<\sigma_{\mathrm{NLOS}}, \quad \sigma_{\mathrm{NLOS}}^{\mathrm{abs}}<\sigma_{\mathrm{LOS}}^{\mathrm{abs}}
\end{aligned}
$$

In Section 5.3, the performance of using $\Gamma_{N L O S}(N / 2), \mu_{\mathrm{NLOS}}$ and $\sigma_{\mathrm{NLOS}}$ in identifying NLOS conditions will be evaluated and compared.

\subsection{Numerical simulations and discussions}

Simulations are conducted to plot distributions of the absolute values, the mean values, the std values of SCD shown in (5.22)-(5.24). The performance of those measures in identifying NLOS scenarios are evaluated. Simulation parameters are selected consistent to 802.11n wireless LAN (WLAN) channel models [75]. Here, the bandwidth is $W=20$ $\mathrm{MHz}$, the number of subcarrier is $N=64, \Delta f=W / N=312.5 \mathrm{kHz}, d_{t}=d_{r}=\lambda / 2$. WLAN Channel Model F which is suitable for indoor or outdoor large open space is used here to characterize the power delay profile [75]. The delay spread $\tau_{r m s}=150 \mathrm{~ns}$, the maximum TOA $\tau_{\max }=1000 \mathrm{~ns}$, and the tap power is assumed to decay exponentially, i.e., 


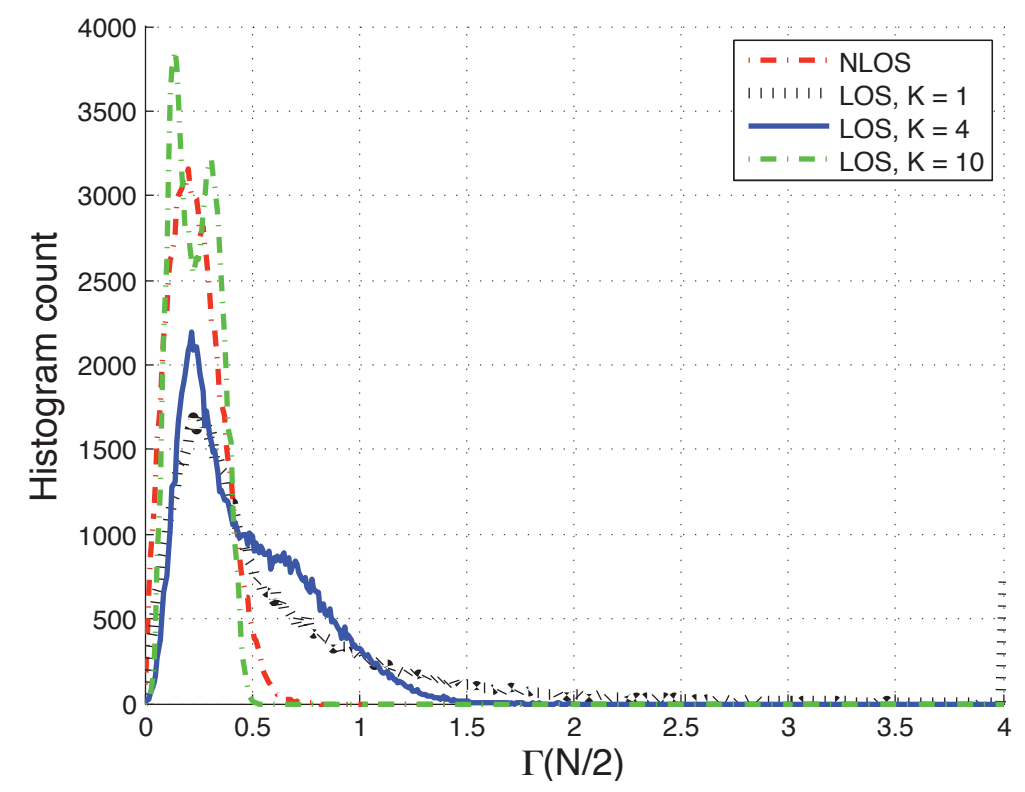

Figure 5.3: The distributions of $\Gamma(N / 2)$ under LOS and NLOS scenarios when $p 1-p 2=-1, q_{1}-q_{2}=-1$, Rician $K=1,4,10$.

$\varepsilon_{l}^{2}=\exp \left(-\tau_{l} / \tau_{r m s}\right)$

The spatial correlation $\chi_{l}^{p_{1}, q_{1} ; p_{2}, q_{2}}$ is assumed to be uniformly distributed in a hollow disk with radius from 0.1 to 0.9 on the complex plane, i.e., $\left|\chi_{l}^{p_{1}, q_{1} ; p_{2}, q_{2}}\right| \sim U[0.1,0.9]$, angle $\left(\chi_{l}^{p_{1}, q_{1} ; p_{2}, q_{2}}\right) \sim U[0,2 \pi]$, and the magnitude and the angle are independent. In order to maintain the similarity of $\chi_{l}^{p_{1}, q_{1} ; p_{2}, q_{2}}$ over taps, the spatial correlation values of all taps are restricted in either of the four quadrants. The DOD $\theta_{0}$ and the DOA $\phi_{0}$ are assumed to be independent and uniformly distributed in $[0,2 \pi]$, i.e., $\theta_{0}, \phi_{0} \sim U[0,2 \pi]$. 


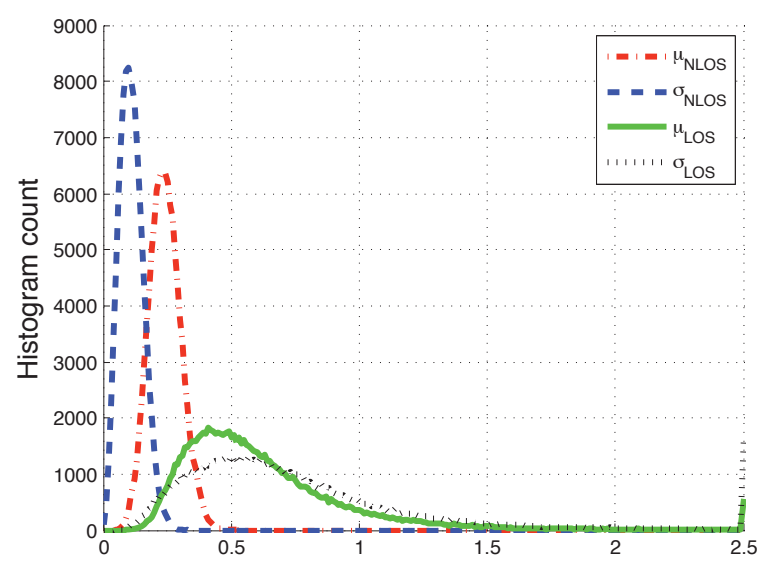

(a)

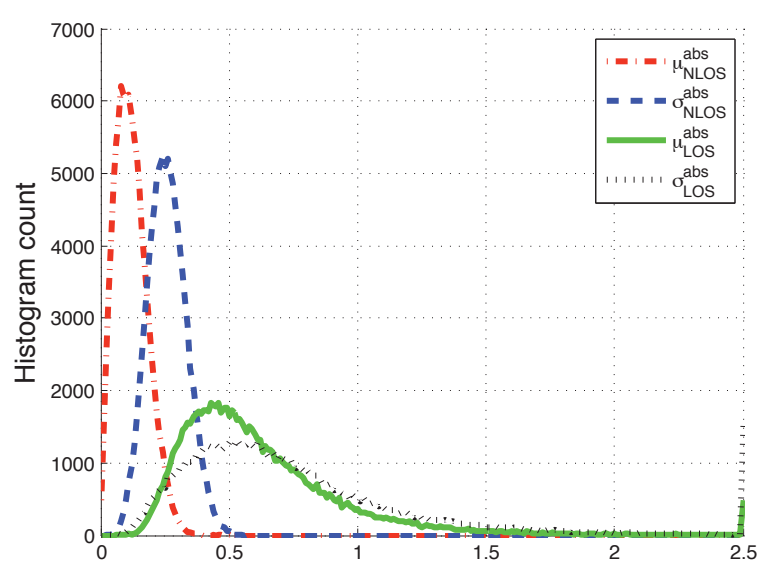

(b)

Figure 5.4: The distributions of (a) $\mu$ and $\sigma$ (b) $\mu^{\text {abs }}$ and $\sigma^{\text {abs }}$ when Rician $K=1$.

\subsubsection{Absolute value of SCD}

The distributions of $\Gamma(N / 2)$ in (5.22) under LOS and NLOS scenarios for different values of Rician $K$ factor are shown in Figure 5.3. It is observed that SCD values for LOS and NLOS are almost indistinguishable due to the large overlap of the distributions. In particular, when $K=10$, the distributions of $\Gamma_{N L O S}(N / 2)$ and $\Gamma_{L O S}(N / 2)$ are almost identical. Therefore, the identification performance of $\Gamma(N / 2)$ is poor.

\subsubsection{Mean and variance of SCD}

A $2 \times 2$ MIMO system is considered. The transmit antenna difference $\left(p_{1}-p_{2}\right)$ and the receive antenna difference $\left(q_{1}-q_{2}\right)$ take on values from $\{0,1,-1\}$. Therefore, 4 distinct $\mathrm{SCD}$ values can be computed using the antenna combination $\left(p_{1}-p_{2}, q_{1}-q_{2}\right)$ from the set $\{(0,1)(1,0)(1,1)(1,-1)\}$. 
The distributions of mean values and std values of SCD are shown in Figure 5.4. Small overlaps of mean and std distributions for LOS and NLOS are seen which indicates a good NLOS detection performance. Bayesian hypothesis testing [70] is applied to perform the NLOS detection and the decision rules are as follows,

\section{Decide NLOS if}

$\mu<\mu_{\text {th }}$ or $\mu^{\mathrm{abs}}<\mu_{\mathrm{th}}^{\mathrm{abs}}$ or $\sigma<\sigma_{\mathrm{th}}$ or $\sigma^{\mathrm{abs}}<\sigma_{\mathrm{th}}^{\mathrm{abs}}$

Decide LOS if

$$
\mu>\mu_{\mathrm{th}} \text { or } \mu^{\mathrm{abs}}>\mu_{\mathrm{th}}^{\mathrm{abs}} \text { or } \sigma>\sigma_{\mathrm{th}} \text { or } \sigma^{\mathrm{abs}}>\sigma_{\mathrm{th}}^{\mathrm{abs}}
$$

The detection performance is characterized by the false alarm rate $P_{F}$ and the detection rate $P_{D}$

$$
\begin{aligned}
& P_{F}=P(\operatorname{NLOS} \mid \mathrm{LOS})=\int_{0}^{\eta} f(m \mid \operatorname{LOS}) d m \\
& P_{D}=P(\operatorname{NLOS} \mid \mathrm{NLOS})=\int_{\eta}^{\infty} f(m \mid \mathrm{NLOS}) d m
\end{aligned}
$$

where $\eta$ represent the decision threshold, $f(m \mid$ LOS $)$ is the metric pdf under LOS and $f(m \mid$ NLOS $)$ is the metric pdf under NLOS. Now, the decision threshold, false alarm rate $P_{F}$ and detection rate $P_{D}$ are computed. Assuming the probability of LOS and NLOS appearances are equal, the decision threshold for each mean or std metric would be the intersection of corresponding pdf curves for LOS and NLOS conditions. Accordingly, 
Table 5.1: Comparison of decision threshold, $P_{F}$ and $P_{D}$ for various metrics when Rician $K=1$

\begin{tabular}{|c|c|c|c|c|}
\hline & $\mu$ & $\sigma$ & $\mu^{\mathrm{abs}}$ & $\sigma^{\mathrm{abs}}$ \\
\hline Threshold & $\mu_{\mathrm{th}}=0.34$ & $\sigma_{\mathrm{th}}=0.22$ & $\mu_{\mathrm{th}}^{\mathrm{abs}}=0.26$ & $\sigma_{\mathrm{th}}^{\mathrm{abs}}=0.39$ \\
\hline$P_{F}$ & $15.68 \%$ & $3.96 \%$ & $4.31 \%$ & $19.50 \%$ \\
\hline$P_{D}$ & $94.86 \%$ & $98.77 \%$ & $97.26 \%$ & $95.92 \%$ \\
\hline
\end{tabular}

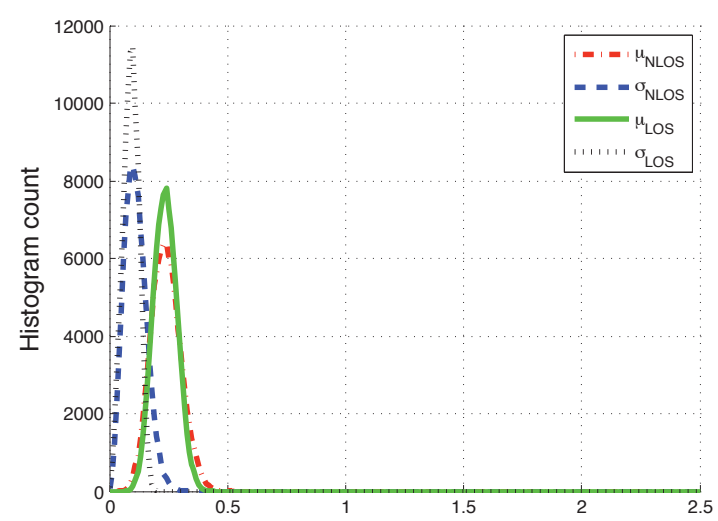

(a)

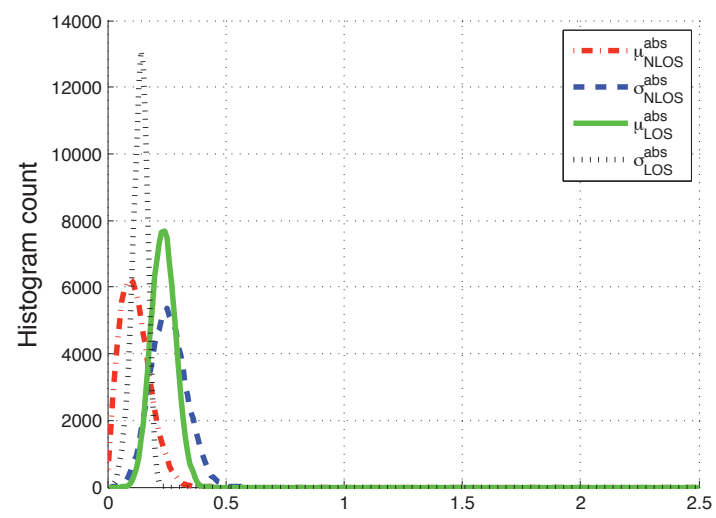

(b)

Figure 5.5: The distributions of (a) $\mu$ and $\sigma$ (b) $\mu^{\text {abs }}$ and $\sigma^{\text {abs }}$ when Rician $K=10$.

decision threshold, $P_{F}$ and $P_{D}$ are summarized in Table 5.1. It is shown in the table that $\sigma$ and $\mu^{\text {abs }}$ offers the best detection performance, since the detection using those two metrics have a low false alarm rate and a high detection rate.

Next the impact of Rician $K$ on the performance of mean and std values is discussed. Following the same argument below (5.19), as Rician $K$ increases, $\Gamma(N / 2)$ decreases and both the mean and std values of SCD under LOS will be pushed near to those under NLOS. This analysis is confirmed in Figure 5.5 where mean and std distributions are plotted for Rician $K=10$. (Note that in elliptical or circular scattering model, the Rician $K$ can hardly exceed 10 due to rich scattering.) It is observed that the distributions of $\mu_{\mathrm{LOS}}$ and $\mu_{\mathrm{NLOS}}$ are almost identical, the distributions of $\sigma_{\mathrm{LOS}}$ and $\sigma_{\mathrm{NLOS}}$ are almost identical, and with a large 
probability $\sigma_{\mathrm{LOS}}^{\mathrm{abs}}<\sigma_{\mathrm{NLOS}}^{\mathrm{abs}}$. Only $\mu^{\text {abs }}$ can still be used to identify NLOS for large Rician $K$, and the threshold, probability of detection and probability of false alarm are $\mu_{t h}^{a b s}=0.17$, $P_{F}=11.43 \%, P_{D}=82.23 \%$. Therefore, the performance of large Rician $K$ is worse than that of small Rician $K$.

\subsection{Conclusion}

This chapter proposes to use space-frequency channel correlation based metrics and spacetime-frequency channel correlation based metrics for NLOS identification in MIMO-OFDM systems.

It is observed that in elliptical scattering microcell environments, SCD in NLOS scenarios is approximately 0 for any transmit and receive antenna combinations, but SCD in LOS scenarios would change with selected antenna combination. Therefore, the absolute value, the mean value and the standard deviation of SCD of NLOS are probably smaller than those of LOS. Simulations using WLAN $2 \times 2$ MIMO channel model shows that the technique using the mean and standard deviation has a higher identification performance than the technique using the absolute value of SCD. Among the four proposed mean and standard deviation metrics, identification using $\sigma$ and $\mu^{\text {abs }}$ offers the best performance, maintaining a high detection rate (around 98\%) with a low false alarm rate (around 4\%) for small values of Rician $K$ (e.g. in the order of one). For large values of Rician $K$, the detection perfor-

mance is poor and only $\mu^{\text {abs }}$ can offers a decent performance. These results confirm that 
the proposed technique is a good candidate for NLOS identification that can be employed in MIMO-OFDM systems. 


\section{Chapter 6}

\section{NLOS Identification in Time-Varying, Frequency Selective and Space Selective radio channels}

This chapter proposes new measures for non-line-of-sight (NLOS) identification in wireless localization systems. The measures are defined based on the space-time-frequency channel correlation features of multi-input multi-output (MIMO) orthogonal frequency division multiplexing (OFDM) systems. Here, space-time channel correlation refers to the correlation across antenna elements and time, and frequency channel correlation refers to the correlation across subcarriers. The probability-of-detection performance of these NLOS identification methods is investigated. The results represent a high identification performance when the LOS and NLOS powers are in the same order of magnitude. 


\subsection{Introduction}

In this chapter, we propose a new NLOS identification technique exploiting space-timefrequency channel correlation of MIMO-OFDM systems. The channel correlation of MIMOOFDM systems can be measured across antenna (space), across time and subcarrier (frequency). The metrics proposed to perform NLOS identification include: (1) the absolute value of space-time channel correlation with subcarrier separation and (2) the absolute value of space-time channel correlation with zero subcarrier separation and (3) a function of the first and the second metrics. The identification process is based on the idea that the correlation of NLOS components would approach zero as the space and time separation increase while the absolute value of the LOS component correlation is constant with any space and time separation. The capability of the proposed measures are analyzed theoretically and via simulations.

The remainder of this chapter is organized as follows. In Section 6.2, we introduce the space-time-frequency channel correlation model of MIMO-OFDM systems. In Section 6.3, the metrics used in NLOS identification are defined. In Section 6.4, we present the performance of NLOS identification using the proposed metrics. Section 6.5 concludes this chapter. 


\subsection{Space-time-frequency channel model of MIMO-OFDM systems}

We consider a wideband MIMO channel with $M_{t}$ and $M_{r}$ transmit and receive antenna elements, respectively. The time-varying, time-dispersion channel impulse response (CIR) from the $p^{\text {th }}\left(p=1,2, \ldots M_{t}\right)$ transmit antenna to the $q^{\text {th }}\left(q=1,2, \ldots M_{r}\right)$ receive antenna is represented by a tapped-delay-line model that corresponds to

$$
h^{p, q}(t, \tau)=\sum_{l=1}^{L} a_{l}^{p, q}(t) \delta\left(\tau-\tau_{l}\right)
$$

where $a_{l}^{p, q}(t)$ is the amplitude coefficient of the $l^{t h}$ multi-path component, and $\tau_{l}$ is the associated TOA. When LOS is not available, let $\varepsilon_{l}^{2}$ be the power of the $l^{\text {th }}$ tap for any transmit-receive antenna combination, i.e., $\varepsilon_{l}^{2}=E\left(\left|a_{l}^{p, q}(t)\right|^{2}\right)$. When LOS component is available, the first tap is the sum of a specular component and a diffuse component, i.e., $a_{1}^{p, q}(t)=a_{s}^{p, q}(t)+a_{d}^{p, q}(t)$. Let $\left|a_{s}^{p, q}(t)\right|^{2}=\sigma_{s}^{2}$ and $E\left(\left|a_{d}^{p, q}(t)\right|^{2}\right)=\varepsilon_{1}^{2}$. Then the Rician $K$-factor of the first path is defined as

$$
K \triangleq \frac{\left|a_{s}^{p, q}(t)\right|^{2}}{E\left(\left|a_{d}^{p, q}(t)\right|^{2}\right)}=\frac{\sigma_{s}^{2}}{\varepsilon_{1}^{2}}
$$


Assuming the receiver is moving, the specular component $a_{s}^{p, q}(t)$ corresponds to

$$
a_{s}^{p, q}(t)=A \exp \left(-j 2 \pi(p-1) d_{t} \sin \theta_{0}-j 2 \pi(q-1) d_{r} \sin \phi_{0}\right) \exp \left(j 2 \pi f_{D} \sin \left(\phi_{0}-\phi_{\alpha}\right) t\right)
$$

where $A$ is a constant, $\theta_{0}$ is the direction-of-departure (DOD) of LOS component, $\phi_{0}$ is the DOA of LOS component, $f_{D}$ is the maximum doppler frequency in radian, and $\phi_{\alpha}$ is the direction of movement.

Next, we compute the channel response in the frequency domain. Let the signal bandwidth be $W$ and the total number of subcarriers is $N$, the frequency spacing in OFDM is defined as $\Delta f \triangleq W / N$. Applying Fourier transform to (6.1), the channel frequency response of the $n^{\text {th }}$ subcarrier is obtained as

$$
H^{p, q}(n, t)=\sum_{l=1}^{L} a_{l}^{p, q}(t) \exp \left(-j 2 \pi n \Delta f \tau_{l}\right)
$$

where $n=0,1, \ldots N-1$.

Using (6.4), the space-time-frequency correlation of $H^{p_{1}, q_{1}}\left(n_{1}, t\right)$ and $H^{p_{2}, q_{2}}\left(n_{2}, t+\Delta t\right)$ is 
expressed as

$$
\begin{aligned}
& R\left(p_{1}-p_{2}, q_{1}-q_{2}, n_{1}-n_{2}, \Delta t\right) \\
& \triangleq E\left[H^{p_{1}, q_{1}}\left(n_{1}, t\right) H^{p_{2}, q_{2}}\left(n_{2}, t+\Delta t\right)^{*}\right] \\
& =\sum_{l=1}^{L} E\left[a_{l}^{p_{1}, q_{1}}(t) a_{l}^{p_{2}, q_{2}}(t+\Delta t)^{*}\right] \exp \left(-j 2 \pi \Delta f\left(n_{1}-n_{2}\right) \tau_{l}\right)
\end{aligned}
$$

which is assumed to be wide-sense stationary with time [64]. Since (6.5) is a function of $\left(n_{1}-n_{2}\right)$, it is stationary with the subcarrier separation. In addition, the space-timefrequency correlation is also stationary with the space separation, because as shown in [77] the term $E\left[a_{l}^{p_{1}, q_{1}}(t) a_{l}^{p_{2}, q_{2}}(t+\Delta t)^{*}\right]$ in (6.5) is a function of $\left(p_{1}-p_{2}\right)$ and $\left(q_{1}-q_{2}\right)$. Therefore, the space-time-frequency correlation (6.5) is stationary with the space separation, subcarrier separation and time separation. Then the correlation coefficients corresponds to

$$
\begin{aligned}
& \rho\left(p_{1}-p_{2}, q_{1}-q_{2}, n_{1}-n_{2}, \Delta t\right) \\
& \triangleq \frac{R\left(p_{1}-p_{2}, q_{1}-q_{2}, n_{1}-n_{2}, \Delta t\right)}{R(0,0,0,0)} \\
& =\frac{\sum_{l=1}^{L} R_{a_{l}}\left(p_{1}-p_{2}, q_{1}-q_{2}, \Delta t\right) \exp \left(-j 2 \pi \Delta f\left(n_{1}-n_{2}\right) \tau_{l}\right)}{\sum_{l=1}^{L} R_{a_{l}}(0,0,0)}
\end{aligned}
$$

where $R_{a_{l}}\left(p_{1}-p_{2}, q_{1}-q_{2}, \Delta t\right)=E\left[a_{l}^{p_{1}, q_{1}}(t) a_{l}^{p_{2}, q_{2}}(t+\Delta t)^{*}\right]$.

When the LOS component is available, $R_{a_{1}}\left(p_{1}-p_{2}, q_{1}-q_{2}, \Delta t\right)=R_{a_{s}}\left(p_{1}-p_{2}, q_{1}-q_{2}, \Delta t\right)+$ 
$R_{a_{d}}\left(p_{1}-p_{2}, q_{1}-q_{2}, \Delta t\right)$ and according to (6.3),

$$
\begin{aligned}
& R_{a_{s}}\left(p_{1}-p_{2}, q_{1}-q_{2}, \Delta t\right) \\
& =\sigma_{s}^{2} \exp \left(-j 2 \pi\left(p_{1}-p_{2}\right) d_{t} \sin \theta_{0}-j 2 \pi\left(q_{1}-q_{2}\right) d_{r} \sin \phi_{0}\right) \exp \left(j 2 \pi f_{D} \sin \left(\phi_{0}-\phi_{\alpha}\right) \Delta t\right)
\end{aligned}
$$

where $\sigma_{s}^{2}$ is the power of the LOS component.

\subsection{Proposed measures: $\Lambda(N / 2), \Lambda(0)$ and $\Omega$}

The proposed measures are defined as,

$$
\begin{aligned}
\Lambda(N / 2) & =\left|\rho\left(p_{1}-p_{2}, q_{1}-q_{2}, N / 2, \Delta t\right)\right| \\
& =\frac{\left|\sum_{l=2 k-1} R_{a_{l}}\left(p_{1}-p_{2}, q_{1}-q_{2}, \Delta t\right)-\sum_{l=2 k} R_{a_{l}}\left(p_{1}-p_{2}, q_{1}-q_{2}, \Delta t\right)\right|}{\sum_{l=1}^{L} R_{a_{l}}(0,0,0)}
\end{aligned}
$$

and,

$$
\begin{aligned}
\Lambda(0) & =\left|\rho\left(p_{1}-p_{2}, q_{1}-q_{2}, 0, \Delta t\right)\right| \\
& =\frac{\left|\sum_{l=2 k-1} R_{a_{l}}\left(p_{1}-p_{2}, q_{1}-q_{2}, \Delta t\right)+\sum_{l=2 k} R_{a_{l}}\left(p_{1}-p_{2}, q_{1}-q_{2}, \Delta t\right)\right|}{\sum_{l=1}^{L} R_{a_{l}}(0,0,0)}
\end{aligned}
$$


Now, we define:

$$
\begin{aligned}
\Omega & =\left|\frac{\Lambda(N / 2)+\Lambda(0)}{2}\right|^{2}-\left|\frac{\Lambda(N / 2)-\Lambda(0)}{2}\right|^{2} \\
& =\frac{\left|\sum_{l=2 k-1} R_{a_{l}}\left(p_{1}-p_{2}, q_{1}-q_{2}, \Delta t\right)\right|^{2}-\left|\sum_{l=2 k} R_{a_{l}}\left(p_{1}-p_{2}, q_{1}-q_{2}, \Delta t\right)\right|^{2}}{\left(\sum_{l=1}^{L} R_{a_{l}}(0,0,0)\right)^{2}}
\end{aligned}
$$

Considering $\left(n_{1}-n_{2}\right)=N / 2$, the second equality in (6.8) holds because the exponential term in the numerator of (6.6), $\exp \left(-j 2 \pi \Delta f\left(n_{1}-n_{2}\right) \tau_{l}\right)=\exp (-j \pi(l-1))=(-1)^{l-1}$ when $\Delta f=W / N$ and $\tau_{l}=(l-1) / W$, where $W$ is the bandwidth. Considering $\left(n_{1}-n_{2}\right)=0$, the second equality in (6.9) holds because the exponential term in the numerator of (6.6), $\exp \left(-j 2 \pi \Delta f\left(n_{1}-n_{2}\right) \tau_{l}\right)=1$

The correlation coefficient of two signals at a space separation greater than the coherence distance or a time separation greater than the coherence time would be small. In general, when the space separation or the time separation is sufficiently large, the corresponding correlation coefficient would be zero, i.e.,

$$
\lim _{\Delta t \rightarrow \infty \text { or }\left(p_{1}-p_{2}\right) \rightarrow \infty \text { or }\left(q_{1}-q_{2}\right) \rightarrow \infty} \Lambda_{N L O S}(N / 2)=0
$$

In NLOS cases, coherence time or distance is expected to be significantly small. Thus, $R_{a_{l}}\left(p_{1}-p_{2}, q_{1}-q_{2}, \Delta t\right)$ is expected to be small for typical separation of $p_{1}-p_{2} \geq 1$ (or $\left.q_{1}-q_{2} \geq 1\right)$, namely one wavelength separation. 
In LOS cases, the correlation of LOS component given in (6.7) has an absolute value of $\sigma_{s}^{2}$ which does not change with the space and time separation. Therefore,

$$
\lim _{\Delta t \rightarrow \infty \text { or }\left(p_{1}-p_{2}\right) \rightarrow \infty \text { or }\left(q_{1}-q_{2}\right) \rightarrow \infty} \Lambda_{L O S}(N / 2)=\frac{\sigma_{s}^{2}}{\sum_{l=1}^{L} R_{a_{l}}(0,0,0)}
$$

Based on the above observations, the idea behind using $\Lambda(N / 2)$ in NLOS identification is that in NLOS cases the numerator of those measures would be close to zero and in LOS cases the numerator of those measures would be around $\sigma_{s}^{2}$. Therefore, we expect that at a certain spacing and time separations,

$$
\Lambda_{L O S}(N / 2)>\Lambda_{N L O S}(N / 2)
$$

Following the same arguments, we also expect that at a certain spacing separation and time separation,

$$
\Lambda_{L O S}(0)>\Lambda_{N L O S}(0)
$$

and,

$$
\Omega_{L O S}>\Omega_{N L O S}
$$


In this technique, a high value of correlation $R_{a_{l}}\left(p_{1}-p_{2}, q_{1}-q_{2}, \Delta t\right)$ in NLOS case would be mistakenly identified as LOS case. Due to the combination of space separation and time separation, $R_{a_{l}}\left(p_{1}-p_{2}, q_{1}-q_{2}, \Delta t\right)$ would probably has a lower value than the correlation with space separation or time separation alone. Kronecker channel model [50] is considered here to model the correlation $R_{a_{l}}\left(p_{1}-p_{2}, q_{1}-q_{2}, \Delta t\right)$. Kronecker channel model has been used in wireless LAN 802.11n for MIMO system performance analysis [75].

It has been shown that considering Kronecker model, the space-time correlation can be represented by $[60,54]$

$$
\begin{aligned}
R_{a_{l}}\left(p_{1}-p_{2}, q_{1}-q_{2}, \Delta t\right) & =\varepsilon_{l}^{2} \rho_{l}\left(p_{1}-p_{2}, q_{1}-q_{2}, \Delta t\right) \\
& =\varepsilon_{l}^{2} \rho_{l}\left(p_{1}-p_{2}\right) \rho_{l}\left(q_{1}-q_{2}, \Delta t\right)
\end{aligned}
$$

where the correlation coefficients is the product of transmit space and receive space-time

correlation coefficients. In this case, the absolute value of the product of two correlation coefficients would be more likely to be smaller than the absolute value of single correlation coefficient, with a radius that varies from 0 to 1 on the complex plane.

\subsection{Numerical simulations and discussions}

Simulations are conducted to plot distributions of $\Lambda(N / 2), \Lambda(0)$ and $\Omega$ shown in (6.8)(6.10). The performance of those measures in identifying NLOS scenarios are evaluated. 


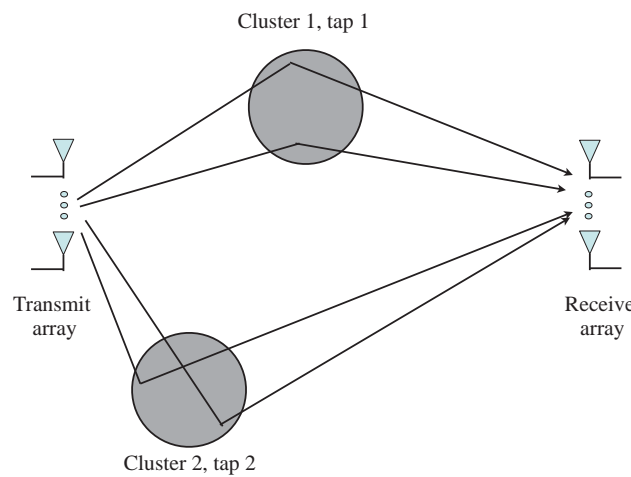

(a)

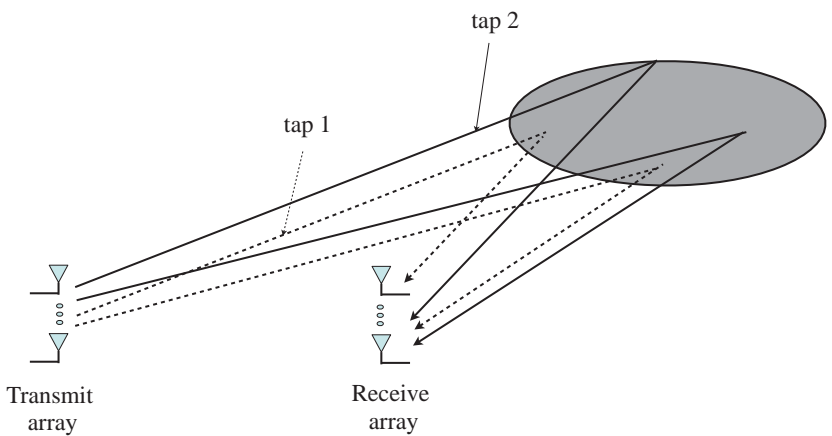

(b)

Figure 6.1: (a) Cluster scattering model A: $R_{a_{l}}$ is independent of $R_{a_{m}}, l \neq m$. (b) Cluster scattering model $\mathrm{B}, R_{a_{l}} \approx R_{a_{m}}, l \neq m$.

Simulation parameters are selected consistent to $802.11 \mathrm{n}$ wireless LAN (WLAN) channel models [75]. Here, the bandwidth is $W=20 \mathrm{MHz}$, the number of subcarrier is $N=64$, $\Delta f=W / N=312.5 \mathrm{kHz}, d_{t}=d_{r}=\lambda / 2$. WLAN Channel Model F which is suitable for indoor or outdoor large open space is used here to characterize the power delay profile [75]. The delay spread $\tau_{r m s}=150 \mathrm{~ns}$, the maximum TOA $\tau_{\max }=1000 \mathrm{~ns}$, and the tap power is assumed to decay exponentially, i.e., $\varepsilon_{l}^{2}=\exp \left(-\tau_{l} / \tau_{r m s}\right)$.

Here, the correlation coefficients $\rho_{l}\left(p_{1}-p_{2}\right)$ and $\rho_{l}\left(q_{1}-q_{2}, \Delta t\right)$ in (6.16) are assummed to be independent and uniformly distributed in the circle with radius from 0 to 1 on the complex plane.

In order to model the relationship of the correlation coefficients of different taps, such as $R_{a_{l}}\left(p_{1}-p_{2}, q_{1}-q_{2}, \Delta t\right)$ of tap $l$ and $R_{a_{m}}\left(p_{1}-p_{2}, q_{1}-q_{2}, \Delta t\right)$ of tap $m$, cluster scattering model A and B are considered. In cluster scattering model A shown in Figure 6.1(a), the location and the size of the cluster corresponding to one tap is independent of those of the 
cluster corresponding to another tap. In this case, correlation coefficients of different taps are assumed to be independent. This is due to the fact that the correlation coefficient of one tap is determined by the location and the size of the cluster of scatterers corresponding to that tap and independent clusters would lead to independent tap correlation. In cluster scattering model B shown in Figure 6.1(b), neighboring taps are formed by the same cluster and this creates some degrees of correlation between the correlation coefficients of neighboring taps [80], i.e., $R_{a_{l}} \approx R_{a_{m}}, l \neq m$. In the following simulation, similar neighboring taps correlation is realized by modeling the neighboring taps correlation coefficients to lie in the same quadrant of the complex plane.

The performance of $\Lambda(0)$ and $\Omega$ would not differ considerably between the two correlation coefficients models: independent correlation coefficients across taps and correlated correlation coefficients of neighboring taps. However, the performance of $\Lambda(N / 2)$ is expected to be better in the latter model. The numerator of $\Lambda(N / 2)$ in (6.8) can be rearranged as the sum of neighboring correlation difference. The sum is suppressed in the latter model and as a result the distribution of $\Lambda_{N L O S}(N / 2)$ is pushed away from that of $\Lambda_{L O S}(N / 2)$.

First, simulation results for cluster scattering model A are presented. Let $p_{1}-p_{2}=1$ and $q_{1}-q_{2}=1$. The symmetry in the correlation coefficient distribution would lead to the same performance of $\Lambda(0)$ and $\Lambda(N / 2)$. Therefore, only the distributions of $\Lambda(N / 2)$ and $\Omega$ are depicted in Figure 6.2 for Rician $K=0.5$. The probability of false alarm $P_{F}$ and the probability of detection $P_{D}$ for Rician $K=0.5,1,1.5,3$ are listed in Table 6.1. The 


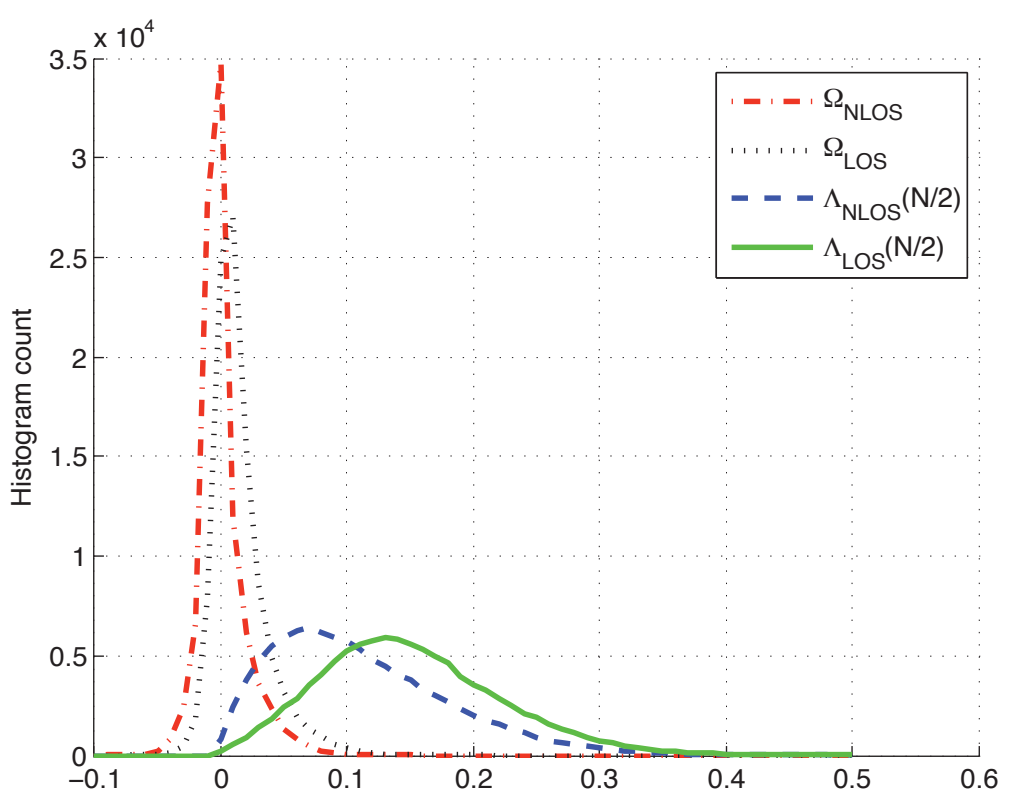

Figure 6.2: The distributions of $\Lambda(N / 2)$ and $\Omega$ when Rician $K=0.5$ for cluster scattering model A

Table 6.1: Comparison of $P_{F}$ and $P_{D}$ for $\Lambda(N / 2)$ and $\Omega$ when Rician $K=0.5,1$, $1.5,3$ for cluster scattering model A

\begin{tabular}{|c|c|c|c|c|}
\hline & $K=0.5$ & $K=1$ & $K=1.5$ & $K=3$ \\
\hline$\Lambda(N / 2), P_{F}$ & $27.63 \%$ & $15.26 \%$ & $7.29 \%$ & $0.48 \%$ \\
\hline$\Lambda(N / 2), P_{D}$ & $53.56 \%$ & $77.25 \%$ & $89.27 \%$ & $99.06 \%$ \\
\hline$\Omega, P_{F}$ & $35.74 \%$ & $9.20 \%$ & $4.46 \%$ & $0.13 \%$ \\
\hline$\Omega, P_{D}$ & $72.40 \%$ & $84.12 \%$ & $94.49 \%$ & $99.88 \%$ \\
\hline
\end{tabular}

threshold for computing $P_{F}$ and $P_{D}$ is the intersection of two corresponding distribution curves. From the table, it is observed that $\Omega$ has a better NLOS detection performance and the performance of both $\Lambda(N / 2)$ and $\Omega$ increases with Rician $K$. As predicted in (6.11), (6.12), larger $K$ leads to larger LOS measures. This creates larger gap between LOS measures and NLOS measures, which reduces the identification error and improves the performance. It can be concluded that either of the proposed measures would offer good performance when $K>1.5$. 


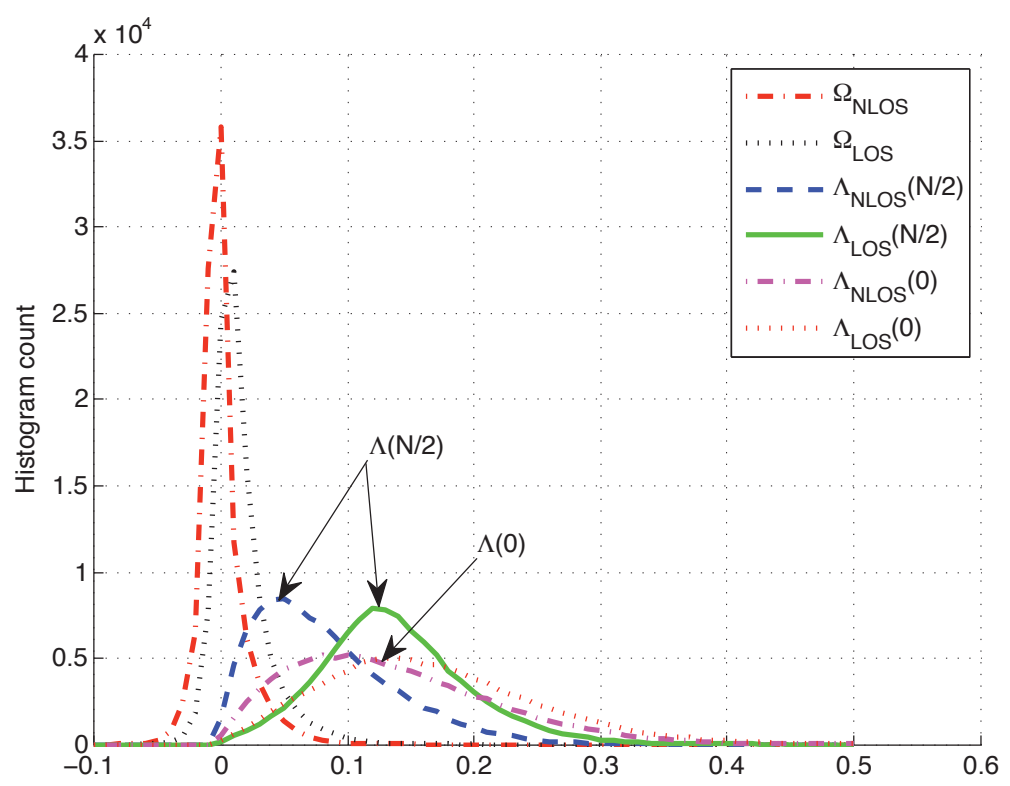

Figure 6.3: The distributions of $\Lambda(N / 2), \Lambda(0)$ and $\Omega$ when Rician $K=0.5$ for cluster scattering model B

Table 6.2: Comparison of $P_{F}$ and $P_{D}$ for $\Lambda(N / 2), \Lambda(0)$ and $\Omega$ when Rician $K=0.5$, $1,1.5,3$ for cluster scattering model $\mathrm{B}$

\begin{tabular}{|c|c|c|c|c|}
\hline & $K=0.5$ & $K=1$ & $K=1.5$ & $K=3$ \\
\hline$\Lambda(0), P_{F}$ & $34.49 \%$ & $19.71 \%$ & $12.11 \%$ & $1.64 \%$ \\
\hline$\Lambda(0), P_{D}$ & $51.44 \%$ & $69.15 \%$ & $83.66 \%$ & $97.72 \%$ \\
\hline$\Lambda(N / 2), P_{F}$ & $23.65 \%$ & $9.74 \%$ & $2.47 \%$ & $0.026 \%$ \\
\hline$\Lambda(N / 2), P_{D}$ & $66.10 \%$ & $89.66 \%$ & $95.98 \%$ & $99.94 \%$ \\
\hline$\Omega, P_{F}$ & $34.49 \%$ & $9.49 \%$ & $4.04 \%$ & $1.5 \%$ \\
\hline$\Omega, P_{D}$ & $73.20 \%$ & $85.29 \%$ & $94.89 \%$ & $99.88 \%$ \\
\hline
\end{tabular}

Next, simulation results for cluster scattering model B are presented. The distributions of $\Lambda(N / 2), \Lambda(0)$ and $\Omega$ for Rician $K=0.5$ are depicted in Figure 6.3.

The $P_{F}$ and $P_{D}$ for Rician $K=0.5,1,1.5,3$ are listed in Table 6.2. The threshold for computing $P_{F}$ and $P_{D}$ is the intersection of two corresponding distribution curves. From the table, it is observed that the performance of $\Lambda(N / 2)$ is better than that of $\Lambda(0)$, since $\Lambda(N / 2)$ takes the advantage of frequency selectivity and similar correlation of neighboring 
taps. The performance of $\Omega$ and $\Lambda(N / 2)$ are comparable. Again it is noted that the performance of all measures increases with Rician $K$ and either of the proposed measure would offer good performance when $K>1.5$.

\subsection{Conclusion}

This chapter proposes NLOS identification techniques for MIMO-OFDM systems by incorporating space-time-frequency channel correlation based metrics: $\Lambda(N / 2), \Lambda(0)$ and $\Omega$. It is observed that the detection performance of $\Omega$ beats that of $\Lambda(N / 2)$ and $\Lambda(0)$ when the space-time tap correlation is independent from tap to tap (cluster scattering model A); the detection performance of $\Omega$ and $\Lambda(N / 2)$ is comparable and is better than $\Lambda(0)$ as the space-time tap correlation of neighboring taps is similar (cluster scattering model B). For both cases, the performance of all three measures improves as the value of Rician $K$ increases. These results confirm that the proposed NLOS identification technique that is based on MIMO-OFDM is a good candidate for NLOS identification that can be employed in MIMO-OFDM systems. The proposed NLOS identification has applications in indoor and urban area localization. 


\section{Chapter 7}

\section{Conclusions and Open problems}

\subsection{Conclusions}

Chapter 2 conducts a thorough review on NLOS identification techniques proposed in the literature. NLOS identification methods have been categorized into cooperative and non cooperative. Each possesses different complexity and performance level. Cooperative techniques incorporate multiple transmitters or receivers to identify NLOS scenarios. However non cooperative techniques use only one receiver. The cooperative NLOS identification techniques performs properly when there are enough LOS measurements. In addition, the software complexity of these techniques is high. The non cooperative methods use different

measures statistics to identify NLOS scenarios. The measures include range statistics and channel characteristics. The range statistics based methods require a high SNR for TOA 
estimation and also require a long processing time to acquire statistics. Moreover, they fail to correctly identify NLOS when base nodes, the target node and the scatterers are all stationary. On contrary, the channel characteristics based, and hybrid methods offer a good trade-off between requirements and performance.

In Chapter 3, an approach is proposed to investigate the correlation across and within multiple users in multiuser MIMO narrow band wireless communication systems. The intra-user and inter-user correlations are theoretically derived and numerically evaluated. The impact of surface roughness on radio signal is also studied and simulation results show that higher roughness leads to lower correlation. In addition, topography, i.e., whether two users are in line with the significant scattering path, plays a main role in the shape of the cross-user correlation. For intra-user and inter-user cases, it is observed that LOS signals drastically boost the correlation and have a more flat trajectory in space. This property can be incorporated to discriminate LOS scenarios from NLOS scenarios.

In Chapter 4, a method based on the phase difference statistics across two antenna elements is proposed to identify NLOS scenarios in narrow band wireless systems. The proposed technique assumes uncorrelated phase across the two antenna elements. Simulations depict that this assumption is valid for elliptical distribution of scatterers as long as antenna element spacing exceeds one wavelength. However, for circular distribution of scatterers, antenna element spacing that ensures independency of the signal phase across antenna elements varies with the distance of transmitter and receiver: as the transmitter-receiver 
distance increases, higher antenna element spacing is required to ensure the independency of phases across two antenna elements. Thus, the proposed model performs better for elliptical models for which the antenna element spacing needs to be selected low. The elliptical model is a good model when the altitude of both the transmitter and receiver is low. This motivates the application of the proposed technique for near ground sensor networks in urban areas, such as those that could be installed on vehicles for traffic alert and collision avoidance. It is shown that smaller shadowing variance leads to a better NLOS identification performance. Typically, a variance of $4 \mathrm{~dB}$ leads to 0.7952 probability of identification while a variance of $8 \mathrm{~dB}$ leads to 0.7164 probability of identification. This technique can also be applied to systems with more than two antenna elements. Additional antennas increase the speed of data acquisition process and reduce the identification processing time.

Chapter 5 proposes to use space-frequency channel correlation based metrics for NLOS identification in frequency-selective and space-selective radio channels. Chapter 6 proposes to use space-time-frequency channel correlation based metrics for NLOS identification in time-varying, frequency-selective and space-selective radio channels. MIMO-OFDM signaling is used here as MIMO-OFDM technology is a good candidate for such channels. It is observed that in elliptical scattering microcell environments, the absolute value, the mean value and the standard deviation of SCD (based on space-frequency channel correlation) of NLOS are probably smaller than those of LOS. Simulations using WLAN $2 \times 2$ MIMO channel model shows that the technique using the mean and standard deviation has a higher identification performance than the technique using the absolute value of SCD. 
Among the four proposed mean and standard deviation metrics, identification using $\sigma$ and $\mu^{\text {abs }}$ offers the best performance, maintaining a high detection rate (around 98\%) with a low false alarm rate (around 4\%) for small values of Rician $K$ (e.g. in the order of one).

For large values of Rician $K$, the detection performance is poor and only $\mu^{\text {abs }}$ can offers a decent performance.

The use of metrics based on SCD assumes that the variation of spatial correlation across different multi-path components is minimal. This assumption does not hold in some channel models and in this case a group of metrics based on space-time-frequency channel correlation that are $\Lambda(N / 2), \Lambda(0)$ and $\Omega$ can be used. It is found that the performance of $\Omega$ beats that of $\Lambda(N / 2)$ and $\Lambda(0)$ when the space-time tap correlation is independent from tap to tap; the performance of $\Omega$ and $\Lambda(N / 2)$ is comparable and is better than $\Lambda(0)$ when the space-time tap correlation of neighboring taps is correlated. For both cases, the performance of all three measures increases as the value of Rician $K$ increases.

\subsection{Open problems}

\subsubsection{Practical issues in employing spatial correlation for NLOS identification}

In Chapter 3, the spatial channel correlation is derived based on the proposed multi-user MIMO channel model. This study can be extended to many other practical wireless environments. In addition, spatial channel correlation characterization of other MIMO channel 
models needs a further study to find the practical parameters used in identification process, such as the threshold separating correlation values of NLOS scenarios from those of LOS scenarios.

\subsubsection{In Chapter 4, what if $\operatorname{cov}\left(\Delta \phi_{1} \Delta \phi_{2}\right) \neq 0$}

In Chapter 4, a NLOS identification technique using phase difference across two antenna elements is proposed. In this technique, phases received at two antenna elements are assumed to be uncorrelated, i.e., $\operatorname{cov}\left(\Delta \phi_{1} \Delta \phi_{2}\right) \neq 0$ where $\Delta \phi_{1}$ and $\Delta \phi_{2}$ are shown in Figure 4.2. In some propagation environments, the uncorrelated phase assumption may not be applicable. Thus, alternative techniques need to be investigated for this situation.

\subsubsection{Space-time tap correlation model}

To the best knowledge of the author, in Section 5.3 the space tap correlation coefficient

$\chi_{l}^{p_{1}, q_{1} ; p_{2}, q_{2}}$ and elements of space-time tap correlation coefficient $\rho_{l}\left(p_{1}-p_{2}\right) \rho_{l}\left(q_{1}-q_{2}, \Delta t\right)$ in (6.16) are assumed to be uniformly distributed in a given range. Their realistic distributions could be further studied. These distributions would have an impact on the decision threshold and the performance of the proposed metrics. Therefore modeling of space-time tap correlation from a practical perspective would make the proposed metrics work better in real radio systems.

Closed forms of space-time correlation functions have been proposed in [56] and [60]. 
Those functions take into account various physical parameters such as the angle spreads at the transmitter and the receiver, the distance between transceivers, mean directions of the signal arrivals, array configurations, and Doppler spread. The proposed space-time correlation functions might be employed to study their distributions.

\subsubsection{Measures based on multi-dimensional space-time tap correlation}

In Section 6.2, all space-time-frequency correlation measures are based on the subcarrier correlation coefficient $\rho\left(p_{1}-p_{2}, q_{1}-q_{2}, n_{1}-n_{2}, \Delta t\right)$ defined in (6.6). There, a single value of $\rho\left(p_{1}-p_{2}, q_{1}-q_{2}, n_{1}-n_{2}, \Delta t\right)$ with transmit space separation $\left(p_{1}-p_{2}\right)$, receive space separation $\left(q_{1}-q_{2}\right)$ and time separation $\Delta t$ is used to identify NLOS scenarios. Note that multiple values of $\rho\left(p_{1}-p_{2}, q_{1}-q_{2}, n_{1}-n_{2}, \Delta t\right)$ could be obtained by varying $\left(p_{1}-p_{2}\right)$ or $\left(q_{1}-q_{2}\right)$ or $\Delta t$. Therefore, an extension on current work is to study what new measures can be formed based on a group of $\rho\left(p_{1}-p_{2}, q_{1}-q_{2}, n_{1}-n_{2}, \Delta t\right)$ with multiple sets of $\left(p_{1}-p_{2}\right),\left(q_{1}-q_{2}\right)$ and $\Delta t$. 


\section{References}

[1] I. Amundson and X. Koutsoukos, "A survey on localization for mobile wireless sensor networks," in 2nd International Workshop on Mobile Entity Localization and Tracking in GPS-less Environments (MELT), Sep 2009.

[2] S. Ingram, D. Harmer, and M. Quinlan, "Ultrawideband indoor positioning systems and their use in emergencies," in Position Location and Navigation Symposium, 2004. PLANS 2004, April 2004, pp. 706-715.

[3] Federal communications commission: Enhanced 911. [Online]. Available: http: //www.fcc.gov/pshs/services/911-services/enhanced911/

[4] W. Jones, "Keeping cars from crashing," Spectrum, IEEE, vol. 38, no. 9, pp. 40-45, Sep 2001.

[5] K. Amouris, "Position-based broadcast TDMA scheduling for mobile ad-hoc networks (MANETs) with advantaged nodes," in Military Communications Conference, 2005. MILCOM 2005. IEEE, Oct. 2005, pp. 252-257 Vol. 1.

[6] V. Sumathy, P. Narayanasmy, K. Baskaran, and T. Purusothaman, "GLS with secure 
routing in ad-hoc networks," in TENCON 2003. Conference on Convergent Technologies for Asia-Pacific Region, vol. 3, Oct. 2003, pp. 1072-1076 Vol.3.

[7] M. Rahman, M. Mambo, A. Inomata, and E. Okamoto, "An anonymous on-demand position-based routing in mobile ad hoc networks," in Applications and the Internet, 2006. SAINT 2006. International Symposium on, Jan. 2006, pp. 7 pp.-306.

[8] W. Foy, "Position-location solutions by taylor-series estimation," Aerospace and Electronic Systems, IEEE Transactions on, vol. AES-12, no. 2, pp. 187 -194, march 1976.

[9] X. Li, "A selective model to suppress NLOS signals in angle-of-arrival (AOA) location estimation," in the ninth IEEE international symposium on personal, indoor and mobile radio communications, Sep. 1998, pp. 461-465.

[10] F. Seco, A. Jimenez, C. Prieto, J. Roa, and K. Koutsou, "A survey of mathematical methods for indoor localization," in Intelligent Signal Processing, 2009. WISP 2009. IEEE International Symposium on, aug. 2009, pp. 9 -14.

[11] L. Konrad and W. Matt, "Motetrack: A robust, decentralized approach to rf-based location tracking," in International workshop on location- and context-awareness, vol. 3479, 2005, pp. 63-82.

[12] I. Guvenc, F. W. C. Chong, and H. Inamura, "Nlos identification and weighted leastsquares localization for uwb systems using multipath channel statistics," EURASIP Journal on Advances in Signal Processing, no. 36, Jan. 2008. 
[13] C. Falsi, D. Dardari, L. Mucchi, and M. Z. Win, "Time of arrival estimation for uwb localizer in realistic environment," EURASIP Journal on Applied Signal Processing, vol. 2006, 2006.

[14] M. Wylie and J. Holtzman, "The non-line of sight problem in mobile location estimation," in 5th IEEE international conf. on universal personal communications, Sep. 1996, pp. 827-831.

[15] A. Lakhzouri, R. H. E.S. Lohan, and M. Renfors, "Extended Kalman filter channel estimation for line-of-sight detection in WCDMA mobile positioning," EURASIP Journal on Applied Signal Processing, vol. 2003, no. 13, 2003.

[16] S. Yarkan and H. Arslan, "Identification of LOS in time-varying, frequency selective radio channels," EURASIP Journal on Wireless Communications and Networking, vol. 2008, pp. 1-14, Februrary 2008.

[17] B. Fleury, "First- and second-order characterization of direction dispersion and space selectivity in the radio channel," Information Theory, IEEE Transactions on, vol. 46, no. 6 , pp. $2027-2044$, sep 2000.

[18] L. Cong and W. Zhuang, "Nonline-of-sight error mitigation in mobile location," Wireless Communications, IEEE Transactions on, vol. 4, no. 2, pp. 560 - 573, march 2005.

[19] Y.-T. Chan, W.-Y. Tsui, H.-C. So, and P. chung Ching, "Time-of-arrival based localization under NLOS conditions," Vehicular Technology, IEEE Transactions on, vol. 55, no. 1 , pp. $17-24$, jan. 2006. 
[20] J. Borras, P. Hatrack, and N. Mandayam, "Decision theoretic framework for NLOS identification," in Vehicular Technology Conference, 1998. VTC 98. 48th IEEE, vol. 2, May 1998, pp. 1583-1587 vol.2.

[21] S. Venkatraman, J. J. Caffery, and H.-R. You, "Location using LOS range estimation in NLOS environments," in 55th IEEE Vehicular Technology Conference, 2002, pp. 856-860.

[22] S. Gezici, H. Kobayashi, and H. V. Poor, "Non-parametric non-line-of-sight identification," in Proceedings of the IEEE 58th Vehicular technology conference, vol. 4, Orlando, FL, USA, October 2003, pp. 2544-2548.

[23] N. Alsindi, M. Heidari, and K. Pahlavan, "Blockage identification in indoor uwb ranging using multi band ofdm signals," in Wireless Communications and Networking Conference, 2008. WCNC 2008. IEEE, 31 2008-April 3 2008, pp. 3231-3236.

[24] P.-C. Chen, "A non-line-of-sight error mitigation algorithm in location estimation," in Wireless Communications and Networking Conference, 1999. WCNC. 1999 IEEE, 1999, pp. 316-320 vol.1.

[25] S.-S. Woo, H.-R. You, and J.-S. Koh, “The NLOS mitigation technique for position location using IS-95 CDMA networks," in Vehicular Technology Conference, 2000. IEEE VTS-Fall VTC 2000. 52nd, vol. 6, 2000, pp. 2556-2560 vol.6.

[26] J. Schroeder, S. Galler, K. Kyamakya, and K. Jobmann, "NLOS detection algorithms 
for ultra-wideband localization," in 4th workshop on positioning, navigation and communication, Mar. 2007, pp. 159-166.

[27] L. Mak and T. Furukawa, "A time-of-arrival-based positioning technique with nonline-of-sight migtigation using low-frequency sound," Advanced Robotics, vol. 22, no. 5, pp. 507-526, 2008.

[28] J.S.Al-Jazzar and J. Caffery, "New algorithms for NLOS idetification," in IST mobile and wireless communication summit, Dresden, Germany, 2005.

[29] H. Bolcskei, "Fundamentals of wireless communications," March 2005, lecture hands out.

[30] F. Benedetto, G. Giunta, A. Toscano, and L. Vegni, "Dynamic LOS/NLOS statistical discrimination of wireless mobile channels," in Vehicular Technology Conference, 2007. VTC2007-Spring. IEEE 65th, April 2007, pp. 3071-3075.

[31] S. Yarkan and H. Arslan, "Identification of los and nlos for wireless transmission," in Cognitive Radio Oriented Wireless Networks and Communications, 2006. 1st International Conference on, 8-10 2006, pp. 1 -5.

[32] A. Molisch, D. Cassioli, C.-C. Chong, S. Emami, A. Fort, B. Kannan, J. Karedal, J. Kunisch, H. Schantz, K. Siwiak, and M. Win, "A comprehensive standardized model for ultrawideband propagation channels," Antennas and Propagation, IEEE Transactions on, vol. 54, no. 11, pp. 3151 -3166, nov. 2006. 
[33] A. Maali, H. Mimoun, G. Baudoin, and A. Ouldali, "A new low complexity NLOS identification approach based on UWB energy detection," in Radio and Wireless Symposium, 2009. RWS '09. IEEE, 18-22 2009, pp. 675-678.

[34] S. Venkatesh and R. Buehrer, "Non-line-of-sight identification in ultra-wideband systems based on received signal statistics," Microwaves, Antennas and Propagation, IET, vol. 1, no. 6, pp. 1120-1130, Dec. 2007.

[35] M. Heidari, N. Alsindi, and K. Pahlavan, "Udp identification and error mitigation in toa-based indoor localization systems using neural network architecture," Wireless Communications, IEEE Transactions on, vol. 8, no. 7, pp. 3597-3607, July 2009.

[36] L. Mucchi and P. Marcocci, "A new parameter for uwb indoor channel profile identification," Wireless Communications, IEEE Transactions on, vol. 8, no. 4, pp. 1597 -1602 , april 2009.

[37] A. Abbasi and M. Kahaei, "Improving source localization in los and nlos multipath environments for uwb signals," in Computer Conference, 2009. CSICC 2009. 14th International CSI, 20-21 2009, pp. 310-316.

[38] S. Marano, W. Gifford, H. Wymeersch, and M. Win, "Nonparametric obstruction detection for uwb localization," in Global Telecommunications Conference, 2009. GLOBECOM 2009. IEEE, nov. 2009, pp. 1 -6.

[39] W. Xu, Z. Wang, and S. Zekavat, "Non-line-of-sight identification in wireless local- 
ization via phase difference statistics across two antenna elements," IET Communications, accepted.

[40] K. Yu and Y. Guo, "Statistical NLOS identification based on AOA, TOA, and signal strength," Vehicular Technology, IEEE Transactions on, vol. 58, no. 1, pp. 274-286, Jan. 2009.

[41] N. Alsindi, C. Duan, J. Zhang, and T. Tsuboi, "Nlos channel identification and mitigation in ultra wideband toa-based wireless sensor networks," in Positioning, Navigation and Communication, 2009. WPNC 2009. 6th Workshop on, 19-19 2009, pp. 59 -66.

[42] I. E. Telatar, "Capacity of multi-antenna Gaussian channels," European Transactions on Telecommunications, vol. 10, no. 2, Nov. 1999.

[43] G. J. Foschini and M. J. Gans, "On limits of wireless communications in a fading environment when using multiple antennas," Wireless Personal Communications, vol. 6, no. $3,1998$.

[44] L. Zhong and D. N. C. Tse, "Diversity and multiplexing: a fundamental tradeoff in multiple-antenna channels," Information Theory, IEEE Transactions on, vol. 49, no. 5, May 2003.

[45] V. Tarokh, N. Seshadri, and A. R. Calderbank, "Space-time codes for high data rate wireless communication: performance criterion and code construction," Information Theory, IEEE Transactions on, vol. 44, no. 2, Mar. 1998. 
[46] P. W. Wolniansky, G. J. Foschini, G. D. Golden, and R. A. Valenzuela, "V-BLAST: an architecture for realizing very high data rates over the rich-scattering wireless channel," in Proc. URSI ISSSE, Pisa,Italy, Sep. 1998, pp. 295-300.

[47] W. C. Jakes, Microwave Mobile Communications. New York: Wiley, 1974, pp. $60-65$.

[48] D. Shiu, G. J. Foschini, M. J. Gans, and J. M. Kahn, "Fading correlation and its effect on the capacity of multielement antenna systems," Communications, IEEE Transactions on, vol. 48, no. 3, Mar. 2000.

[49] C. Chuah, D. N. C. Tse, J. M. Kahn, and R. A. Valenzuela, "Capacity scaling in MIMO wireless systems under correlated fading," Information Theory, IEEE Transactions on, vol. 48, no. 3, Mar. 2002.

[50] J. P. Kermoal, L. Schumacher, K. I. Pedersen, P. E. Mogensen, and F. Frederiksen, “A stochastic MIMO radio channel model with experimental validation," Selected Areas in Communications, IEEE Journal on, vol. 20, no. 6, Aug. 2001.

[51] A. M. Sayeed, "Deconstructing multiantenna fading channels," Signal Processing, IEEE Transactions on, vol. 50, no. 10, Oct. 2002.

[52] W. Weichselberger, M. Herdin, H. Ozcelik, and E. Bonek, "A stochastic MIMO channel model with joint correlation of both link ends," Wireless Communications, IEEE Transactions on, vol. 5, no. 1, Jan. 2006. 
[53] H. Ozcelik, N. Czink, and E. Bonek, "What makes a good MIMO channel model?" in IEEE Vehicular Technology Conference, May 2005, pp. 156-160.

[54] H. Tong and S. A. Zekavat, "On the suitable environments of the kronecker product form in MIMO channel modeling," in IEEE WCNC 2008, Las Vegas, Mar. 2008.

[55] R. B. Ertel, P. Cardieri, K. W. Sowerby, T. S. Rappaport, and J. H. Reed, "Overview of spatial channel models for antenna array communication systems," Personal Communications, IEEE, vol. 5, Feb. 1998.

[56] A. Abdi and M. Kaveh, "A space-time correlation model for multielement antenna systems in mobile fading channels," Selected Areas in Communications, IEEE Journal on, vol. 20, no. 3, Apr. 2002.

[57] M. Sharif and B. Hassibi, "On the capacity of MIMO broadcast channels with partial side information," Information Theory, IEEE Transactions on, vol. 51, no. 2, Feb. 2005.

[58] P. Beckmann and A. Spizzichino, The scattering of electromagnetic waves from rough surfaces. New York: Pergamon press, 1963.

[59] M. Steinbauer, A. Molisch, and E. Bonek, "The double-directional radio channel," Antennas and Propagation Magazines, IEEE, vol. 43, no. 4, Aug. 2001.

[60] G. Byers and F. Takawira, "Spatially and temporally correlated MIMO channels: 
Modeling and capacity analysis," Vehicular Technology, IEEE Transactions on, vol. 53, no. 3, May 2004.

[61] H. Tong and S. A. Zekavat, "Spatially correlated MIMO channel: Generation via virtual channel representation," Communications Letters, IEEE, vol. 10, no. 5, May 2006.

[62] T. Barton and D. Fuhrmann, "Covariance structures for multidimensional data," $M u$ tidimensional Systems and signal Processing, vol. 4, no. 2, Apr. 1993.

[63] W. Welford, "Optical estimation of statistics of surface roughness from light scattering measurements," Optical and Quantum Electronics, vol. 9, no. 4, Jul. 1977.

[64] D. Tse and P. Viswanath, Fundamentals of Wireless Communication. Cambridge University Press, 2005.

[65] "Digital mobile radio towards future generation systems," Cost 231 final report, Tech. Rep., 1999.

[66] W. Joseph, L. Martens, D. Plets, L. Verloock, E. Deventer, and H. Gauderis, "Extensive penetration loss measurements and models for different building types for DVB-H in the UHF band," Broadcasting, IEEE Transactions on, vol. 55, no. 2, pp. 213-222, June 2009.

[67] L. Thiele, M. Peter, and V. Jungnickel, "Statistics of the ricean K-factor at $5.2 \mathrm{Ghz}$ 
in an urban macro-cell scenario," in Personal, Indoor and Mobile Radio Communications, 2006 IEEE 17th International Symposium on, Sept. 2006, pp. 1-5.

[68] L. J. Greenstein, S. S. Ghassemzadeh, V. Erceg, and D. G. Michelson, "Ricean Kfactors in narrow-band fixed wireless channels: theory, experiments, and statistical models," Vehicular Technology, IEEE Transactions on, vol. 58, no. 8, pp. 4000-4012, Oct. 2009.

[69] P. Kyosti and et al. (2007, Sep) WINNER II channel models. [Online]. Available: http://www.ist-winner.org/WINNER2-Deliverables/D1.1.2v1.1.pdf

[70] S. M. Kay, Fundamentals of statistical signal processing, volume 2: detection theory. Prentice hall, February 1998.

[71] J. C. Liberti and T. S. Rappaport, "A geometrically based models for line of sight multipath radio channels," in IEEE vehicular technology conf., Apr. 1996, pp. 844848.

[72] L. Greenstein, D. Michelson, and V. Erceg, "Moment-method estimation of the ricean K-factor," Communications Letters, IEEE, vol. 3, no. 6, pp. 175-176, Jun 1999.

[73] A. Abdi, W. Lau, M.-S. Alouini, and M. Kaveh, "A new simple model for land mobile satellite channels: first- and second-order statistics," Wireless Communications, IEEE Transactions on, vol. 2, no. 3, pp. 519 - 528, May 2003.

[74] A. Peled and A. Ruiz, "Frequency domain data transmission using reduced compu- 
tational complexity algorithms," in Acoustics, Speech, and Signal Processing, IEEE International Conference on ICASSP '80., vol. 5, Apr. 1980, pp. 964 - 967.

[75] E. Jacobsen and et al, "IEEE P802.11 Wireless LANs," High Throughput Task Group, Tech. Rep. IEEE 802.11-03/940r4, May 2004.

[76] "IEEE standard for local and metropolitan area networks part 16: Air interface for broadband wireless access systems," IEEE Std 802.16-2009 (Revision of IEEE Std 802.16-2004), pp. C1 -2004, may. 2009.

[77] T. Lamahewa, R. Kennedy, T. Abhayapala, and T. Betlehem, "MIMO channel correlation in general scattering environments," in Communications Theory Workshop, 2006. Proceedings. 7th Australian, Feb. 2006, pp. 93 - 98.

[78] R. Ertel and J. Reed, "Angle and time of arrival statistics for circular and elliptical scattering models," Selected Areas in Communications, IEEE Journal on, vol. 17, no. 11, pp. $1829-1840$, nov 1999.

[79] S.-H. Kong, "TOA and AOD statistics for down link Gaussian scatterer distribution model," Wireless Communications, IEEE Transactions on, vol. 8, no. 5, pp. 2609 -2617 , may 2009.

[80] W. Xu and S. A. Zekavat, "High performance non-line-of-sight identification using MIMO-OFDM space frequency correlation statistics," in PIMRC, 2011. 


\section{Appendix A}

\section{Derivation of (3.33)}

Here, we intend to find:

$$
\mathrm{E}\left(\rho\left(\rho^{\prime}\right)^{*}\right)=\operatorname{Cov}\left(\rho, \rho^{\prime}\right)+\mathrm{E}(\rho) \mathrm{E}^{*}\left(\rho^{\prime}\right)
$$

Thus, we need to derive equations for $\operatorname{Cov}\left(\rho, \rho^{\prime}\right)$, the covariance of $\rho$ and $\rho^{\prime}$, and $\mathrm{E}(\rho) \mathrm{E}^{*}\left(\rho^{\prime}\right)$. Incorporating (3.30), we have:

$$
\begin{aligned}
& \operatorname{Cov}\left(\rho, \rho^{\prime}\right)=\frac{F\left(\theta_{1}, \theta_{2}\right) F\left(\theta_{1}, \theta_{2}^{\prime}\right)}{4 L^{2}} \int_{-L}^{L} \int_{-L}^{L} e^{i v_{x} x-i v_{x}^{\prime} x^{\prime}} \\
& \left(\chi_{2}\left(v_{z},-v_{z}^{\prime}\right)-\chi\left(v_{z}\right) \chi^{*}\left(v_{z}^{\prime}\right)\right) \mathrm{d} x \mathrm{~d} x^{\prime} \\
& \mathrm{E}(\rho) \mathrm{E}^{*}\left(\rho^{\prime}\right)=F\left(\theta_{1}, \theta_{2}\right) F\left(\theta_{1}, \theta_{2}^{\prime}\right) e^{-g_{1}} \operatorname{sinc}\left(v_{x} L\right) \operatorname{sinc}\left(v_{x}^{\prime} L\right)
\end{aligned}
$$


where $F\left(\theta_{1}, \theta_{2}\right)$ is defined in (3.32), $v_{z}$ and $v_{x}$ are defined in (3.31), $v_{x}^{\prime}=\left(\sin \theta_{1}-\sin \theta_{2}^{\prime}\right) 2 \pi / \lambda$, $v_{z}^{\prime}=-\left(\cos \theta_{1}+\cos \theta_{2}^{\prime}\right) 2 \pi / \lambda, \chi\left(v_{z}\right)=\exp \left(-\sigma^{2} v_{z}^{2} / 2\right)$ and $\chi_{2}\left(v_{z},-v_{z}^{\prime}\right)=\exp \left(-g_{1}+g_{2} e^{-\left(x-x^{\prime}\right)^{2} / T^{2}}\right), g_{1}$ and $g_{2}$ being defined under (3.33).

Note that the significant contributions to the integral merely come from the region near $\tau:=x-x^{\prime}=0$, i.e., $[-\delta, \delta]$. This is because for large $\tau, \chi_{2}\left(v_{z},-v_{z}^{\prime}\right)-\chi\left(v_{z}\right) \chi\left(v_{z}^{\prime}\right) \approx 0$. Then,

$$
\begin{aligned}
& \operatorname{Cov}\left(\rho, \rho^{\prime}\right) \approx=\frac{F\left(\theta_{1}, \theta_{2}\right) F\left(\theta_{1}, \theta_{2}^{\prime}\right)}{2 L} \operatorname{sinc}\left(\left(v_{x}-v_{x}^{\prime}\right) L\right) \\
& \int_{-\delta}^{\delta} e^{i v_{x} \tau}\left(\chi_{2}\left(v_{z},-v_{z}^{\prime}\right)-\chi\left(v_{z}\right) \chi^{*}\left(v_{z}^{\prime}\right)\right) \mathrm{d} \tau
\end{aligned}
$$

Expanding $\chi_{2}\left(v_{z},-v_{z}^{\prime}\right)$ in an exponential series, we have

$$
\chi_{2}\left(v_{z},-v_{z}^{\prime}\right)=e^{-g_{1}} \sum_{m=0}^{\infty} \frac{\left(g_{2} e^{-\tau^{2} / T^{2}}\right)^{m}}{m !}
$$

Substituting (A.5) in (A.4) we obtain

$$
\begin{aligned}
& \operatorname{Cov}\left(\rho, \rho^{\prime}\right) \approx \frac{F\left(\theta_{1}, \theta_{2}\right) F\left(\theta_{1}, \theta_{2}^{\prime}\right)}{2 L} \operatorname{sinc}\left(\left(v_{x}-v_{x}^{\prime}\right) L\right) e^{-g_{1}} \\
& \int_{-\infty}^{\infty} \cos \left(v_{x} \tau\right) \sum_{m=1}^{\infty} \frac{g_{2}^{m}}{m !} e^{-m \tau^{2} / T^{2}} \mathrm{~d} \tau
\end{aligned}
$$

where the integration limits $\pm \delta$ are replaced by $\pm \infty$; it is allowed since the integral receives 
significant contributions only from the region near $\tau=0$. Using the integral

$$
\int_{-\infty}^{\infty} e^{-a t^{2}} \cos (b t) \mathrm{d} t=\sqrt{\frac{\pi}{a}} e^{\frac{-b^{2}}{4 a}} \quad(a>0)
$$

we then find (3.33). 


\section{Appendix B}

\section{Derivation of (3.35)}

The series (3.33) converges too slowly to be of any practical use, so let's return to the integral (A.4). First, we note that for $g_{2} \gg 1, \chi\left(v_{z}\right) \chi\left(v_{z}^{\prime}\right) \approx 0$. Substituting in (A.4),

$$
\begin{aligned}
& \operatorname{Cov}\left(\rho, \rho^{\prime}\right) \approx \frac{F\left(\theta_{1}, \theta_{2}\right) F\left(\theta_{1}, \theta_{2}^{\prime}\right)}{L} \operatorname{sinc}\left(\left(v_{x}-v_{x}^{\prime}\right) L\right) e^{g_{2}-g_{1}} \\
& \int_{0}^{\delta} e^{-g_{2}\left(1-\exp \left(-\tau^{2} / T^{2}\right)\right)} \cos \left(v_{x} \tau\right) \mathrm{d} \tau
\end{aligned}
$$

It is easily verified that for $g_{2} \gg 1$, the only significant contribution to this integral comes from the region near $\tau=0$; we may therefore set $\exp \left(-\tau^{2} / T^{2}\right) \approx 1-\tau^{2} / T^{2}$ and replace the upper limit of integration by $\infty$, obtaining

$$
\begin{aligned}
& \operatorname{Cov}\left(\rho, \rho^{\prime}\right) \approx \frac{F\left(\theta_{1}, \theta_{2}\right) F\left(\theta_{1}, \theta_{2}^{\prime}\right)}{L} \operatorname{sinc}\left(\left(v_{x}-v_{x}^{\prime}\right) L\right) e^{g_{2}-g_{1}} \\
& \int_{0}^{\infty} e^{-g_{2} \tau^{2} / T^{2}} \cos v_{x} \tau \mathrm{d} \tau
\end{aligned}
$$


Applying (A.7), we then achieve (3.35). 


\section{Appendix C}

\section{Probability density function of $\Delta \phi_{1}$}

In Figure 4.2, without loss of generality, let $r_{\mathrm{LOS} 1}=A$. Let $R_{1}=\left|r_{1}\right|$, and the coordinates of $r_{1}$ is $\left(X_{1}, Y_{1}\right)$. Since $r_{\mathrm{DIF} 1}$ is zero mean complex Gaussian distributed with variance $2 \sigma^{2}$, $X_{1}$ has a normal distribution with mean $A$ and variance $\sigma^{2}$, and $Y_{1}$ has a normal distribution with mean 0 and variance $\sigma^{2}$.

$X_{1}$ and $Y_{1}$ can be expressed in terms of $R_{1}, \Delta \phi_{1}$,

$$
X_{1}=R_{1} \cos \Delta \phi_{1}, \quad Y_{1}=R_{1} \sin \Delta \phi_{1}
$$

Using a bivariate transformation of random variables, the joint PDF of $R_{1}$ and $\Delta \phi_{1}$ is

$$
f_{R_{1} \Delta \phi_{1}}\left(R_{1}, \Delta \phi_{1}\right)=f_{X_{1} Y_{1}}\left(R_{1} \cos \Delta \phi_{1}, R_{1} \sin \Delta \phi_{1}\right)\left|J\left(R_{1}, \Delta \phi_{1}\right)\right|
$$


where

$$
\begin{aligned}
J\left(R_{1}, \Delta \phi_{1}\right)= & \left|\begin{array}{cc}
\frac{\partial X_{1}}{\partial R_{1}} & \frac{\partial X_{1}}{\partial \Delta \phi_{1}} \\
\frac{\partial Y_{1}}{\partial R_{1}} & \frac{\partial Y_{1}}{\partial \Delta \phi_{1}}
\end{array}\right|=\left|\begin{array}{cc}
\cos \Delta \phi_{1} & -R_{1} \sin \Delta \phi_{1} \\
\sin \Delta \phi_{1} & R_{1} \cos \Delta \phi_{1}
\end{array}\right| \\
& =R_{1}\left(\cos ^{2} \Delta \phi_{1}+\sin ^{2} \Delta \phi_{1}\right)=R_{1}
\end{aligned}
$$

Now, the joint PDF of $X_{1}$ and $Y_{1}$ corresponds to:

$$
f_{X_{1} Y_{1}}\left(X_{1}, Y_{1}\right)=\frac{1}{2 \pi \sigma^{2}} \exp \left\{-\frac{\left(X_{1}-A\right)^{2}+Y_{1}^{2}}{2 \sigma^{2}}\right\}
$$

Hence, applying (C.3) and (C.4) into (C.2), $f_{R_{1} \Delta \phi_{1}}\left(R_{1}, \Delta \phi_{1}\right)$ is obtained. The marginal PDF of $\Delta \phi_{1}$ is calculated by intergrating over $R_{1}$ in (C.2),

$$
\begin{aligned}
& f_{\Delta \phi_{1}}\left(\Delta \phi_{1}\right)=\int_{0}^{\infty} f_{R_{1} \Delta \phi_{1}}\left(R_{1}, \Delta \phi_{1}\right) d R_{1} \\
& =\frac{1}{2 \pi \sigma^{2}} e^{-\frac{A^{2} \sin ^{2} \Delta \phi_{1}}{2 \sigma^{2}}}\left[\int_{0}^{\infty}\left(R_{1}-A \cos \Delta \phi_{1}\right) e^{-\frac{\left(R_{1}-A \cos \Delta \phi_{1}\right)^{2}}{2 \sigma^{2}}} d R_{1}+\right. \\
& \left.\int_{0}^{\infty} A \cos \Delta \phi_{1} e^{-\frac{\left(R_{1}-A \cos \Delta \phi_{1}\right)^{2}}{2 \sigma^{2}}} d R_{1}\right] \\
& =\frac{1}{2 \pi \sigma^{2}} e^{-\frac{A^{2} \sin ^{2} \Delta \phi_{1}}{2 \sigma^{2}}}\left[\sigma^{2} e^{-\frac{A^{2} \cos ^{2} \Delta \phi_{1}}{2 \sigma^{2}}}+A \cos \Delta \phi_{1} \sqrt{2 \pi} \sigma Q\left(-\frac{A \cos \Delta \phi_{1}}{\sigma}\right)\right] \\
& =\frac{\exp (-K)}{2 \pi}+\sqrt{\frac{K}{\pi}} \cos \Delta \phi_{1} \exp \left(-K \sin ^{2} \Delta \phi_{1}\right) \times Q\left(-\sqrt{2 K} \cos \Delta \phi_{1}\right), \\
& \phi_{1} \in[-\pi, \pi]
\end{aligned}
$$

where $K=\frac{A^{2}}{2 \sigma^{2}}$ and the second equality is created by using (C.2) and (C.4). 